\title{
DISCLAIMER
}

This report was prepared as an account of work sponsored by an agency of the United States Government. Neither the United Stales Government nor any agency thercof, nur any of theit employees. makes any warran:y, express or implied, or assumes any legal lisbiluty or responstbiljy for the accuracy. conpleteness. or usefulness of any infurmution, apparalus, product, or process disclosed, or represents that its use would not infringe prawtely uwned tights Reference herean to any specific commercial product, process, or vervice by trade name, trademark. manufacturer, or olherwise does nut necessarily conslitute or imply 1 ss endorsement, recommendation, or fitvoring hy the United States Government of any agency thereof The views and opinions of authors expressed herein do nut necessarily state or reflect those of the United States Government or any agency thereof.

UCRL --53871

DE89 004988

\section{Parametric Analysis of the Thermal Effects on the Divertor in Tokamaks During Plasma Disruptions}

\author{
Michael Lindsay Bruhn
}

(MS Thesis)

Manuscript date: April 1988

\section{LAWRENCE LIVERMORE NATIONAL LABORATORY University of California - Livermore, California - 94550}


Parametric Analysis of the Thermal Effects on the Divertor in Tokamaks During Plasma Disruptions

\author{
By \\ Michael Lindsay Bruhn \\ B.S. (United States Military Academy) 1981 \\ THESIS
}

Submitted in partial satisfaction of the requirements for the degree of MASTER OF SCIENCE

in

NUCLEAR ENGINEERING

in the

GRADUATE DIVISION

of the

UNIVERSITY' OF CALIFORNIA, BERKELEY

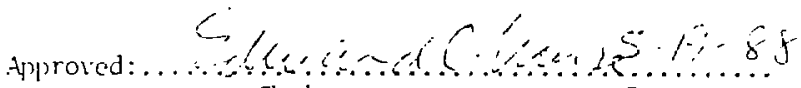

Chajrman Date

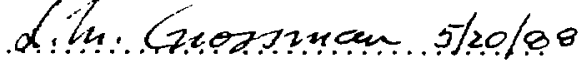

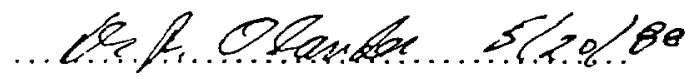




\section{ABSTRACT}

Plasma disruptions are an ever present danger to the plasma-facing components in today's tokamak fusion reactors. This threat results from our lack of understanding and limited ability to control this complex phenomenon. In particular, severe energy deposition occurs on the divertor component of the double-null configured tokamak reactor during such disruptions. A hybrid computational model developed to estimate and graphically illustrate global thermal effects of disruptions on the divertor plates is described in detail. This quasitwo-dimensional computer code, TADDPAK (Thermal Analysis Divertor during Disruptions PAcKage), is used to conduct parametric analysis for the TIBER Il Tokamak Engineering Test Rcactor Design. The dependence of these thermal effects on divertor material choice, disruption pulse length, disruption pulse shape, and the characteristic thickness of the plasma scrape-off layer is investigated for this reactor design. Results and conclusions from this analysis are presented. Improvements to this model and issues that require further investigation are discussed. Cursory analysis for ITER (International Thermonuclear Experimental Reactor) is also presented in the appendix.

APPROVED:

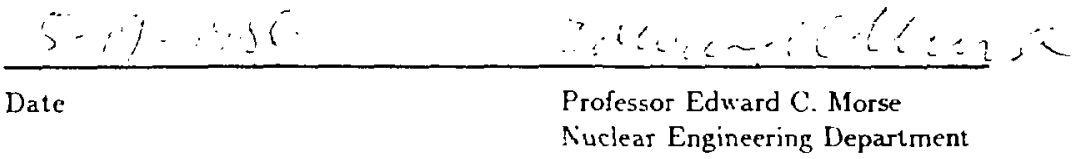




\section{ACKNOWLEDGEMENTS}

1 extend my sincere appreciation to I..J Perkins for his advice and help in the pursuit of this thesis. Without his guidance, this work would not have been possible.

Sincere thanks goes to Professor E. Morse for chairing my thesis committee and providing valuable guidance in writing this paper. I an also grateful to Professors L. Grossman and 1). Olander for serving on my committee.

I also thank W. Barr, B. Merrill, C. Croessmann, and J. Haines for their valuable discussions and help with the various aspects of this work.

I am indehted to Debbie Jalanivich and Donna Schreiber for their outstanding secretarial support while working at Lawrence Livermore National Laboratory.

Last, by not least, I extend my love and devolion to my wife Barbara. Only through her patience, reassurance, and understanding did I complete this achievernent. 


\section{BIOGRAPHY}

Captain Michael Lindsay Bruhn was born July 20, 1959, in Davenport, lowa. His parents are Mr. and Mrs. Daniel R. Bruhn of Bettendorf, lowa. He graduated from Bettendorf High School in 1977 and was accepted to the United States Military Academy that summer. He graduated from West Point and was commissioned a Second Lieutenant in the Army's Air Defense Branch in May 1981. He served with the 25th Infantry Division at Schofield Barracks, Hawaii, until February 1986. He was accepted into the Nuclear Engineering Department of University of California at Berkeley the fall semester of that year. He completed this thesis prior to graduating with a Masters of Science in May 1988. Following graduation the author will be assigned to the faculty at West Point where he will serve as an instructor in the Department of Physics for at least three years. He is married to the former Barbara Gorman Bradford, and they have one son, Robert. 


\section{Contents}

1 Introduction 1

1.1 Fusion Energy, Tokamaks, and Ivivertars . . . . . . . . . 1

1.2 Thesis Development. . . . . . . . . . . . . 5

1.3 Keperences for Chapter 1 ..............

2 Plasma Dismptions in Tokamaks 8

2.1 Review of Plasma Instability Therry . . . . . . . . . \&

2.2 Present Disruption Explanations . . . . . . . . . . 11

2.3 Jisruption Parameters ................. . . . . . . . .

2.4 J'asma Transport in the Serape-Off Layer . . . . . . . . . 16

2.5 I)ecription of Disruption (omputational Models (TSC, ISSTAR) 21

2.6 References for Chapter 2 . . . . . . . . . . . 25

3 Encrgy Deposition Theory 28

3.1 Thermal Response of Divertor Surface ........... 28

3.2 The lleat Gonduction Problem with Moving Bsundaries . . . . 31

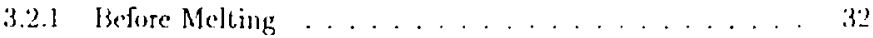


3.2.2 During and After Melting $\ldots \ldots \ldots \ldots \ldots$

3.2.3 Moving Boundary caused by Evaporation . . . . . . . 36

3.2 .4 Evaporation Model . . . . . . . . . . . . . 37

3.3 Numerical Methods . . . . . . . . . . . . . . . 39

3.4 References for Chapter $3 \ldots \ldots \ldots \ldots \ldots$. . . . . . 41

4 TADDPAK

4.1 Description . . . . . . . . . . . . . 42

4.2 Assumptions . . . . . . . . . . . . . 46

4.3 Material Properties . . . . . . . . . . . . . . 51

4.4 Graphical Output . . . . . . . . . . . . 53

4.5 References for Chapter $4 \ldots \ldots \ldots \ldots \ldots$

5 Parametric Analysis $\quad 57$

5.1 Choice of Parameters . . . . . . . . . . . . . . 57

5.2 Material Choice . . . . . . . . . . . . . . 59

5.3 Disruption Pulse Length . . . . . . . . . . . . . . . . 89

5.4 Disruption Pulse Shape . . . . . . . . . . . . . . . 99

5.5 Characteristic Radial Decay Length . . . . . . . . . . . . 110

5.6 References for Chapter $5 \ldots \ldots \ldots \ldots \ldots$

6 Conclusions and Recommendations for Further Investigation 122

6.1 Conclusions . . . . . . . . . . . . . . . . 122

6.2 Future Work . . . . . . . . . . . . . . . . 125 
A 'TADDPAK User Information

B Preliminary Results for US I'THR 


\section{List of Figures}

1.1 Tokamak reactor (from reference $[1.1]$ ) . . . . . . . 2

1.2 Double-null divertor configuration for TIBER II (from reference

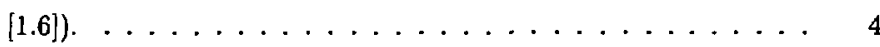

2.1 Magnetic topography of a poloidal cross section of the TIBER II design. . . . . . . . . . . . . .

2.2 Disruption power flow as a function of time generated by DSTAR for a $0.1-\mathrm{ms}$ thermal quench of $105 \mathrm{MJ}$. . . . . . . .

3.1 Schematic representation of plasma disruption-material interaction (from reference $[3.1]) \ldots \ldots \ldots \ldots \ldots$

3.2 Schematic representation of the solid, liquid, and vapor phases (from Reference $[3.1]) . \ldots \ldots \ldots \ldots \ldots$

4.1 TADDPAK flow chart structure. . . . . . . . . 43

4.2 Example of TADDPAK graphical output. . . . . . . 54

5.1 Disruption affects $(0.1 \mathrm{~ms})$ on inner divertor plate for graphite divertor material showing distribution of power/energy densities. 
5.2 Disruption effects $(0.1 \mathrm{~ms})$ on lower divertor plate for graphite divertor material showing distribution of power/energy densities.

5.3 Disruption effects $(0.1 \mathrm{~ms})$ on outer divertor plate for graphite divertor material showing distribution of power/energy densities.

5.4 Disruption effects $(0.1 \mathrm{~ms})$ on inner divertor plate for grap'ite divertor material showing distribuiion of surface temperature.

5.5 Disruption elfects $(0.1 \mathrm{~ms})$ on lower divertor plate for graphite divertor material showing distribution of surface temperature. .

5.6 Disruption effects $(0.1 \mathrm{~ms})$ on outer divertor plate for graplite divertur material showing distribution of surface temperature.

5.7 Disruption effects $(0.1 \mathrm{~ms})$ on inner divertor plate for graphite divertur material showing distribution of vaporization rate and ablaticn depth. . . . . . . . . . . . . .

5.8 Disruption effects $(0.1 \mathrm{~ms})$ on lower divertor plate for graphite divertor material showing distribution of vaporization rate and alblation depth.

5.9 Disruption effects $(0.1 \mathrm{~ms})$ on vuter diverlor plate for graphite divertor material showing distribution of vaporization rate and ablation depth.

5.10 1)isruption effects (0.1 ms) on inner divertor plate for tungsten divertur material showing dist ribution of surface temperalure. 
5.11 Disruption effects $(0.1 \mathrm{~ms})$ on lower divertor plate for tungsten divertor material showing distribution of surface temperature. .

5.12 Disruption effects $(0.1 \mathrm{~ms})$ on outer divertor plate for tungsten divertor material showing distribution of surface temperature. .

5.13 Disruption effects $(0.1 \mathrm{~ms})$ on inner divertor plate for tungsten divertor material showing distribution of vaporization rate and

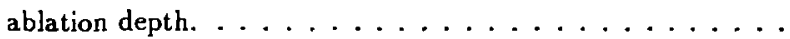

5.14 Disruption effects $(0.1 \mathrm{~ms})$ on lower divertor plate for tungsten divertor material showing distribution of vaporization rate and ablation depth. . . . . . . . . . . .

5.15 Disruption effects $(0.1 \mathrm{~ms})$ on outer divertor plate for tungsten divertor material showing distribution of vaporization rate and

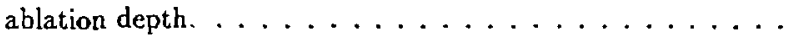

5.16 Disruption effects $(0.1 \mathrm{~ms})$ on inner divertor plate for beryllium divertor material showing distribution of surface temperature.

5.17 Disruption effects $(0.1 \mathrm{~ms})$ on lower divertor plate for beryllium divertor material showing distribution of surface temperzture. .

5.18 Disruption effects $(0.1 \mathrm{~ms})$ on outer divertor plate for beryllium divertor materjal showing distribution of surface temperature. .

5.19 Disruption effects $(0.1 \mathrm{~ms})$ on inner divertor plate for beryllium divertor material showing distribution of vaporization rate and

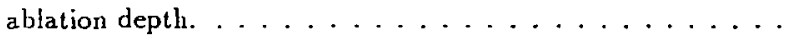


5.20 Disruption effects $(0.1 \mathrm{~ms})$ on lower divertor plate for beryllium divertor material showing distribution of vaporization rate and ablation denth. . . . . . . . . . .

$5 . \bar{i}$ Disruption effects $(0.1 \mathrm{~ms})$ on outer divertor plate for beryllium divertor material showing distribution of vaporization rate and ablation depth. . . . . . . . . . . .

5.22 Niaximum temperature on each graphite divertor plate as a function of disruption pulse length. $\ldots \ldots \ldots \ldots \ldots$

5.23 Maximum vaporization rate on each gr iphite divertor piate as a function of disruption pulse length. . . . . . . . . .

5.24 Maximum vaporization depth on each graphite divertor plate as a function of disruption pulse length. . . . . . . . . . .

5.25 Vaporization peaking factors on each graphite divertor plate as a function of disruption puise length. . . . . . . . . . .

5.26 Total volume ablated from each graphite divertor plate as a function of disruption pulse length for one hemisphere of the machine. 98

5.27 Disruption effects of $0.1 \mathrm{~ms}$ square pulse on inner divertor plate for graphite divertor material showing distribution of power and encrgy densities. . . . . . . . . . . . . 100

5.28 Disruption effects of $0.1 \mathrm{~ms}$ square pulse on lower divertor plate for graphite divertor material showing distribution of power and energy densities. 
5.29 Disruption effects of $0.1 \mathrm{~ms}$ square pulse on outer divertor plate for graphite divertor material showing distribution of power and energy densities. . . . . . . . . . . . . . . 102

5.30 Disruption effects of $0.1 \mathrm{~ms}$ square pulse on inner divertor plate for graphite divertor material showing distribution of surface temperature

5.31 Disruption effects of $0.1 \mathrm{mis}$ square pulse on lower divertor plate for graphite divertor material showing distribution of surface temperature

5.32 Disruption effects of 0.1 ms square pulse on outer divertor plate for graphite divertor material showing distribution of surface ternperature.

5.33 Disruption effects of $0.1 \mathrm{~ms}$ square pulse on innei divertor plate for graphite divertor material showing distribution of vaporization rate and ablation depth.

5.34 Disruption effects of $0.1 \mathrm{~ms}$ square pulse on lower divertor plate for graphite divertor material showing distribution of vaporization rate and ablation depth. . . . . . . . . . 108

5.35 Dis ruption effects of $0.1 \mathrm{~ms}$ square pulse on outer divertor plate for graphite divertor material showing distribution of vaporization rate and ablation depth. 
5.36 Maximum disruption power density on each graphite divertor plate as a function of characteristic radial decay length. . . . . 112

5.37 Total energy deposited on each graphite divertor plate as a function of characteristic radial decay length. . . . . . . . 114

5.38 Maximum surface temperature on each graphite divertor plate as a function of characteristic radial decay length. . . . . 116

5.39 Maximum vaporization rate on each graphite divertor plate as a function of characteristic radial decay length. . . . . . 117

5.40 Maximun vaporization depth on each graphite divertor plate as * function of characteristic radial decay length. . . . . . 118

5.41 Total volume ablated on each graphite divertor plate as a function of characteristic radial decay length. . . . . . . . . 120

B.1 Disruption conditions on the inner divertor plate for graphite divertor material showing distributions of power/energy densities, surface temperature, and vaporization rate/ablation depth. . . . 131

B.2 Disruption conditions on the lower divertor plate for graphite divertor inatcrial showing distributions of power/energy densities, surface temperature, and vaporization rate/ablation depth. . . 132

B.3 Disruption conditions on the outer divertor plate for graphite divertor matcrial showing distributions of power/energy densities, surface temperature, and vaporization rate/ablation depth. . . 133 
B.4 Disruption conditions on the inner divertor plate for tungsten divertor material showing distributions of power/energy densities, surface temperature, and vaporization rate/ablation iepth. . . 134

B.5 Disruption conditions on the lower divertor plate for tungsten divertor material showing distributions of power/energy densities, surface temperature, and vaporization rate/ablation depth. . . 135

B.6 Disruption conditions on the outer divertor plate for tungsten divertor material showing distributions of power/energy densities, surface temperature, and vaporization rate/ablation depth. . . 136 


\section{List of Tables}

2.1 Assumed disruption parameters for TIBER 11 design. . . . . 15

4.1 Characteristic diffusion times of the magnetic field . . . . . . 48 48

4.2 Thermophysical properties and function constants . . . . . 52

4.3 Constants for saturation pressure function Equation $4.5 \ldots 53$

5.1 Summary of disruption power and energy densities on graphite divertor plates subjected to 0.1 ms disruption. . . . . . . 65

5.2 Summary of ablation effects on graphite divertor plates subjected to $0.1-$ ms disruption. . . . . . . . . . . . . 73

5.3 Comparison of ablation effects on divertor plates of graphite, tungsten, and beryllium subjected to 0.$] \mathrm{ms}$ disruption. . . . 80

5.4 Disruption erosion lifetime parameters for graphite, tungsten, and beryllium subjected to 0.1 ms disruption. . . . . . . . 89

5.5 Comparison of ablation effects on graphite divertor plates due to the DSTAR and square pulse shapes for a 0.1 ns disruption. . . 111 
6.1 Summary of ablation effects on divertor plates of graphite, tungsten, and beryllium subjected to 0.1 ms disruption. . . . . . . 126

A.1 Descriptions of files found in PAK.LB . . . . . . . . . 129 


\section{Chapter 1}

\section{Introduction}

\subsection{Fusion Energy, Tokamaks, and Divertors}

The attempt to harness the energy from a nuclear fusion reaction similar to the reaction present on our sun, and provide from it commercial electrical power, is a continuing quest of today's nuclear scientists and engineers. During the past forty years of this effort to create, in essence, a s.nall star on earth, fusion energy research has branched into the two general categories of magnetic and inertial confinement. The first approach relies on various geometries of strong magnetic fields to confine an ionized gas of hydrogen isotopes or plasma at the densities and temperatures necessary for the fusion reaction to occur. The second approach seeks to ignite a frozen hydrogen fuel pellet with multiple surrounding laser or particle beams, to achieve the necessary densities and temperatures from the resulting pellet implosion.

The magnetic confinement category of reactor design has had several machine configurations investigated and developed during the past three decades. 


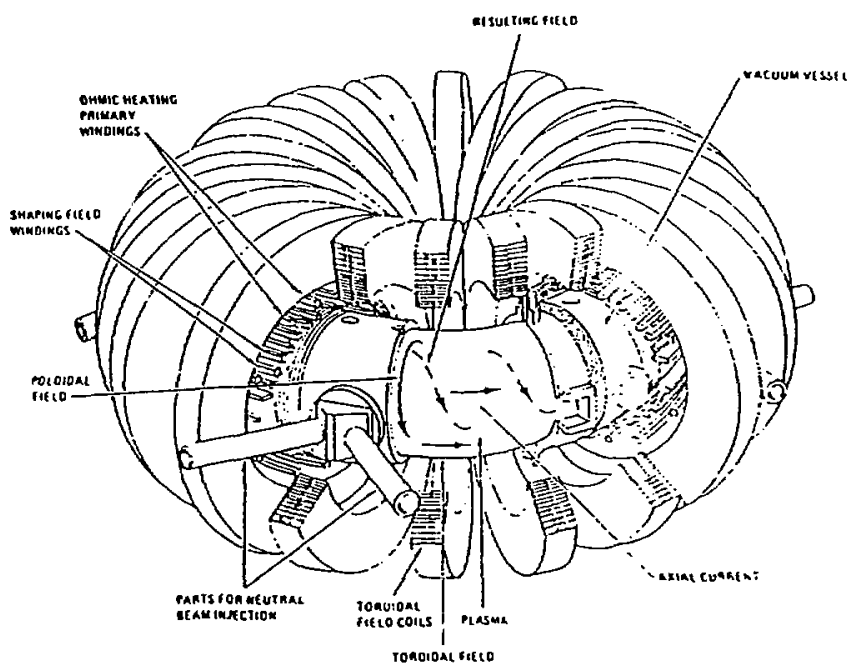

Figure 1.1: Tokamak reactor (from reference [1.1]).

The machine designs include the tandem mirror, reverse field pinch, compact torus, and tokamak. By far the most international investigation of and progress toward developing a functional magnetic confinement reactor has occurred with the tokamak design. This torus-shaped configuration, illustrated in Figure 1.1, is the most advanced reactor design. Despite this claim, many physics and engineering problems have hindered the development of a commercial reactor. One of the most significant problems faced by tokamak designers is the phenomenon of plasma disruptions. A plasma disruption is a rapid loss of plasma confine- 
ment that can occur anytime during the normal operation of a tokamak reactor. Present knowledge of plasma disruptions divide thern into two phases. The first phase is the thermal quench phase, during which a majority of the plasma's particle kinetic energy is lost to the plasma-facing components. The second phase is the current decay phase, during which the plasma's stored magnetic energy is lost through inductively created bulk material forces. The actual physical processes behind these phases will be discussed in more detail in Chapter 2. The release of the particle kinetic energy during the thermal quench phase poses lize most significant erosion threat to the plasma-facing components. These components include first-wall armor inaterials, neutral beam dumps, $r$ antennas, diagnostic equipment, and limiters or divertors depending on the specific machine design.

One such tokamak ciesign is the double-null divertor configuration illustrated in Figure 1.2. This magnetic configuration has been investigated by several continuing reactor design studies. Examples of these studies include INTOR (International Tokamak Reactor) [1.2] and NET (Next European Torus) [1.3]. This configuration is also intended for use on future devices such as CIT (Compact lgnition Tokamak) [1.4] and ITER (International Thermonuclear Experimental Reactor) [1.5]. One of the most recent design studies for the double-null divertor configuration is TlBER Il (Tokamak Ignition/Burn Engineering Reactor Two) [1.6]. This steady-state tokamak design has a 3 -meter major radius, employs non-inductive current drive, and utilizes a symmetric double-null divertor of 

(1) LONTR SUPPoAT SHIELO
(D) OH SUPPORT SJaUc tuRE

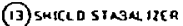
(2) UPPER SUPPOAT SHILLD
(9) radoun riclo cons
(14) Be ameC I TCST nOOVRE
(3) IKBOARO 5 HIECLD
(9) Pr sumory stauc tuac
(15) LOMTA DIVERTOA
(4) OUiBOARD SMIELD
(10) ChYOCEKIC SUPPORT PLATE
(16) Upeer diveator
(5) Toroloul rielo cons
(11) CaTOCENIC SUPPORT STRUTS

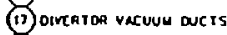
(5) onmic neat inc colcs
(12) SHICLO SUPPOAT PEOCSTML.
(18) outen racuene wate
(19) uncure dout

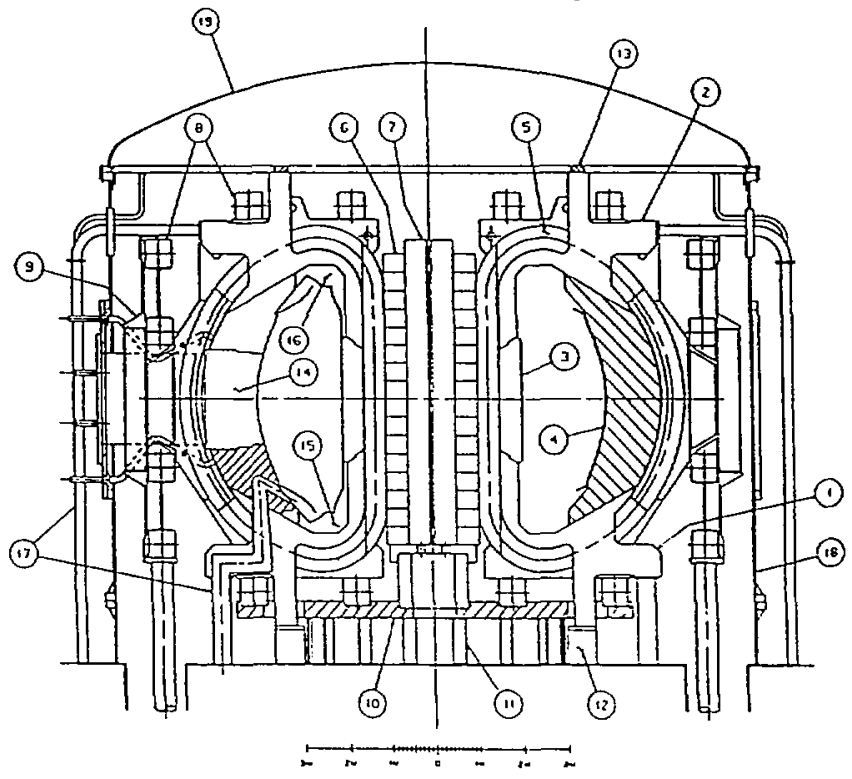

Figure 1.2: Double-null divertor configuration for TIBER II (from reference [1.6]). 
approximately 80 square meters. Since this divertor comprises approximately $35 \%$ of the reactor's total surface area facing the plasma, the thermal effects of disruptions on the divertor are a highly significant design issue.

\subsection{Thesis Development}

The purpose of this thesis is to develop a quasi-two-dimensional computer code package to theoretically model the above-mentioned thermal effects and to conduct perametric analysis with this code, specifically for the TIBER II reactor design. TADDPAK (Thermal Analysis Divertor during Disruptions PAcKage) is the result of this effort. The motivation behind this investigation is threefold. First, TADDPAK was designed to provide a more detailed analysis of total divertor surface erosion during disruptions. Past analysis has focused mainly on a purely one-dimensional treatment of this problem. TADDPAK also uses a one-dimensional model for the heat conduction/vaporization problem. However, TADDPAK applies this one-dimensional model to a partitioned two-dimensional cross section of the divertor. Second, TADDPAK was developed to help meet one of the many design needs of future tokamak reactors. This code package is generic in the sense that it can be easily adapted to most divertor/tokamak double-null configurations, and as a public domain file, can be obtained b; ary. one as outlined in this document's appendix. Third, TADDPAK was created to minimize the computer time requirement for parametric analysis as compared to already existing and more complex computer codes. For example, DSTAR [1.7], 
written by $\mathrm{B}$. Merrill and $\mathrm{S}$. Jardin, is a complex plasma simulation code which quantifies the surface erosion and induced forces that can occur during a major plasma disruption. Besides being originally designed for a limiter configuration, this code requires between 30 and 40 minutes of computer time per disruption calculation. Although DSTAR calculates a wealth of information relevant to the overall reactor design, detailed parametric analysis of one specific component is more easily accomplished with a computer time efficient and problem specific code. The relatively short computational time required by TADDPAK and its specific computational analysis is based on several simplifying assumptions, outlined in Chapter 4. TADDPAK is one of the many small but important design tools able to contribute to the future development of commercial fusion energy.

A more detailed description of plasma disruptions in tokamak reactors and the resulting energy deposition on the divertor is given in Chapters 2 and 3. Chapter 4 describes the structure and development of TADDPAK in detail. One of TADDPAK's valuable features is its three-dimensional graphic presentation of computational results. These results of parametric analysis for the TIBER II reactor design are presented in Chapter 5 . The interpretation of and conclusions from these results for the TIBER II design, as well as implications for the doublenull divertor tokamak design in general, are given in Chapter 6. 


\subsection{References for Chapter 1}

1.1 J.M. Rawls et al., "Status of Tokamak Research," DOE/ER-0034 (1979), Fig. 1-1.

1.2 "US Contribution to the Phase 2A, Part 2, INTOR Workshop, 198385"(July 1985).

1.3 R. Albanese, M. Biggio, et al., "NET Status Report," Commission of the European Communities Directorate General XII-Fusion Programme, Brussels, West Germany (1985) 333.

1.4 R. Parker et al.,CIT Physics Design Description, Princeton Plasma Physics Laboratory (AE-880112-PPL-01) (January 1988).

1.5 L.J. Perkins, Lawrence Livermore National Laboratory, private communication, 1987.

1.6 J.D. Lee, III, Ed., "TIBER II/ETR Final Design Report," Lawrence Livermore National Laboratory, UCID-21150, (September 1987).

1.7 B.J. Merrill and S.C. Jardin, "User's Manual for DSTAR: A Comprehensive Tokamak Disruption Code," Idaho National Engineering Laboratory, LGG-EP-7394 (October 14, 1986). 


\section{Chapter 2}

\section{Plasma Disruptions in Tokamaks}

\subsection{Review of Plasma Instability Theory}

Confined tokamak plasmas exhibit several different types of large-scale instabilities. The rnost widely observed exarnples of these unstable phenomena include Mirnov oscillations, sawtooth oscillations, and disruptive instabilities. Many aspects of this unstable behavior can be predicted and categorized by magnetohydrodynamic (MHD) theory [2.1]. This theory combines certain fluid equations and Maxwell's electrodynamics equations into a relatively simple mathematical model. The model describes the interaction between the plasma and the surrounding magrietic forces. Depending on th a problem's temperature regime, the plasma is treated as a fluid with either infinite or finite electrical conductivity. According to this theory, MHD instabjlitics include interchange, kink, and tearing modes. Pressure gradients and magnetic field curvature drive the interchange modes, while electrical currents parallel to the magnetic field drive 
the kink and tearing modes. The interchange mode, generally a tolerable and localized instability, causes discrete volumes of the plasma to change locations with each other. The difference between the kink and tearing modes is distinguished by the magnitude of the plasma's electrical conductivity. Kink modes occur in an ideal or perfectly conducting plasma while tearing modes occur in a resistive plasma. As the name suggests, kink modes actually kink the plasma column through a torque arising from radial variations in the toroidal current density [2.2]. Tearing modes go a step further. These modes kink the plasma column to the point of actually tearing the magnetic field lines and creating localized magnetic islands within the plasma. Each magnetic island created is an individual set of nested flux surfaces centered on its own magnetic axis. All of these modes can be further described as either internal or external, and characterized mathematically by the plasma displacement function $\delta$ where

$$
\delta \sim e^{i m \theta+i n \phi} .
$$

Here $\theta$ and $\phi$ are the poloidal and torodial angles of the torus geometry, respectively. The mode numbers $m$ and $n$ are integers corresponding to the shape of the plasma's deformation and should not be confused with the different types of instability modes.

These MHD instabilities provide a basis to characterize what have been previously distinguished as large-scale instabilities in tokamaks. The safety factor $q$ is also used when discussing plasma instabilities. The safety factor is 
defined by

$$
q=d \psi_{t} / d \psi_{p}
$$

where $d \psi_{t}$ and $d \psi_{p}$ are the rates of change of the magnetic flux passing through a given toroidal and poloidal surface, respectively. The safety factor represents the amount of twist in the helical magnetic field lines of a torus and can be approximated by

$$
q \approx r B_{t} / R_{o} B_{p}
$$

where $B_{\iota}$ and $B_{p}$ are the toroidal and poloidal magnetic fields, respectively; $r$ is the plasma minor radius; and $R_{o}$ is the plasma major radius. For example, Mirnov oscillations are an external instability that occur at the plasma edge. During normal tokamak operation, these small oscillating helical perturbations in the poloidal magnetic field can be detected by magnetic surface probes [2.3]. This instability is currently believed to result from nonlinearly saturated magnetic islands produced by resistive tearing modes [2.1]. In contrast, sawtooth oscillations are internal magnetic perturbations within normal tokamak plasmas. Von Goeler et al. [2.4] were among the first to observe this instability in 1974 through the detection of soft X-ray cmissions from the plasma core. Although Mirnov and sawtooth oscillations are interesting phenomena and occur during normal tokamak operation, they apparently pose little or no threat to plasma confin 'ment or structural integrity. However, the phenomenon defined under the generic term disruptive instabilities poses one of the most significant threats to tokamak operation. 
The major disruption is considered to be the most serious type of disruptive instability. Major disruptions occur abruptly with a rapid loss of plasma current and confinement. They are characterized by a negative voltage spike, a positive current spike, fast inward motion of an expanding plasma column, and intense outward particle fluxes. These particle fluxes, in turn, cause severe transient energy deposition and significant vaporization of reactor components. Because of this potentially damaging effect of major disruptions, they are the disruptive instability of interest in this thesis. The mathernatical modeling of major disruptions provides one form of input data for the subsequent analysis of thermal effects on the divertor of a specific tokamak design. Hence, the following general explanation of major disruptions is relevant to this thesis. From this point forward, any discussion of distuptions will be referring only to major disruptions.

\subsection{Present Disruption Explanations}

Since the discovery of disruptive instabilities in 1963 by Gorbunov and Razumova [2.5], scientists have launched a theoretical and expcrimental effort to explain and control this potentially deleterious phenomenon. Three different theoretical explanations for disruptions have emerged based on MHD theory. The common view of these explanations is that the $2 / 1(\mathrm{~m}=2 / \mathrm{n}=1)$ tearing Inode and its resulting magnetic jslands serve as a catalyst in the development of disruptions [2.6-2.10]. One theory, proposed by Sykes and Wesson [2.10], 
views the disruption as the interaction of a growing $2 / 1$ magnetic island and the cold edge of the plasma. The interaction results in the rapid cooling of the bulk plasma. This theory is supported by experiments conducted by Toi [2.11] for relatively low- $q$ discharges. Another theory, first proposed by Turner and Wesson [2.12] and later reinforced by S. Tsuji et al. [2.13], explains disruptions in terms of a nonlinear interaction between the $m=1$ and $m=2$ modes occurring through the mediator of the current profile. The $1 / 1$ tearing mode appears to trigger disruptions in the presence of the $2 / 1$ magnetic island in both highdensity and low- $q$ djscharges. This interaction, resulting in catastrophic energy loss, can be inferred from several past tokamak experiments [2.13-2.18].

The third explanation, given by Waddell et al. [2.19], provides important insight to the theoretical understanding of disruptions and appears to be the more widely accepted theory. The theory proposes that certain current profiles induce growing $2 / 1$ and $3 / 2$ tearing modes. These modes, along $w i t h$ other excited helicities, interact in a violent and nonlinear manner. This interaction results in the overlapping of magnetic islands and the subsequent degeneration of the magnetic field. Stcchastic field lines then cause enhanced electron transport, which leads to the rapid cooling of the plasma and the total loss of confinement. Several past experiments support this interpretation of disruptions [2.20-2.22]. The computer code discussed in Section 2.5 assumes this theory when modeling disruptions.

At present, there is not enough experimental data to conduct a detailed 
comparison between these theories and experiments for every aspect of this enigmatic phenomenon. Each theory discussed can only qualitatively describe many of the global characteristics typically observed during disruptions. The adoption of any particular theory is, at best, a scientific estimate for attempting to model a complex event. A more detailed understanding will only be possible through continued systematic experimentation and analysis. Hence, the process of disruption modeling will evolve as future experiments uncover the numerous intricacies of this phenomenon. The most recent effort to compile and analyze such data occurred during the July 1987 INTOR Disruption Workshop in Vienna [2.23]. A group of scientists, representing most of the current major tokamak experiments, analyzed and compared their disruption data. Their conclusions are used in the next section to decide upon applicable parameters for this thesis.

\subsection{Disruption Parameters}

The first step in conducting any type of detailed analysis on the effects of disruptions, based on the present limited experimental data, is to make judicious assumptions on their key characteristics. Since the highest power densities at the divertor occur during the thermal quench phase of a disruption, this phase is the focus of the following research. Therefore, the parameters of importance for this study include: 
- The total amount of the plasma's stored thermal energy available for deposition at the divertor surface.

- The percentage of the thermal energy that arrives at each divertor plate as radiation.

- The percentage of the thermal energy that arrives at each divertor plate as an intense particle flux.

- The distribution of this energy along the divertor plates.

- The deposition time for this energy at the divertor.

The thermal energy per unit volume of a plasma consisting of a group of particles, each with three deg،ees of freedom, is defined by

$$
E=\frac{3}{2} \sum_{i=1}^{n} n_{i} k T_{i},
$$

where $k$ is Boltzmann's constant and $T_{i}$ is the temperature of each particle. 'The pressure exerted by these particles is simply

$$
P=\sum_{i=1}^{n} n_{i} k T_{i}
$$

where $n_{i}$ is the average density per particle specjes. For tokamaks, the ratio of the average plasma pressure to the axjal toroidal magnetic pressure is normally expressed as the average total beta or

$$
<\beta_{T}>=\frac{\langle P}{B^{2} / 2 \mu_{0}}
$$


Table 2.1: Assumed disruption parameters for TIBER II design.

\begin{tabular}{||r|r|}
\hline Parameter & Value \\
\hline Average Total Beta (\%) & 6.0 \\
Magnetic Field (T) & 5.55 \\
Plasma Volume (m $\left.{ }^{3}\right)$ & 96.1 \\
Store Thermal Energy (MJ) & 105 \\
Radiated Energy (30\%) (MJ) & 31.5 \\
Particle Flux Energy (70\%) (MJ) & 73.5 \\
Inner/Outer SOL (Scrape-Off Layer) Energy Partition (ratio) & $1: 2$ \\
Energy Deposition Tirne (ms) & 0.1 \\
Characteristic Decay Length of SOL (cm) & 3.0 \\
\hline
\end{tabular}

where $B$ is the magnetic field on axis and $\mu_{o}$ is the permeability of free space. If Equations 2.4-2.6 are combined, a more useful equation for the stored thermal energy can be derived:

$$
E=\frac{3}{2}<\beta_{T}>\frac{B^{2}}{2 \mu_{\circ}} V_{P},
$$

where $V_{P}$ is the plasma volume. Using the TIBER II design values listed in Table 2.1, the stored thermal energy is approximately $105 \mathrm{MJ}$.

The fraction of thermal energy that goes to the divertor as radiation depends very much on the machine design. The device specifications regulate the level of plasma impurities, which, in turn, can determine the amount of radiation emitted isotropically from the disrupting plasma. This value has been assumed t. . as high as $50 \%$ in NET $[2.24\}$ and as low as $30 \%$ in INTOR $\{2.25\}$. For I IBER II, INTOR's fraction of $30 \%$ is the adopted value, and the corresponding energy is 31.5 MJ. Assuming isotropic emission, the percentage of the first-wall surface area comprised by the divertor, multiplied by this amount of radiated 
energy, then determines the frartion of the stored thermal energy impinging on the component as radiation. Based on this assumption for radiation, approximately $70 \%$ of the stored thermal energy leaves the edge of the plasma in the form of an intense particle flux. The assumed physical mechanism by which the particle flux reaches and is distributed over the divertor plates for the double-null configuration is discussed in detail in Section 2.4.

The deposition time of the thermal energy during a disruption is a controversial issue. Past design studies have assumed values on the order of 1-20 ms [2.24-2.26]. However, recent experimental data indicate a much shorter time scale and, hence, considerably higher power densities. For example, in Dlll-D, a single-null divertor tokamak run by GA Technologies in San Diego, California, a thermal quench of $\leq 0.2 \mathrm{~ms}$ has been observed [2.27]. Based on such recent experimental observation and the recommended values from the TIBER, INTOR, and ITER scoping studies, a benchmark value of 0.1 ms is assumed for this analysis. Based on these paraneters for the disruption, TADDPAK predicts heat fluxes ranging from $3.7 \times 10^{4}$ to $1.0 \times 10^{5} \mathrm{MW} / \mathrm{m}^{2}$.

\subsection{Plasma Transport in the Scrape-Off Layer}

During normal operations particles diffuse out of the magnetically confined plasina into the edge region called the scrape-off layer (SOI). Once this aif fusion has occurred, the parallel and perpendicular transport in the SOL play it significant role in the eventual distribution of power at the divertor surface. 


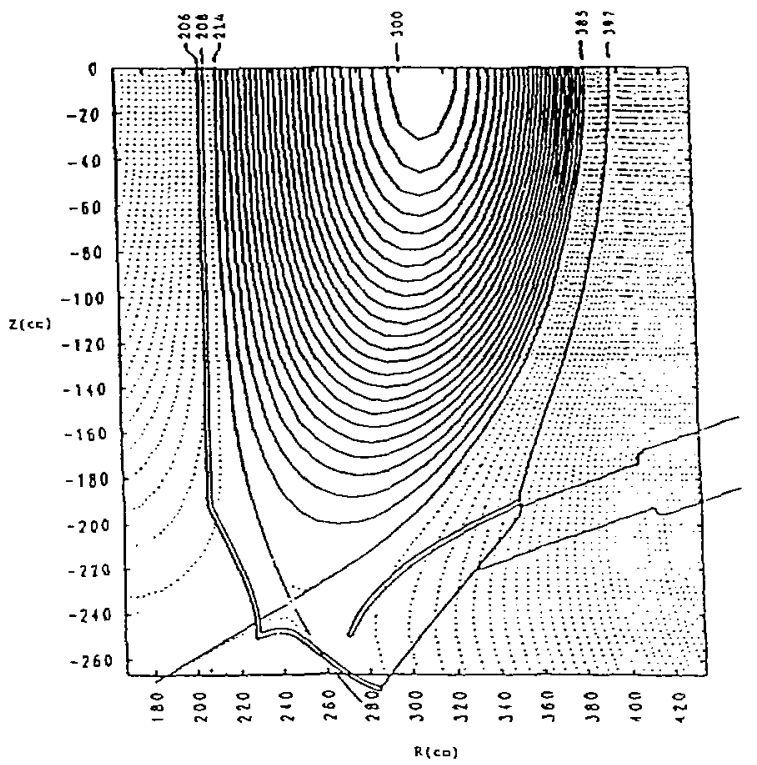

Figure 2.1: Magnetic topography of a poloidal cross section of the TIBER Il design.

Even more important in this process is the magnetic topography present in this region. Figure 2.1 illustrates the magnetic surfaces in a poloidal cross section of the TIBER II ciesign. What should be noted, although il is not readily apparent in this figure, is that the poloidally depicted magnetic surfaces are projections of the actual surfaces in three dimensions. The true connection length of each field line extends geometrically around the torus and may make several revolutions before completing its path. The helicity that results from the combination of toroidal and poloidal magnetic fields is important when considering the com- 
plete transport process.

As shown in Figure 2.1, the separatrix defines the edge of the plasma. Inside this boundary the magnetic field lines are closed. Here, parallel transport does not cause a loss of power since closure maintains plasma confinement. However, outside this boundary the flux surfaces diverge and spread out non-uniformly as they pass through the different sections of the divertor. Particles escaping from the plasma through perpendicular transport can readily flow along the SOL's open magnetic flux tubes and eventually impart their energy to the divertor surface. Such energy deposition results in significant surface damage to the tacing component. Therefore, it is important to be able to model the particle flow or heat flux in the SOL.

One of the first steps in modeling this process is to assume a direct correlation between the distribution of power and the distribution of particle density outside the separatrix. This assumption is reasonable since the magnetic field forces charged particles to follow its divergent field lines in the SOL. Hence, the more ions following a particular field line, the more energy deposited where that field line intersects the divertor. From further assuming one-dimensional particle diffusion in this region, as does Wagner in his study for ASDEX [2.28], the density distribution at the midplane would be given by

$$
n=n_{0} e^{-x / \lambda}
$$

where $n$ is the particle density as a function of the radial direction $\boldsymbol{x}$ out from the separatrix, $n_{0}$ is the density at the scparatrix, and $\lambda$ is the characteristic 
radial decay length. Since the particle density and power are assumed to be proportional, the power also can be expected to demonstrate the same exponential decay movin: outward from the separatrix. This assumption will be summarized in Chapter 4, where the power mapping routine of TADDPAK is discussed. In addition, the validity of applying this model to a disruption scenario will be addressed.

The characteristic radial decay length, $\lambda$, is an important parameter in this investigation. Since its value is an exponential folding constant in Equation 2.8 , it essentially determines the distribution of particles and, hence, energy on the divertor's surface. As the result of several recent theoretical efforts, models to determine the magnitude of $\lambda$ for steady-state operations have emerged. Harbour [2.29] estimates an average value for the radial decay length based on the ratio of stored energy to the actual power flow in the SOL. Stangeby [2.30] assumes uniform heat flow across the separatrix and obtains a simple formula for the thickness in terms of the connection length. More recently, Barr [2.31] developed an improved model, which includes the variation of thickness with the magnetic field strength along a flux tube. This magnetic field variation is illustrated in Figure 2.]. Barr estimates the thickness by

$$
\lambda=\sqrt{\frac{\chi_{\perp} L_{p}}{g C_{\iota}}},
$$

where $\chi_{1}$ is the radial thermal diffusivity, $g$ is the energy deposited per incident ion, and $C$, is the sound speed in the plasma at the divertor. $L_{p}$ is the projected 
connection length and is given by

$$
L_{p}=\frac{B_{t}}{B_{p o}} \int_{n u l l}^{\text {null }}-\frac{R^{2} B_{p}}{R_{o}^{2} B_{p o}} \cdot d s_{p}
$$

where $B_{t}$ is the toroidal magnetic field, $B_{p o}$ is the poloidal magnetic field at the midplane, and $d s_{p}$ is the poloidal surface of integration. As Equation 2.10 illustrates, $L_{p}$ is not equal to the conventional connection length in the poloidal plane. This difference is due to the radial variation of $B_{p}$ along the flux line. However, the greatest uncertainty in $\lambda$ results from the uncertainty in $\chi_{\perp}$ whose value is estimated between 1 and $4 \mathrm{~m}^{2} / \mathrm{s}[2.29,2.30]$ for steady-state operations. Barr estimates $\lambda$ to be approximately $1.5 \mathrm{~cm}$ for TIBER in steady state. Using DSTAR, Merrill [2.32] predicts a disruption $e$-folding for power of at least $3.0 \mathrm{~cm}$. The exact magnitude and time dependence of $\lambda$ during a disruption is not known, and little theoretical investigation has been made in this area. However, it is apparent that the diffusion coefficient must be greatly enhanced during a disruption to allow the intense particle flow from the core of the plasma. Therefore, $\lambda$ must also increase proportionally in magnitude according to Equation 2.9. Although no effort will be made in this thesis to theoretically evaluate the functional dependence of the characteristic decay length, the effects of varying its magnitude are presented in Chapter 5.

The last point to be made here is that the present analysis is for a disruption scenario and not for steady state. This conflict is resolved by the assumption that the steady-state approximations for transport in the SOL hold true during the thermal quencl phase of the disruption. The basis for this assumption and 
the other key assumptions in this analysis are discussed in detail in Section 4.2.

\subsection{Description of Disruption Computational Models (TSC, DSTAR)}

To simulate the edge transport discussed in the previous section, the disruption power flow from the bulk plasma must be known. The rate at which energetic particles move into the SOL during the thermal quench of a disruption eventually determines the thermal effects at the divertor surface. DSTAR [2.33], a computational model developed by Merrill to quantify the surface erosion and induced forces during a major plasma disruption, can simulate this power flow as a function of time. This code represents a comprehensive effort to model all aspects of the disruption event to include:

- Axisymmetric evolution of the plasma and background magnetic fields.

- Developinent of plasma and structural currents.

- Transport of plasma particles and energy.

- Growth of the stochastic region of the disrupting plasma.

- Melting and vaporizing of structural surfaces.

- Nonequilibrium ionization of, and emitted radiation from, the vaporized structural material as it is transported throughout the tokamak. 
Merrill accomplished this modeling effort by coupling the Tokamak Simulation Code (TSC) [2.34] with other models he developed to predict impurity transport and radiation and the electromagnetic and thermal dynamic response of vacuum vessel components. TSC, developed at the Princeton Plasma Physics Laboratory, predicts the evolution of the magnetic field and the transport of the plasma during the disruption. The TSC solves the coupled MHD and Maxwell's equations for the evolution of a tokamak plasma on a time scale that is representative of resistive plasma transport.

Despite this detailed treatment of simulating a disruption, DSTAR could provide a more efficient analysis in the divertor region. The lack of a computational mesh in this region leaves room for improvement. As noted in the introduction, this somewhat limited aspect of the code's detailed analysis is one reason for the development of TADDPAK. However, the main reason for developing TADDPAK is its ability to provide quick output for modeling many different facets of the divertor-plasma interaction. For exarnple, a parametric study looking at four different materials, pulse shapes, and characteristic decay lengths would take DSTAR approximately

$$
4 \times 4 \times 4 \times 45 \text { minutes } / \text { run }=48 \text { hours }
$$

while TADDPAK would only take

$$
4 \times 4 \times 4 \times 6 \text { minutes } / \text { run }=6.4 \text { hours }
$$


This time savings is even more pronounced when considering different machine geornetries.

Despite of this criticism, one mode of running TADDPAK still depends on DSTAR for its main input. DSTAR generated the power flow distribution illustrated in Figure 2.2 for a TIBER II disruption scenario of $0.1 \mathrm{~ms}$ and approximately $105 \mathrm{MJ}$ of stored thermal energy. This simulated, initially spiked, and exponentially decaying pulse shape is supported by recently observed disruptions in current experimental tokamaks [2.35]. The notch in the otherwise smooth decaying curve is just an anonalous effect of the code's calculations. The significance of using this form of heat flux pulse shape in this analysis is addressed in Chapters 4 and 5. 


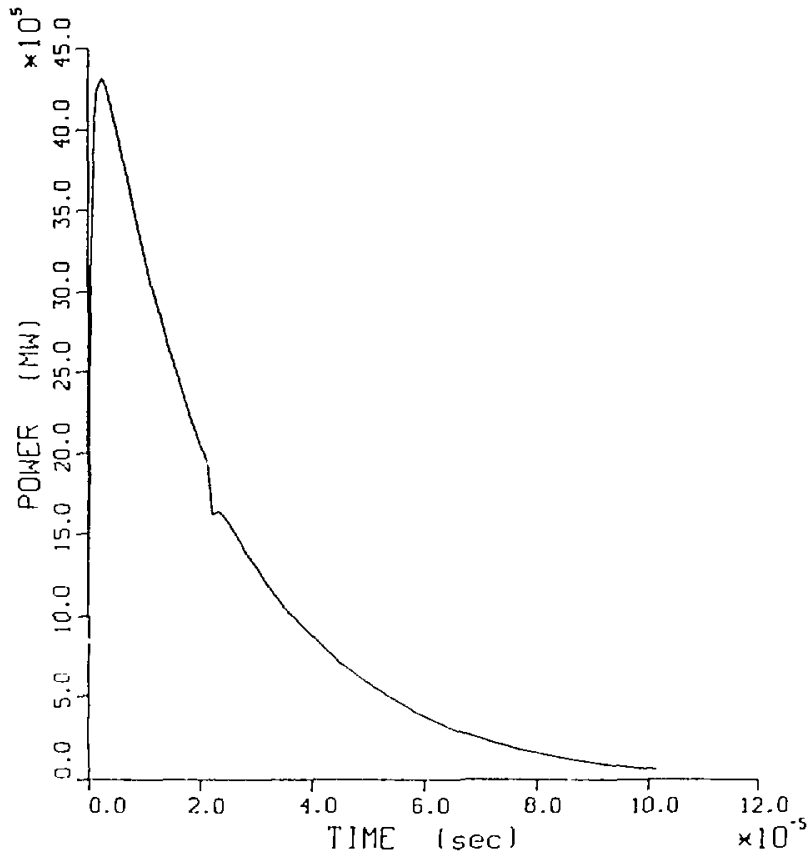

Figure 2.2: Disruption power flow as a function of time generated by DSTAR for a 0.1-ins thermal quench of 105 MJ. 


\subsection{References for Chapter 2}

2.1 G. Baternan, MHD Instabilities (MIT Press, Cambridge, MA, 1978).

2.2 J.A. Wesson, "Hydromagnetic Stability of Tokamaks," Nuclear Fusion, 18 (1978) 87-132.

2.3 S.V. Mirnov and I.B. Semenov, "Investigation of the instabilities of the Plasma String in the Tokamak-3 System by Means of a Correlation Method," Soviet Journal of Atomic Energy, 30 (1971) 22-29.

2.4 S. von Goeler et al., Physical Review Letters, 33 (1974) 1201-1203.

2.5 E.P. Gorbunov and K.A. Razumova, "The Effect of a Strong Magnetic Field on the Magnetohydrodynamic Stability of Plasma and the Containment of Charged Particles in the Tokamak," Soviet Journal of Atomic Energy, 15 (1963) 1105-1112.

2.6 R.B White et al., "Simulations of Large Magnetic Islands: A Possible Mechanism for a Major Tokamak Disruption," Physical Review Letters, 39 (1977) 1618.

2.7 J.W. Connor and J.B. Taylor, "Scaling Laws for Plasma Confinement," Nuclear Fusion, 17 (1977) 1047.

2.8 B.V. Waddel et al., "Nonlinear Interaction of Tearing Modes in Highly" Resistive Tokamaks," Physics of Fluids 22 (1979) 896.

2.9 B. Carreras et al., "Poloidal Magnetic Field Fluctuations," Nuclear Fusion, 19 (1979) 1423.

2.10 A. Sykes and J.A. Wesson, "Major Disruptions in Tokamaks," Physical Review Letters, 44 (1980) 1215.

2.11 K. Toi et al., "Soft and Hard Major Disruptions in Profile Control Experiment of the JIPP T-1I Tokamak," Nuclear Fusion, 22 (1982) 465.

2.12 M.F. Turner and J.A. Wesson, "Transport, Instability and Disruptions in Tokamaks," Nuclear Fusion, 22 (1982) 1069.

2.13 S. Tsuji et al., "Partial and Major Disruptions in the JIPP T-11," Nuclear Fusion, 25 (1985) 305-319.

2.14 F. Karger et al., in Plasma Physics and Controlled Nuclear Fusion Research 1976 (Proc. 6th Int. Conf. Berchtesgaden, 1976), Vol. 1, IAEA, Vienna (1977) 267. 
2.15 Equipe TFR, "Structure of low-Frequency Oscillations during the Disruptive Instability in the TFR Tokamak," Nuclear Fusion, 17 (1977) 1283.

2.16 TFR Group, "Low- $q$ MHD Activity Studies on TFR," Nuclear Fusion, 24 (1984) 784 .

2.17 S.V. Mirnov and I.B. Semenov, in Plasma Physics and Controlled Nuclear Fusion Research 1976 (Proc. 6th Int. Conf. Berchtesgaden, 1976), Vol. 1, IAEA, Vienna (1977) 291.

2.18 N.F. Sauthoff, "A Study of Disruptive Instabilities in the PLT Tokamak using X-Ray Techniques," Nuclear Fusion, 18 (1978) 1445.

2.19 B.V. Waddell et al., "Mechanism for Major Disruptions in Tokamaks," Physical Review Letters, 41 (1978) 1386.

2.20 K. Toi et al., "Current Density Profile Control by Programming of Gas Puffing and Plasma Current Waveform in JIPP T-11 Tokamak," Nuclear Fusion, 19 (1979) 1643.

2.21 K.M. McGuire and D.C. Robinson, "Major Disruptions in the TOSCA Tokamak," Physical Review Letters, 44 (1980) 1666.

2.22 "USSR Contribution to the INTOR Phase Two A Part 2 Workshop," Rep. Kurchatov Institute, Moscow (1985).

2.23 B. Merrill, Idaho National Enginecring Laboratory, EG\&G, private comInunication (August 1987).

2.24 R. Albarese, M. Biggio et a]., "NET Status Report," Commission of the European Communities Directorate General XII-Fusion Programme, Brussels, West Germany (1985) 333.

2.25 Report of the International Tokamak Reactor (INTOR) Workshop Phase Two A Part II, International Atomic Energy Agency (IAEA), Vienna, 1986.

2.26 R. Parker et al., CIT Physics Design Description, Princeton Plasma Phy'sics Laboratory (AE-880112 PPL-01) (January' 1988).

2.27 A.G. Kellman, "GA Technologies Inc. Contributions to the Specialists Meeting on Plasma Disruptions," IAEA, Vienna, 27-28 July 1987.

2.28 F. Wagner, "A Study of the Perpendicular Particle Transport Properties in the Scrape-off Layer of ASDEX," Nuclear Fusion, 25 (1985) 525-536. 
2.29 P.J. Harbour, "Thickness of the Scrape-off Layer of a Large Tokamak," Nuclear Fusion 24, 1211 (1984).

2.30 P.C. Stangeby, "The Plasma Sheath," in Physics of Plasma-Wall interactions in Controlled Fusion, Edited by D.E. Post and R. Behrisch, (Plenum Publishing Corp., New York 1986).

2.31 W. Barr, "The Thickness for Power Flow in TIBER's Edge Plasma," Lawrence Livermore National Laboratory internal memo (February 23, 1987).

2.32 B.J. Merrill, Idaho National Engineering Laboratory, EG\&G, private commurication (1987).

2.33 B.J. Merrill and S.C. Jardin, “User's Manual for DSTAR: A Comprehensive Tokamak Disruption Code," Idaho National Engineering Laboratory, EGG-EP-7394 (October 14, 1986).

2.34 S.C. Jardin et al., "Dynamic Modeling of Transport and Positional Control of Tokamaks," Princeton Plasma Physics Laboratory, Report PPPL-2258 (1985).

2.35 M. Ulriekson, "Contributions to the Specialists Meeting on Plasma Disruptions," IAEA, Vienna, 27-28 July 1987. 


\section{Chapter 3}

\section{Energy Deposition Theory}

\subsection{Thermal Response of Divertor Surface}

Once the distribution of disruption power at the divertor surface is estimated, the plasma-divertor interaction must be considered. This interaction determines the overall thermal response of the divertor material. Understanding the fun. damental processes involved during this intense energy deposition is necessary to derive a reasonable analytical model of this event. According to Hassanein [3.1], the plasma disruption-material interaction is divided into eight specific phases. Figure 3.1 summarizes the process using Hassanein's terminology with the following phase descriptions:

Phase 1 Prior to the plasma disruption, the divertor is characterized by a stcady-state temperature distribution $T_{S S}$, which results from the steadystate heat flux $F_{S S}$ and the internal cooling systern of the divertor. 


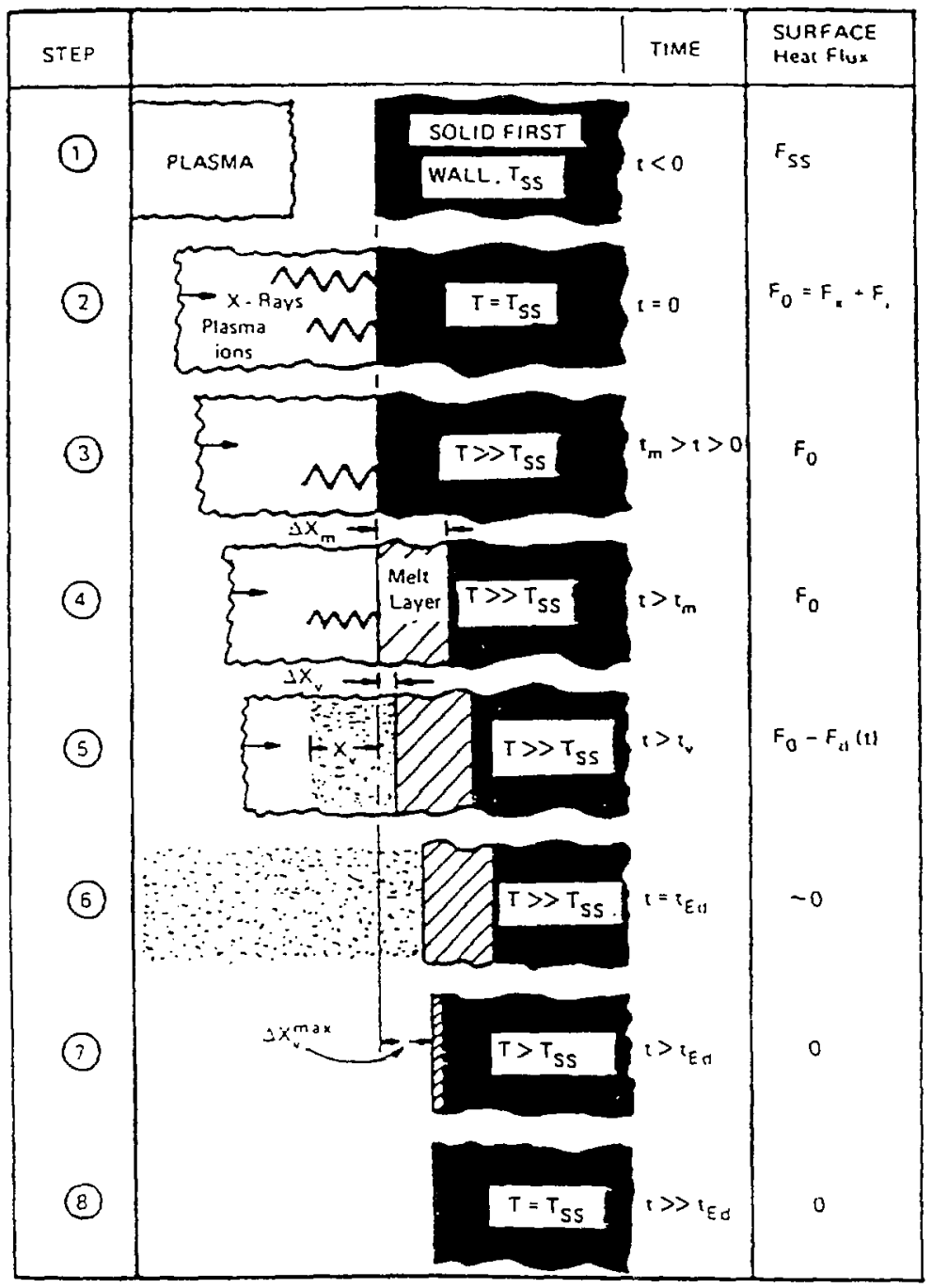

Figure 3.1: Schematic representation of plasma disruption-material interaction (fram reference i3.1!). 
Phase 2 At time $t=0$, the disruption begins and the heat flux increases abruptly to $F_{0}=F_{i}-F_{x}$, where $F_{1}$ and $F_{x}$ are the heat flux contributions resulting from parlicles and X-rays, respectively.

Phase 3 The surface temperature $T$ rises rapidly through conduction until sufficient latent heat has been absorbed to reach the melting point at time $t_{m}$.

Phase 4 The melt layer thickness $x_{m}$ increases as the liquid-solid interface penetrates deeper into the material. This boundary movement continues as more latent heat is absorbed. At the same time, the temperature of the liquid and the solid phases continues to rise as a result of heat conduction.

Phase 5 The vapor pressure of the liquid intensifies and significant evaporation can occur. The thickness of the material raporized $x_{v}$ depends on the amount of time the energy deposition continues after the beginning of vaporization at time $t_{\mathrm{u}}$. Also during this phase, the heat flux is reduced by an amount $F_{d}(t)$, which results frum the vaporized material absorbing the incoming energy and reradiating it isotropically. The surface temperature reaches a maximum where the rates of vaporization. melting, conduction, and radiation balance the total energy of the system.

Phase 6 The disruption has ended with a total duration of $t_{E d}$. The hot melt layer corls by vaporization. radiation, and conduction. Significant erosion can still uccur during this phase. 
Phase 7 The melt layer has cooled sufficiently to terminate significant vaporization. Resolidification of the liquid surface continues by heat conduction into the solid.

Phase 8 Resolidification is complete. The solid continues to cool by heat conduction until a steady-state temperature is reached.

For this scenario, the amount of vaporized material, the thickness of the melt layer, and the temperature distribution through the divertor can be determined through the proper formulation of the energy deposition process. This formulation can be accomplished by coupling the correct heat conduction equations for the different phases, selecting the proper boundary conditions, and utilizing appropriate numerical methods.

\subsection{The Heat Conduction Problem with Mov- ing Boundaries}

Since 1975 , several models $[3.2-3.6]$ have been developed to quantify the process illustrated in Figure 3.1. However, Hassanein was the first to formulate the complete heat conduction equation with two moving boundaries while still accounting for the temperature dependence of material properties and the $\mathrm{dy}$ namics of vaporization. Recent experiments of intense energy deposition [3.7] indicate that his model predicts the actual thermal response fairly accurately.

Hassanein treats the surface of the material as a semi-infinite solid and solves the problem in one dimension. This procedure is reasonable since the 
actual heat penetration depth is smal] compared to the linear dimensions of the surface. He also assumes the melt layer is stable and is not affected by magnetic or gravitational forces. This assumption may not be true since a liquid surface may conduct current and hence may experience a strong $\vec{J} \times \vec{B}$ force. The resulting instability could significantly affect the overall heat transfer in the surface. For the analysis presented in this thesis, however, we follow Hassanein's assumption. His formulation of the hat conduction equations, quantifying the processes depicted in Figure 3.1, is presented here in detail.

\subsubsection{Before Melting}

Under a disruption heat flux $F(t)$, the temperature profile $T_{0}(x, t)$ in the solid surface of the divertor must satisfy the heat conduction equation,

$$
\rho_{2} C_{0} \frac{\partial T_{2}(x, t)}{\partial t}-\nabla \cdot k_{,} \nabla T_{2}(x, t)=0
$$

where:

$$
\begin{aligned}
& \rho_{\varepsilon}=\text { solid density }\left(\mathrm{kg} / \mathrm{m}^{3}\right) ; \\
& C_{0}=\text { solid specific heat }(\mathrm{J} / \mathrm{kg}-\mathrm{K}) ; \\
& k_{\mathrm{a}}=\text { solid thermal conductivity }(\mathrm{J} / \mathrm{m}-\mathrm{s}-\mathrm{K} \mathrm{j}) ;
\end{aligned}
$$

Note that Equation3.1 has no volumetric energy deposition term since the heat flux in this problem is treated as purely a surface effect. All thermophysical properties for the materials of choice are presented in Section 4.3. Since these properties are functions of the local temperature, Equation 3.1 is nonlinear. This equation is valid during Phases $1,2,3$, and 8 as defined in Figure 3.]. 
When the energy deposition begins, Equation 3.1 is subjected to the following boundary conditions:

$$
\begin{aligned}
& T_{.}(x, 0)=T_{o}, \\
& T_{0}(x, 1) \longrightarrow T_{0} \text { as } x \rightarrow \infty,
\end{aligned}
$$

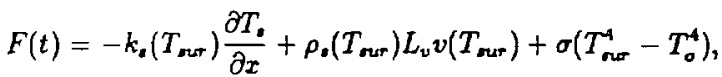

where:

$$
\begin{aligned}
& F(t)=\text { surface heat flux }\left(\mathrm{J} / \mathrm{m}^{2}-\mathrm{sec}\right) ; \\
& T_{\text {our }}=T_{\bullet}(0, t) \text { the surface temperature }(\mathrm{K}) ; \\
& L_{v}=\text { heat of vaporization }(\mathrm{J} / \mathrm{kg}) ; \\
& v\left(T_{\text {sur }}\right)=\text { velocity of receding surface }(\mathrm{m} / \mathrm{s}) ; \\
& \sigma=\text { Stefan-Boltzmann constant }\left(\mathrm{J} / \mathrm{K}^{4}-\mathrm{m}^{2}-\mathrm{s}\right) ; \\
& T_{\mathrm{o}}=\text { steady-state temperature }(\mathrm{K}) .
\end{aligned}
$$

Equation 3.4 represents the energy balance at the surface of the divertor. The incident heat flux $F(t)$ is divided among three surface phenomenon. The first term accounts for the heat removed from the surface by conduction through the solid. The second term represents the energy consumed during the vaporization process as the temperature increases. This term is insignificant at temperatures below the melting temperature of the material. However, it becomes dominant in this equation at elevated temperatures. The last term accounts for the radiative heat transfer from the surface back into the vacuum vessel. Usually the vaporization term limits the maximum temperature enough so that beat loss from radiation never becomes significant. 


\subsubsection{During and After Melting}

After melting begins, all three phases (solid, liquid, and rapor) of the material are present at the surface. Figure 3.2 illustrates the three regions and their interfaces. The boundaries are defined as:

$$
\begin{aligned}
& s(t)=\text { location of the vapor/liquid interface, } \\
& m(t)=\text { location of the solid/liquid interface. }
\end{aligned}
$$

The heat equation in the melt layer is given by:

$$
\rho_{l} C_{l} \frac{\partial T_{l}(x, t)}{\partial t}-\nabla \cdot k_{l} \nabla T_{l}(x, t)=\mathbf{0}
$$

where each variable has the subscript $l$, indicating the liquid phase. Since the temperature is continuous at the liquid/solid interface, this equation is subject to the following boundary conditions:

$$
T_{\iota}(x, t)=T_{l}(x, t)=T_{m}
$$

which is valid at $x=m(t) . T_{m}$ is the melting or solidification temperature of the material. Energy also must be conserved at this boundary. The appropriate energy constraint is given by:

$$
-\left.k_{i} \frac{\partial T_{i}}{\partial x}\right|_{m(t)}=-\left.k_{i} \frac{\partial T_{i}}{\partial x}\right|_{m(t)}+p_{0} L_{f} \frac{d m(t)}{d t}
$$

where $L_{f}$ is the latent heat of fusion. Equation 3.7 stipulates that the heat flow :n the liquid must equal the heat flow in the solid less the heat of fusion consumed by the advancing solid/liguid interface. Noted that in Equation 3.7, the quantity $\frac{d m(t)}{d t}$ represents the velocity of this interface. 


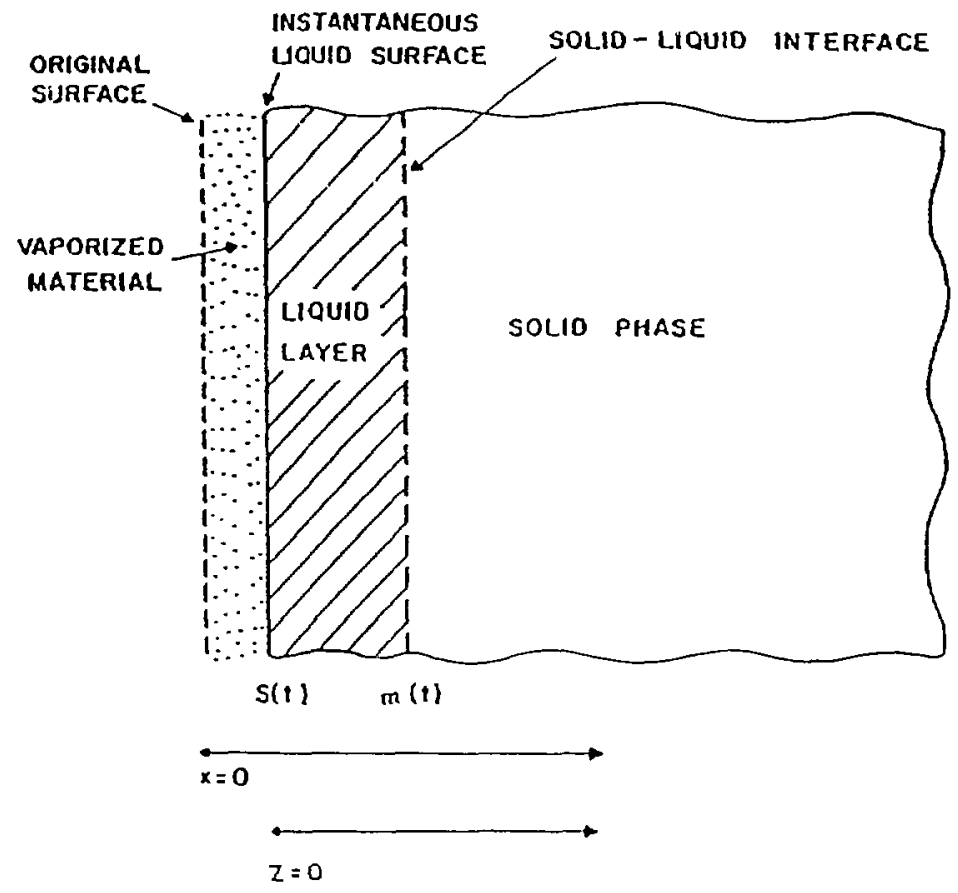

Figure 3.2: Schematic representation of the solid, liquid, and rapor phases (from Reference [3.1]). 
The boundary condition at the vapor/liquid interface, $x=s(t)$, is now:

$$
F(t)=-k_{l}\left(T_{\text {sur }}\right) \frac{\partial T_{l}}{\partial x}+\rho_{l}\left(T_{\text {our }}\right) L_{v} v\left(T_{\text {our }}\right)+\sigma\left(T_{\text {rerr }}^{4}-T_{o}^{-4}\right)
$$

\subsubsection{Moving Boundary caused by Evaporation}

The intense energy deposition occurring during the course of a disruption will almost immediately cause significant vaporization at the surface of the divertor. This surface movement must be accounted for within the set of heat conduction equations. Considering this movement is especially important since the surface may traverse nodal points of the finite differencing mesh used in calculating a numerical solution. Such a requirement can be accomplished by introducing a moving coordinate system:

$$
Z(t)=x-s(t)
$$

such that the surface always remains at $Z=0$. Making this coordinate transformation and using

$$
\frac{\partial Z}{\partial t}=-\frac{\partial s(t)}{\partial t}=-v(t)
$$

Equations 3.1 and 3.5 yield

$$
\rho C \frac{\partial T}{\partial t}-\rho C v(t) \frac{\partial T}{\partial Z}-\nabla \cdot k \nabla T=\dot{q}(z, t)
$$

Hassenein is among many to solve the heat conduction equation with moving boundaries [3.1]. His complete solution incorporates an evaporation model for determining both the surface heat flux, $F(t)$, and the receding surface velocity, $v(t)$. Note that the evaporated thickness can be determined by 
integrating the surface velocity, $v(t)$, over time. This evaporation model is discussed in Section 3.2.4. The phase of the region to which Equation 3.11 is applied, determines the appropriate thermophysical properties. The specific form of these properties is presented in Section 4.3.

\subsubsection{Evaporation Model}

Functions of the surface velocity and heat flux are required to solve to Equations 3.1 through 3.11. The Hertz-Kudsen-Langmuir theory of evaporation and condensation [3.8] provides the necessary expressions. According to thcir theor $y$, the evaporation flux of atoms from a condensed phase into a vacuum is given by:

$$
\begin{gathered}
J_{e}^{\text {vac }}=\left(2 \pi m k T_{\text {our }}\right)^{-\frac{1}{2}} \sigma P_{a}\left(T_{\text {rur }}\right), \\
P_{\bullet}=F_{\text {o }} \exp \left(-\Delta H / k T_{\text {eur }}\right)
\end{gathered}
$$

where: $m=$ atom mass;

$k=$ Boltzrnann constant;

$T_{\text {eur }}=$ surface temperature of the condensed phase;

$\sigma=$ coefficient to compensate for nonideal behavior;

$P_{0}=$ saturation vapor pressure;

$P_{o}=$ material dependent constant;

$\triangle H=$ enthalpy of vaporization. Equations 3.12 and 3.13 would be valid if the surface vapor density remained sufficiently low, as a result of vapor expansion into the racuum. However, in this problem, the vapor den- 
sity increases to the point of causing backscattering and recondensation at the surface. The actual evaporation flux, $J(t)$, is less than the value given by Equation 3.12. This specific transport problem was solved by Anisimov and Rakhmatulina. Their calculations [3.9] showed that the recondensation flux asymptotically approaches $80 \%$ of the vacuum evaporation flux, $J_{e}^{\text {vac }}$. Hence, the net evaporation fiux, $J(t)$, can be approximated by.

$$
J(t)=J_{e}^{v a c}\left(T_{\text {nur }}\right)\left[0.8+0.2 \exp \left(-t / \tau_{r}\right)\right]
$$

where $\tau_{r}$ is the relaxation time. This is the time required to reach the vapor density needed to cause full condensation at the surface after the start of evaporation. Using mean free path arguments, Hassanein et al. $[3.1-3.10]$ show that this time can be related to the vacuum evaporation flux by:

$$
\frac{1}{\tau_{r}}=2.73 \Omega^{\frac{2}{3}} J_{e}^{\text {vac }}
$$

where $\Omega=\frac{4}{3} \pi a_{0}^{3}$, the atomic volume of the diverlor material with an atomic radius $a_{o}$, is used as an approximation for the elastic scattering cross section. Using Equations 3.12 through 3.15, the velocity of the receding surface, $v(t)$, can now be determined as a function of temperature with the following equation:

$$
v(t)=\Omega J(t)
$$

With Equation 3.16, the complete heat conduction problem described in Section 3.2 .3 can be solved with the appropriate numerical methods. 


\subsection{Numerical Methods}

Because Equations 3.1 through 3.16 are nonlinear and the temperature is dependent on the material's thermophysical properties, there are no analytical solutions to this complex heat conduction problem. However, several finite differencing schemes are available for determining a numerical solution. Hassenein uses the modified implicit method of Crank and Nicolson [3.11] for his analysis. He chose this method because it gives stable solutions and involves less truncation error because a large number of space and time steps are required. The resulting system of simultaneous equations was solved by an implicit alternating direction method suggested by Peaceman and Rachford [3.12]. The nonlinear parameters, such as material properties and surface velocities, are reevaluated each time step to approximate a quasilinear system of equations that can be solved by these methods.

Hassanein wrote the computer code A*THERMAL [3.10], which solves this energy deposition problem in detail. His code models the rapidly varying volumetric energy depositions and accounts for multiple particle species heat flux and the transport of ablated material back into the plasma. This extensive multiparticle species model has been modified by Croessmann for single bombarding particle species in the code SOAST [3.7]. Croessmann's version of Hassanein's computer code is one of the important modules of TADDPAK. The single species version of this code suffices for this analysis for two reasons. First, the problem can be approximated by an "average particle" heat flux. Second, 
the distance of the divertor surface from the plasma edge precludes the need to account for the transport of ablated material back to the plasma edge other than treating vapor shielding at the material surface. Lse of this streamlined version also significantly decreases the required computation time. 


\subsection{References for Chapter 3}

3.1 A.M. Hassanein, G.L. Kulcinski, and W.G. Wolfer, "Surface Melting and Evaporation During Disruptions in Magnetic Fusion Reactors," Nuclear Engineering and Design/Fusion 1 (1984) 307.

3.2 J.G. Andrews and D.R. Atthey, J. Inst. Maths. Applics. 15 (1975) 59.

3.3 L.L. Loebel and W.G. Wolfer, "Evaporation Under Intense Energy Deposition," University of Wisconsin Fusion Engineering Program Report LWFDM-370 (August 1980).

3.4 R.J. Onega and B.M. Su, "Neutronics, Photonic, and Thermal Calculations Relevant to a Major Plasma Disruption in a Canister-Type Tokamak Blanket," Nuclear Technology/Fusion 2 (1982) 667.

3.5 J.A. Fillo and H. Makowitz, "Heat Transfer Modeling of First Walls Subject to Plasma Disruption," Engineering Problems of Fusion Research 2 (1981) 1775 .

3.6 A.D. Bowers and J.R. Hajnes, "Response of Fusion Reactor First Walls to Major Plasma Disruption," Journal of Nuclear Materials 103/104 (1981) 81 .

3.7 C.D. Croessmann, "The Thermal Response of Structural Materials to Intense Energy Deposition," Ph.D. thesis, University of Wisconsin-Madison (1986) (UWFDM-674).

3.8 J.P. Hirth and G.M. Pound, Progress in Material Sciences II Pergamon Press, New York. (1963).

3.9 S.I. Anisimov and A.K. Rakhmatulina, "The Dynamics of the Expansion of a Vapor when Evaporated into a Vacuum," Soviet Physics JETP 37(3) (1973) 441.

3.10 A.M. Hassanein, G.L. Kulcinski, and W.G. Wolfer, "Vaporization and Melting of Materials in Fusion Devices," Journal of Nuclear Materials $103 / 104$ (1981) 321 .

3.11 J. Crank and P. Nicolson, "A Practical Method for Numerical Evaluation of Solutions of Partia] Differential Equations of the Heat-Conduction Type," Proceedings of the Cambridge Philosophical Society 43 (1947) 50.

3.12 D. II. Peaceman and H.H. Rachford, "The Numerical Solution of Parabolic and Elliptic Differential Equations," Joumal of the Society for Industrial and Applied Mathematics 3(1) (1955) 28. 


\section{Chapter 4}

\section{TADDPAK}

\subsection{Description}

TADDPAK is a semiportable FORTRAN computer code package designed for modeling the thermal response of the divertor in a doublc-null configured tokamak during the thermal quench of a major plasma disruption. It is semiportable in that it will run on any mainframe system which utjlizes FORTRAN $V$ and which has the graphic libraries and plotting routines necessary for the code's output. Specifically, these software requirements are GRAFLIB [4.1] and DISSPLA 14.2]. For this thesis TADDPAK was run on a CRAY-2 supercomputer belonging to the Magnetic Fusion Energy Division of Lawrence Livermore National Laboratory: The CRAY-2 was used only to take advantage of the graphics feat ures available from DISSPLA not - because of computational requirements.

The basic structure of TADDPAK is illustrated in Figure 4.1. Two forms of input are available for use with this code package. DSTAR can provide the profile of the power leaving the edge of the plasma during a disruption, or a 


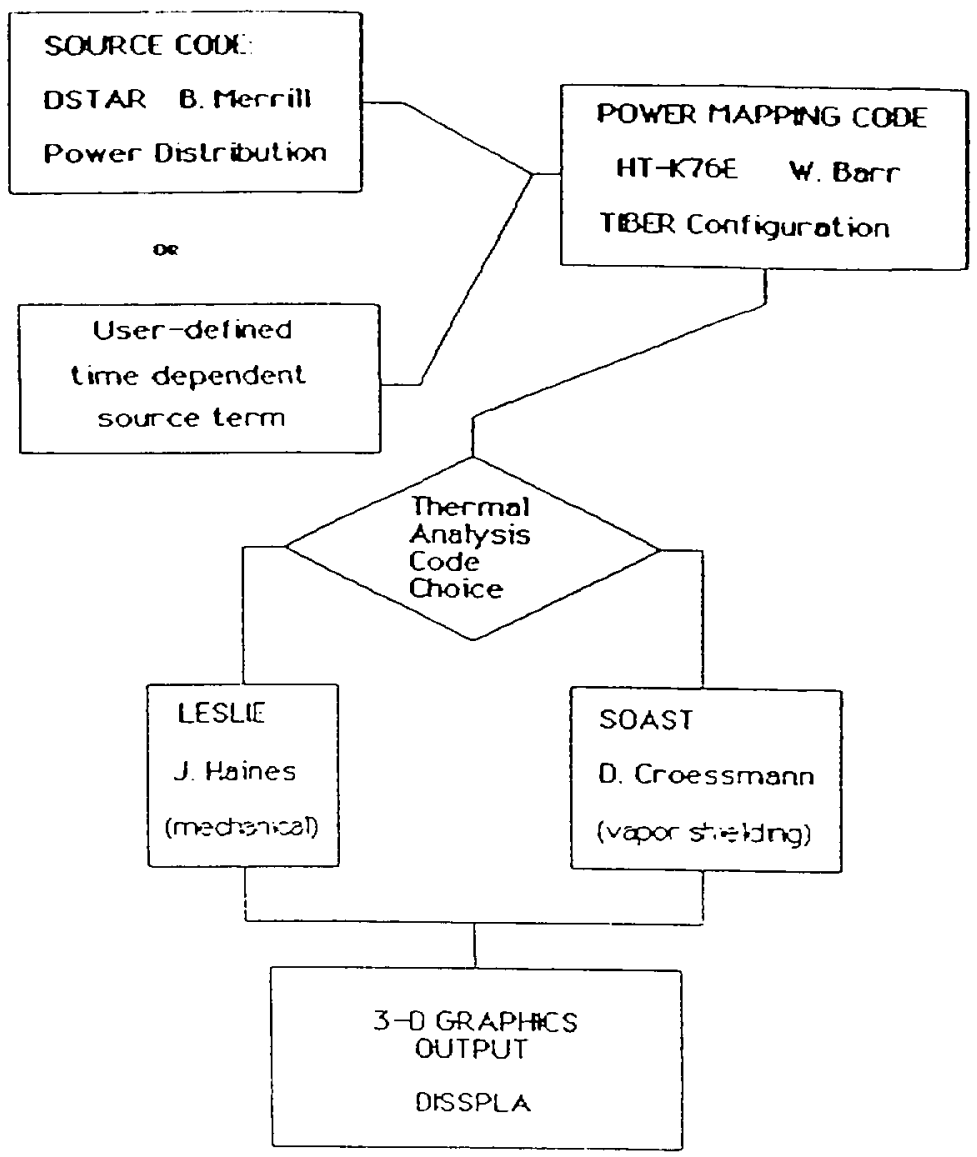

Figure 4.1: TADDPAK flow chart structure. 
user-defined profile can be used. The user-defined profile could be based on either current experimental data or user preference. This feature of the code, as mentioned in Section 2.5, is a significant improvement over past analyses [4.3-4.8, which evaluated only square and triangular disruption pulse shapes. Using a profile that more closely approximates the actual time dependence of a disruption can result in significant differences in the overall thermal dynamic response of the divertor. The similarities and differences will be presented in the Chapter 5. For the analysis presented in this thesis, a DSTAR-generated profile, previously illustrated in Figure 2.2, is used for all calculations except those comparing a square pulse shape. The DSTAR profile was used for input because of its close approximation to recent experimental observations, the availability of its specific numerical form, and the total stored thermal energy it represents for the TIBER Il reactor design. Relying on DSTAR for this profile does not increase the running time of TADDPAK since the profile has already been extracted from the code's previously documented results for TIBER II.

Using either the DSTAR profile or a user-defined profile as input, the first module of TADDPAK calculates the time- and position-dependent power densities on a poloidal cross section of the divertor. This calculation is accornplished by mapping the disruption energy flow from the edge of the plasma to the divertor surface. The original version of this module was the code HT-K76E 14.9 , a power-mapping computer program originally written by $W$. Barr to assist in divertor design under steady-state conditions. This program embodies 
the physics of particle/energy transport in the SOL as discussed in Section 2.4. Barr's IBM basic version has been translated into FORTRAN, adapted for the time-dependent problem of a plasma disruption, and coupled to a thermal analysis module.

Once the time-dependent power density distributions due to the particle flux are determined, the corresponding distributions caused by isotropic radiation must be added to this distribution for a complete calculation. With this total distribution, the resulting thermal response on each divertor plate can be calculated by one of two thermal analysis modules. These modules are LESLIE [4.10], written by J. Haines, and SOAST [4.11], written by D. Croessmann. Both codes solve the moving boundary heat equation in one dimension for each partitioned segment of the divertor. In addition to providing one-dimensional thermal analysis, LESLIE can calculate mechanical effects to include stress and strain. Since this thesis focuses only on the thermal effects, SOAST, an abridged version of the comprehensive A*THERMAL code, is the module used. SOAST provides thrsmal analysis comparable to LESLIE and offers a simple vapor-shielding model. The output of the selected code is then integrated into DISSPLA which generates high-quality three-dimensional graphics of chosen position- and time-dependent varjables for the inner, lower, and outer divertor plates. The polojdal configuration for the inner, lower, and outer divertor plates was illustrated in Figure 2.1. The selected variables include surace temperature, vaporization rate, thickness of material ablated, and total 
volume and mass ablated from the entire divertor surface. From this output, the relative peaking factors on the divertor plates can be determined.

The other feature of TADDPAK that merits mention is its generic design. Through minor modifications, this code package can easily be adapted for other single- or double-null divertor reactor designs. In fact, it has already been used to analyze the ITER reactor design. A limited selection of these resuss are presented in the appendix. The ma:n in put data required by TADDPAK are the plasma equilibrium magnetic flux surfaces for a poloidal cross section outside the SOL, the divertor coordinates, the material choice, and the calculated plasma's stored thermal energy. Also for the particular design configuration, assumptions must be made for the partitioning of disruption energy in the different regions of the SOL. Once these input data have been determined, TADDPAK can be implemented easily.

\subsection{Assumptions}

TADDPAK's simplicity and origin result from several key underlying assumptions. First, and probably most important, is the assumption that particle transport in the SOL can be approximated by steady-state physics during the thermal quench of a major disruption. This statement implies that the equilibrium poloidal magnetic flux surfaces used by the power-mapping routine do not significantly change position or shape during the first 100 microseconds of a disruption. Therefore, the power densities on the divertor can be assumed to 
have a distribution proportionately similar to the distribution present during steady state. However, the time dependence of the disruption power flow must be included in such a calculation.

This claim is based on the long L/R time, or in other words, on the strong inductive nature of the plasma and its adjacent and highly conducting containment vessel [4.34]. To evaluate this e-folding time, the diffusion of the magnetic field through the plasma and the first wall must be considered. The complete diffusion problem for the magnetic field of a confined plasma is complex since it involves the detailed analysis of an intricate circuit consisting of plasma, first wall, and numerous outer structural materials. A thorough calculation of the characteristic diffusion time goes beyond the scope of this thesis. However, the relative magnitude of this time can be inferred from the individual characteristic diffusion times of various components of the circuit. A valid approximation for this time, $\tau$, can be made using the following equation from reference [4.13]:

$$
\tau=\frac{\mu_{0} l^{2}}{\eta}
$$

where $\mu_{0}$ is the permeability of free space, $l$ is the skin depth, and $\eta$ is the material's resistivity. The skin depth for the TIBER II first wall is approximately $0.02 \mathrm{~m}$. Other structural materials outside the vacuum vessel have significantly larger characteristic thicknesses. The resistivity of a plasma was first given by Spitzer [4.14] as:

$$
\eta=1.65 \times 10^{-\theta} \frac{Z_{e j f} \ln \Lambda}{T_{e}^{3 / 2}}
$$


Table 4.1: Characteristic diffusion times of the magnetic field

\begin{tabular}{||l|c|c|}
\hline Material & $\begin{array}{c}\text { Electrical } \\
\text { Resistivity }(\mu \Omega-\mathrm{m})\end{array}$ & $\begin{array}{c}\text { Characteristic } \\
\text { Time }(\mathrm{ms})\end{array}$ \\
\hline Copper & 0.017 & 29.3 \\
Tungsten & 0.055 & 9.1 \\
Stainless Steel & 0.75 & 0.7 \\
Plasma & 0.004 & 8700 \\
\hline
\end{tabular}

where $Z_{e f f}$ is the effective charge of plasma impurities (typically taken as 1.5 ), $\ln \Lambda$ is the coulomb logarithm (approximately 18 ), and $T_{e}$ is the plasma electron temperatuse. TIBER 11 has an average electson temperature of $20 \mathrm{keV}$. During the thermal quench, this temperature will decrease rapidly. An average value of $5 \mathrm{keV}$ is assumed for use in Equation 4.2. For the plasma, $l$ in Equation 4.1 is the scale length of the spatial variation of the magnetic field. This value can be approximated by the inverse of the Taylor eigenvalue $1 / \lambda \sim r / 5$, where $r$ is the minor radius [4.15]. Evaluating Equations 4.1 and 4.2 for both candidatc firstwall materials and the plasma, yield the characteristic diffusion times shown in Table 4.1. The characteristic time of the entire system should fall somewhere within the range of these values. Except for stajnless steel, all of these values are at least an order of magnitude larger than the thermal quench time. Compared to the 0.1 -millisecond duration of the thermal quench, these longer characteristic times indicate that the first assumption is reasonably valid.

The assertion of quasistable magnetic flux surfaces in the SOL during the thermal quench of a disruption is further supported by calculations made by DSTAR. This code predicts that the separatrix and null point of the magnetic 
flux surfaces move only a few millimeters from their original positions during the first 0.1 inilliseconds of the disruption [4.16]. This minimal movement of the flux surfaces implies that the power density distributions present on the divertor surface during steady state will not change significantly during the thermal quench of a disruption. Therefore, since the partitioned spatial bins of the divertor surface are on the order of several centimeters, the resulting thermal response calculations should be representative of the actual processes occurring on the divertor surface. The partitioning of the divertor surface into spatial bins is the numerical technique used to enable the use of the one-dimensional thermal module previously discussed. This technique is based on the next assumption.

The second assumption is the belief that a one-dimensional model can be used to analyze the cross section of the divertor in two dimensions. This assumption is based on the fact that the partitioned divertor mesh spacings within the computer code are several orders of magnitude wider than the penetrating depth of the resulting thermal effects. This distance, known as the thermal diffusion length, can be written as

$$
l=\sqrt{\alpha t}
$$

where $\alpha$ is the thermal diffusivity and $t$ is the disruption time. For graphite, $\alpha=0.14 \mathrm{~cm}^{2} / \mathrm{s}$, which yields a diffusion length of $0.004 \mathrm{~cm}$ for $t=0.1 \mathrm{~ms}$. Compared to the $1-5 \mathrm{~cm}$ width of each divertor mesh segment, this small ther$\mathrm{mal}$ penetration allows the heat conduction along the surface of each spatial bin to be neglected. Hence, the application of this one-dimensional model is valid. 
Third, the partitioning of power in the SOL is assumed to be a 20:80 ratio between the inner and outer SOLs, respectively. As discussed in Section 2.4, this ratio has been adopted from the NET design study. Also, the power partitioning between upper and lower divertors is assumed to be equal, as a result of to the symmetry of the reactor design. All calculations made by TADDPAK are for one set of divertor plates, or half of the machine.

Fourth, the calculated particle flux incident on the divertor is assumed to deposit all of its energy. This assumption is conservative since the particles will experience some degree of backscattering depending on their energy. At the high energies present during the thermal quench of a disruption backscattering cross sections for hydrogen-like particles are not very large. In addition, since only heatfluxes are actually considered in these calculation, this conservative assumption is employed.

Last, throughout this analysis, the thermal quench of the disruption is assumed to occur axisymmetrically within the confines of the vacuum vessel. However, in reality no two disruptions result in exaztly the same distribution of power on the divertors. On the other hand, disruptions do demonstrate many similar characteristics. It is the hope of this analysis to provide a quick and reasonable estimate of this complex phenomena within the limitations of current understanding. 


\subsection{Material Properties}

This analysis focuses on three candidate materials for the divertor: graphite, tungsten, and beryllium. Throughout the development of the heat conduction problem in Chapter 3, the need to consider the temperature-dependence of the material's thermophysical properties was emphasized. Only tinrough such consideration car an accurate solution to the problem be obtained. The intense energy deposition of a disruption can cause temperature transients of more than several thousand degrees. Such temperature differences result in significant variations in certain thermophysical properties. To accommodate such radical temperature changes, the temperature-dependent properties are defined by an equation of the following functional form:

$$
f(T)=c_{0}+c_{1} T+c_{2} T^{2}+c_{3} \exp \left(-T / c_{4}\right)
$$

where $f(T)$ is either the specific heat or thermal conductivity, $c_{0}$ through $c_{4}$ are the appropriate coeffi.jents, and $T$ is the local temperature in degrees kelvin.

The ot tes important thermophysical property with significant temperature dependence is the saturation pressure. Equations 3.12 through 3.15 show that this pressure influences the energy balance significantly at the surface of the material. Expressing the proper pressure is achieved with an equation of the following functional form:

$$
P(T)=\exp \left(-a_{1} / T+a_{2}\right)
$$

where $a_{1}$ and $a_{2}$ are the coefficients of pressure variation and $P$ is pressure 
Table 4.2: Thermophysical properties and function constants

\begin{tabular}{|c|c|c|c|}
\hline \multirow[b]{2}{*}{ Property } & \multicolumn{3}{|c|}{ Material } \\
\hline & Graphite & Tungsten & Beryllium \\
\hline Density $\left(\mathrm{mg} / \mathrm{m}^{3}\right)$ & 1.74 & 19.3 & 1.85 \\
\hline \multicolumn{4}{|l|}{ Specific Heat $(\mathrm{J} / \mathrm{kg}-\mathrm{K})$} \\
\hline$c_{0}$ & 2100 & 128 & 1210 \\
\hline$c_{1}$ & - & 0.0249 & 1.34 \\
\hline$c_{2}$ & - & $1.43 \times 10^{-6}$ & - \\
\hline$c_{3}$ & -2292 & - & - \\
\hline$c_{4}$ & 600 & - & 一 \\
\hline \multicolumn{4}{|l|}{ Thermal Conductivity $(\mathrm{W} / \mathrm{m}-\mathrm{K})$} \\
\hline$c_{0}$ & 50 & 103 & 70 \\
\hline$c_{1}$ & - & - & - \\
\hline$c_{2}$ & - & - & - \\
\hline$c_{3}$ & 294 & 103 & 200 \\
\hline$c_{4}$ & 480 & 550 & 370 \\
\hline Melting Temperature (K) & - & 3683 & 1557 \\
\hline Latent Heat of Fusion $(\mathrm{MJ} / \mathrm{kg})$ & - & 0.184 & 1.083 \\
\hline Latent Heat of Vaporization $(\mathrm{MJ} / \mathrm{Kg}$ ) & 59.1 & 4.815 & 24.8 \\
\hline Liquid Density $\left(\mathrm{Mg} / \mathrm{m}^{3}\right)$ & - & 17.6 & 1.69 \\
\hline Liquid Specific Heat $(\mathrm{J} / \mathrm{kg}-\mathrm{K})$ & - & 193 & 3590 \\
\hline Liquid Thermal Conductivity (W/m-K) & - & 100 & 70 \\
\hline
\end{tabular}

in pascals. The numerical values of these coefficients and other thermophysical properties for graphite, tungsten, and beryllium are listed in Tables 4.2 and 4.3. Note that graphite does not have a liquid state within the pressures and temperatures of a disruption. These properties were obtained from references $[4.17-4.19]$. 
Table 4.3: Constants for saturation pressure function Equation 4.5

\begin{tabular}{||c|r|r|r|}
\hline & \multicolumn{3}{|c|}{ Material } \\
\cline { 2 - 4 } & Graphite & \multicolumn{1}{|c|}{ Tungsten } & Beryllium \\
\hline Pressure & & & \\
$a_{1}(\mathrm{~K})$ & $8.85 \times 10^{4}$ & $9.476 \times 10^{4}$ & $3.643 \times 10^{4}$ \\
$a_{2}$ & 32.1 & 27.4 & 24.75 \\
\hline
\end{tabular}

\subsection{Graphical Ot: put}

One of TADDPAK's valued features is its graphical versus numerical presentation of results. The three-dimensional graphics provide a quick way to analyze large amounts of numerical data. For each run, TADDPAK generates a separate set of plots for each divertor plate. An example is illustrated in Figure 4.2.

Since vertical symmetry is assumed, each plot represents the particular processes occurring in half of the machine. Any global values, such as volume and mass, must be multiplied by two. Time and distance are plotted on the $x-y$ plane of each plot. The chosen parameter is plotted on the $z$-axis. The distance along each plate is the linear distance from where energy deposition begins on the inboard side of the reactor. For the lower plate, this point is where the separatrix and the plate's surface intersect. When cumulative effects must be considered, they are projected onto the left side of the plot. 
INNER DIVERTOR PLATE
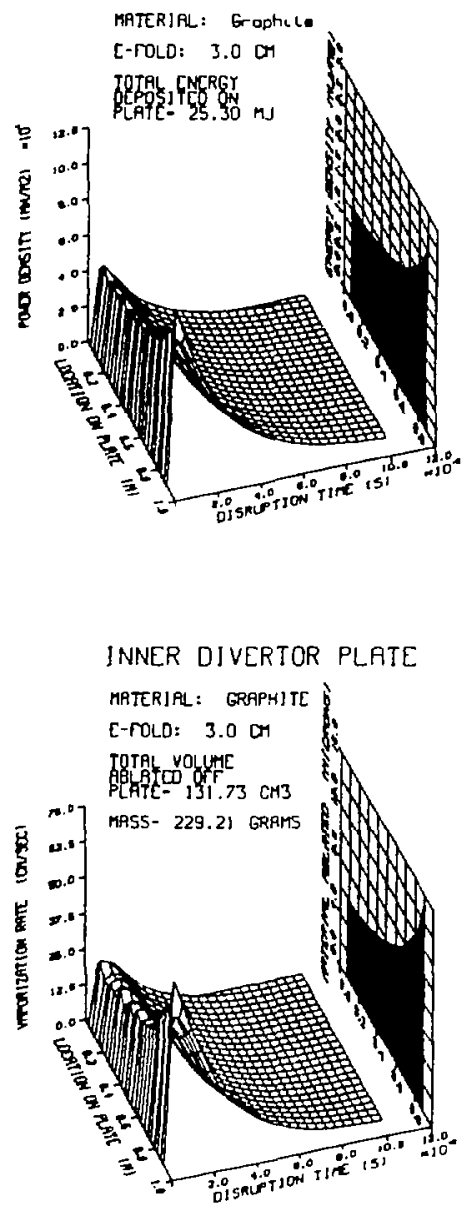

INNER DIVERTOR PLRTE

MATERIFL: GRAFHITE

[ ?LD: 3.0 CN

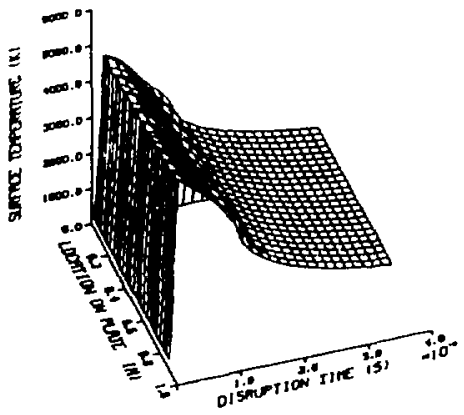

Figure 4.2: Example of TADDPAK graphical output. 


\subsection{References for Chapter 4}

4.1 K. O'Hair et al., GRAFLIB User's Manual, Livermore Computing Systems Document, Lawrence Livermore National Laboratory, LCSD-425 (January 1984).

4.2 Integrated Software Systems Corporation, DISSPLA (Display Integrated Software System and Plotting Language) User's Manual, (San Diego, California, 1981).

4.3 J.A. Fillo and H. Makowitz, "Heat Transfer Modeling of First Walls Subject to Plasma Disruption, ${ }^{n}$ Engineering Problems of Fusion Reseanch 2 (1981) 1775.

4.4 A.D. Bowers and J.R. Haines, "Response of Fusion Reactor First Walls to Major Plasma Disruption," Journal of Nuclear Materials 103/104 (1981) 81.

4.5 A.M. Hassanein, Thermal Effects and Erosion Rates Resulting from Intense Deposition of Energy in Fusion Reactor First Walls, Ph.D. thesis, University of Wisconsin-Madison (1982) (UWFDM-465).

4.6 A.M. Hassanein, G.L. Kulcinski, and W.G. Wolfer, "Surface Melting and Evaporation During Disruptions in Magnetic Fusion Reactors, ${ }^{n}$ Nuclear Engineering and Design/Fusion 1 (1984) 307.

4.7 J.A. Koski et al., "Preliminary Design Analysis of the ALT-II Limiter for TEXTOR," Journal of Nuclear Materials 121 (1984) 309.

4.8 M.F. Smith, "Low-Z Armor for the ALT-II Limiter," Sandia National Laboratories internal memo, Livermore, CA (July 12, 1983).

4.9 W.L. Barr, Lawrence Livermore National Laboratory, private communication (1987).

4.10 J.R. Haines, K. Kazunori, and T. Kobayashi, "Parametric Study of FER First Wall and Divertor Plate Performance," Japan Atomic Energy Research Institute, JAERI-M 86-099 (1986).

4.11 C.D. Croessmann, "The Thermal Response of Structural Materials to Intense Energy Deposition," Ph.D. thesis, University of Wisconsin-Madison (1986) (UWFDM-674).

4.12 S. Jardin, Princeton Plasma Physics Laboratory, private communication (September 16, 1987). 
4.13 T.J. Dolan, Fusion Research: Principles, Experiments, and Technology (Pergamon Press, New York, 1982) 625.

4.14 F.F. Chen, Introduction to Plasma Physics and Controlled Fusion (Plenum Press, London, 1983) 183.

4.15 W.C Turner et al., "Investigation of the Magnetic Structure and the Decay of a Plasma-Gun-Generated Compact Torus," Physics of Fluids 26 (7) (1983).

4.16 B.J. Merrill, ]daho National Engineering Laboratory, EG\&G, private communication (1987).

4.17 Metals Handbook, 8th Edition, Vol. 1, Properties and Selections of Metals (American Socjety for Metals, New York 1961).

4.18 R. Hultgren et al., Selected Values of Thermodynamic Properties of Metal and Alloys (John Wiley and Sons, New York, Inc, 1963).

4.19 H. Nakamura et al., "First Wall Erosion During Plasma Disruption in Tokamak," Japan Atomic Energy Research Institute, JAERI-M 83-058 (March 1983). 


\section{Chapter 5}

\section{Parametric Analysis}

\subsection{Choice of Parameters}

The purpose of any parametric analysis is to reach useful conclusions about a specific physical problem. Such conclusions can be successfully made through the judicious selection of parameters and their organized evaluation. For this analysis, the ultimate purpose is to recommend the best material for the divertor of an experimental tokamak reactor from a disruption point of view. Specifically, this analysis is for the TIBER II design study. Past design considerations for this and similar studies have mainly focused on steady-state scenarios, which have a dramatically lower energy deposition occurring on the divertor, as compared with disruption conditions. Most of the disruption analysis previously conducted assumed peaking factors for the energ: deposition and treated the problem in only one dimension. This analysis attempts to model the phenomenon with a quasi-two-dimensional approach and to make realistic calculations for the peaking factors and global thermal effects. 
Since disruption control is poorly understood, the divertor will be ex. pected to withstand hundreds, if not thousands, of disruptions during the lifetime of the reactor. Even if the design uses replaceable divertor parts, a component with a maximum expected lifetime is still the preferred design. The longer the lifetime of an internal component, the shorter the maintenance time for the reactor. Less downtime for maintenance means higher availability for experimentation or, in a commercial reactor, for power production.

The lifetime of the divertor for a particular material is basically de. termined by the average surface ablation per disruption, the plate's original thickness, and the anticipated disruption frequency. Although the phenomenon of intense energy deposition js complex, once an average vaporization depth per disruption is determined, the lifetime of the component can be reasonably estimated. Therefore, the vaporization rate is the key thermal response indicator that requires investigation. Once the vaporization rate is determined, the ablation depth and total volume or mass ablated per disruption can be calculated. Since uniform thickness is not necessarily a design requi:cment for the divertor. the spatial dependence of these rariables is also investigated. From these spatial distributions, peaking factors can be determined. Peaking factors represent the ratio of the maximum expected value to the average expected value for a particular thermal response. With these peaking factors, a minimusn lifetime of the divertur plates can be estirnated. Also, from these spatial distributions divertor plates rould be designed with non-uniform thickness. Other aspects 
of thermal response that are considered in this thesis are power densities and surface temperatures. The surface temperature is important since the rapor pressure of the material is functionally dependent on its magnitude.

Many parameters could be evaluated by TADDPAK. This study focuses on four of primary importance. First, the material choice for the divertor is investigated. Since more than one material is suitable for steady-state operations, investigating each material from a disruption viexpoint will aid making the ultimate material choice for the divertor. Second, the pulse duration is evaluated. As with the pulse shape, a benchmark duration of 0.1 milliseconds is used for most of the study (as explained in Section 2.3). Third is the disruption pulse shape. Although a DSTAR-generated pulse shape (exponential in form) is assumed for most of this study, the dependence of a standard square pulse shape for the same total energy is considered. Last, the characteristic decay length of the plasma scrape-off layer (SOL) is investigated. Since this parameter could vary widely from assumed values, the eflects of varying its magnitude are considered.

\subsection{Material Choice}

Three candidate materials, recommended by previous steady-state analysis, are investigated in this study: graphite, tungsten, and beryllium. Figures are presented depicting the thermal response of the lower, inner, and outer divertor plates for each of these materials. Graphite is the first material presented in 
Figures 5.1 through 5.9. The data presented in these figures are based on an assumed characteristic radial decay length of $3.0 \mathrm{~cm}$ in the SOL. Figures $5.1-5.3$ illustrate the power and energy densities for the inner, lower, and outer divertor plates, respectively. The power density is represented as a surface of time and position on each plate, while the energy density shows the integration of this surface nver time. These figures also list the total energy deposited on each plate, which is the integration of the e $\mathrm{I}_{2} \mathrm{ergy}$ density curve. The corresponding figures for tungsten and beryllium are omitted since the information depicted on them is independent of material, and would therefore be redundant.

A comparison of Figures 5.1, 5.2, and 5.3 shows that the power density is a nonlinear function of position for each of the plates. This nonlinearity occurs for two reasons. First, the disruption particle fux exponentjally decays outside the separatsix. This decay prevents a linear distribution of particies on the magnetic Rux surfaces. Second. the magnetic flux surfaces, on which the charge particles move, intersect each divertor plate in an irregular manner and with varied concentration. The closer the flux surfares are and the more perpendicular their intersection with each plate's surface, the higher the power density. The intersection of the magnetic flux surfaces with the plate's surface was illustrated in Figure 2.1. As can be interpreted from this figure, each plate's position and shape within the magnetic field play a significant part in determining the energy and puwes densities it is subjected to during a disruption. Therefore, TALDPAK could be used as a design tool for evaluating wrious shapes and 


\section{INNER DIVERTOR PLATE}

MRTER]AL: Grophite

E-FOLD: $\quad 3.0 \mathrm{CM}$

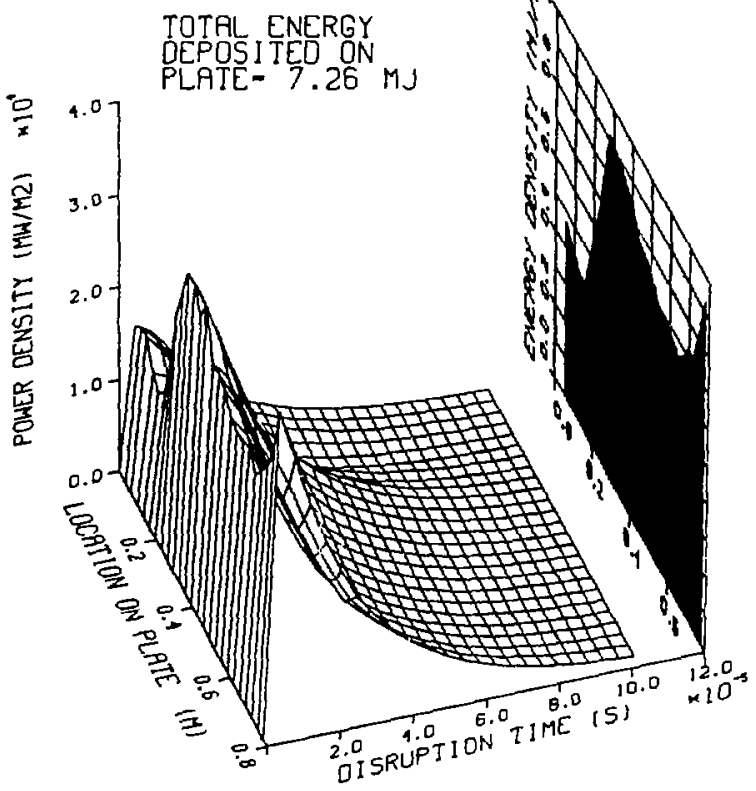

Figure 5.1: Disruption effects $(0.1 \mathrm{~ms})$ on inner divertor plate for graphite divertor material showing distribution of power/energy densities. 


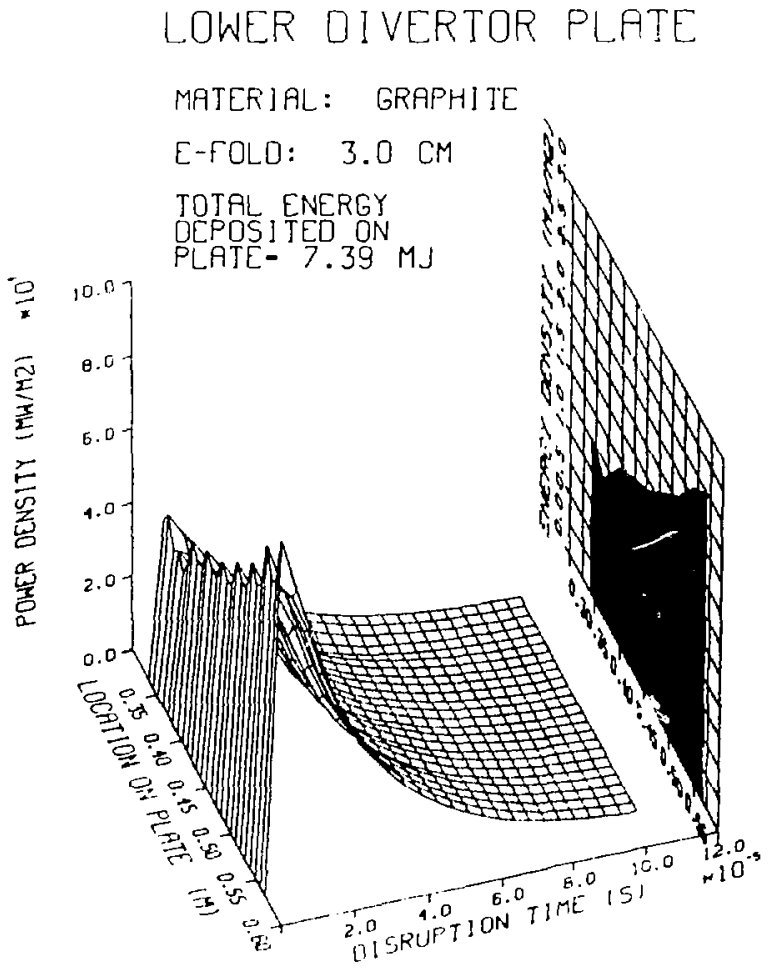

Figure 5.2: Jisruption effects $(0.1$ mas) on lower divertor plate for graphite divertor materiat showing distritution of puwer/energy densities. 


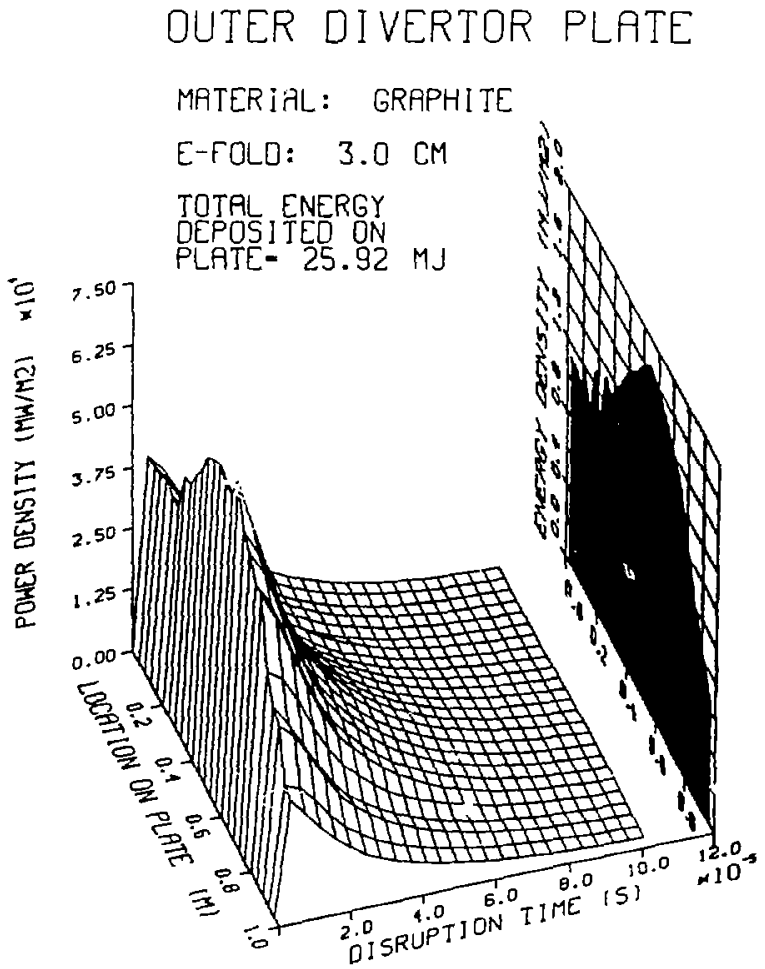

Figure 5.3: Disruption effects $(0.1 \mathrm{~ms})$ on outer divertor plate for graphite divertor material showing distribution of power/energy densities. 
positions of the plates within the racuum ressel.

As shown in Figure 5.2, the lower plate has the highest power and energy densities, $1.0 \times 10^{5} \mathrm{MW} / \mathrm{m}^{2}$ and $2.5 \mathrm{MJ} / \mathrm{m}^{2}$, respectively. This fact can be understood from the above argurnent that the higher the field-line concentration and the more perpendicular the field lines are to the plate's surface, the higher the power and energy densities. However, the lower plate does not have the most energy dcposited on its surface. The total energy deposited on each plate is determined by the distribution of energy density, the plate's total surface area, and the assumed partitioning of disruption particle flux between the inner and outer SOLs. Although the outer plate's energy density is less than that of the lower plate, its surface area is much larger; therefore, the outer plate has approximately 3.5 times the energy deposited on its surface. Another important observation about Figures 5.1, 5.2, and 5.3 is that, as a function of time, all three figures have the same exponential form. Their similar forms are expected since they are all functions of the same input function of power flow, which was shown in Figure 2.2. The significant results of these three figures are summarized in Table 5.1. The other point worth noting is that the total energy deposited on all threr plates for half the machine is greater than the assurned value of $70 \%$ of half the total stored thermal energ!: Thts apparent discrepancy is due to the assumption 'hat the remaining $30 \%$ of stored thermal energy is isotropically radjated.

The temperature distributions for the inner, fower, anc onter graphite 
Table 5.1: Summary of disruption power and energy densities on graphite divertor plates subjected to 0.1 -ms discuption.

\begin{tabular}{||l|r|r|r||}
\hline & \multicolumn{3}{|c||}{ Divertor Plate } \\
\cline { 2 - 4 } & Inner & Lower & Outer \\
\hline Maximum Power Density $\left(\mathrm{MW} / \mathrm{m}^{2}\right)$ & $3.7 \times 10^{4}$ & $1.0 \times 10^{5}$ & $7.3 \times 10^{4}$ \\
Maximum Energy Density $\left(\mathrm{MJ} / \mathrm{m}^{2}\right)$ & 0.93 & 2.5 & 1.85 \\
Peaking Factor for Energy Density & 1.4 & 1.4 & 1.4 \\
Total Energy Deposited on Plate (MJ) & 7.3 & 7.4 & 25.9 \\
\hline
\end{tabular}

plates are presented in Figures 5.4, 5.5, and 5.6.

Their functional forms follow their corresponding power distributions. However, the time scale is four times longer then the time scale on the previous set of figuras. This increase in time scale allows the observation of the cooling process once the disruption has ended. It can be seen that the cooling process is basically exponential once the disruption has terminated. Because the initial increase in energy density is rapid and intense, the surface tt:mperatures for all three plates also rapidly increase to their maximum values. This rapid increase in temperature results because the material has a thermal inertia that prevents the intense incident heai from being conducted away from the surface instantaneously. As with power and energy density, the lower plate has the highest surface temperature of approximately $5400 \mathrm{~K}$, followed by the outer and lower plates with maximums of $4950 \mathrm{~K}$ and $5270 \mathrm{~K}$, respectively. At these high temperatures, raporization is significant.

Ablation data for the three graphite plates are shown in Figures 5.7, 5.8, and 5.9. The rapurization rate is represented as a surface of time and 


\section{INNER DIVERTOR PLATE \\ MATERIAL: GRAFHITE \\ E-FOLD: $3.0 \mathrm{~cm}$}

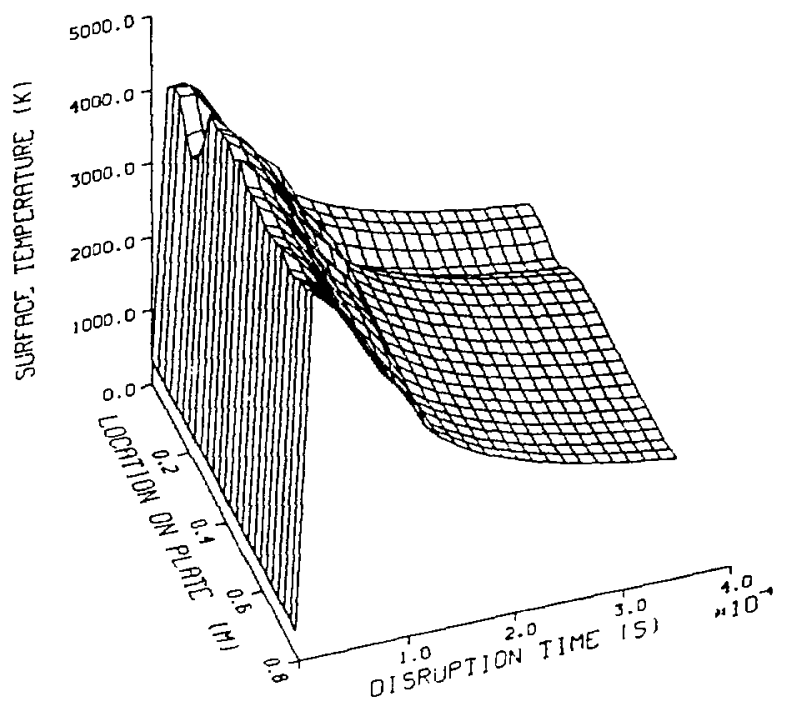

Figure 5.4: Disruption effects $(0.1 \mathrm{~ms})$ on inner divertor plate for graphite divertor material showing distribution of surface temperature. 


\section{LOWER DIVERTOR PLATE \\ MATERIAL: GRAPHITE \\ E-FOLD: $3.0 \mathrm{CM}$}

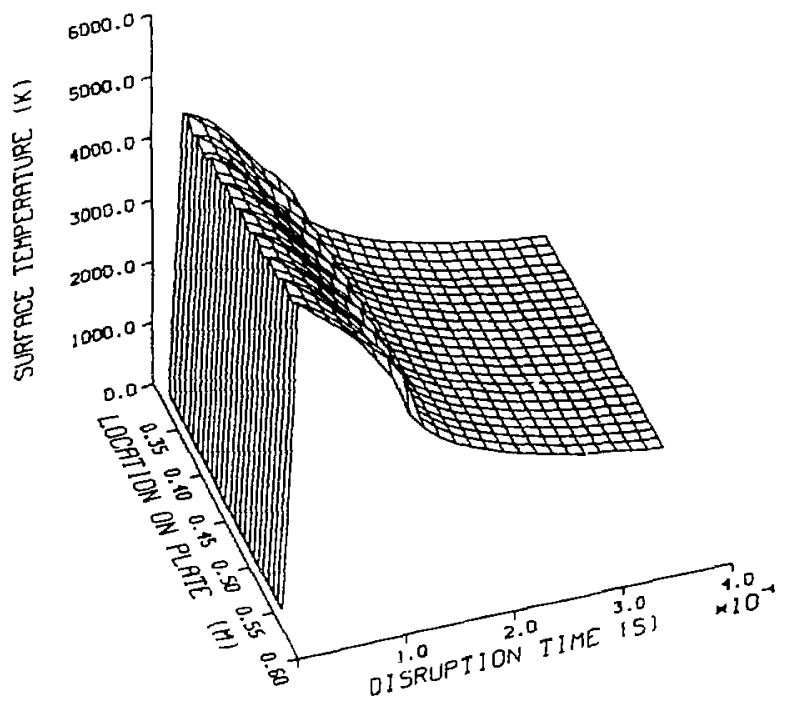

Figure 5.5: Disruption effects $(0.1 \mathrm{~ms})$ on lower divertor plate for graphite divertor material showing distribution of surface temperature. 


\section{OUTER DIVERTOR PLATE}

MATERIAL: GRAPHITE

E-FOLO: $3.0 \mathrm{cM}$

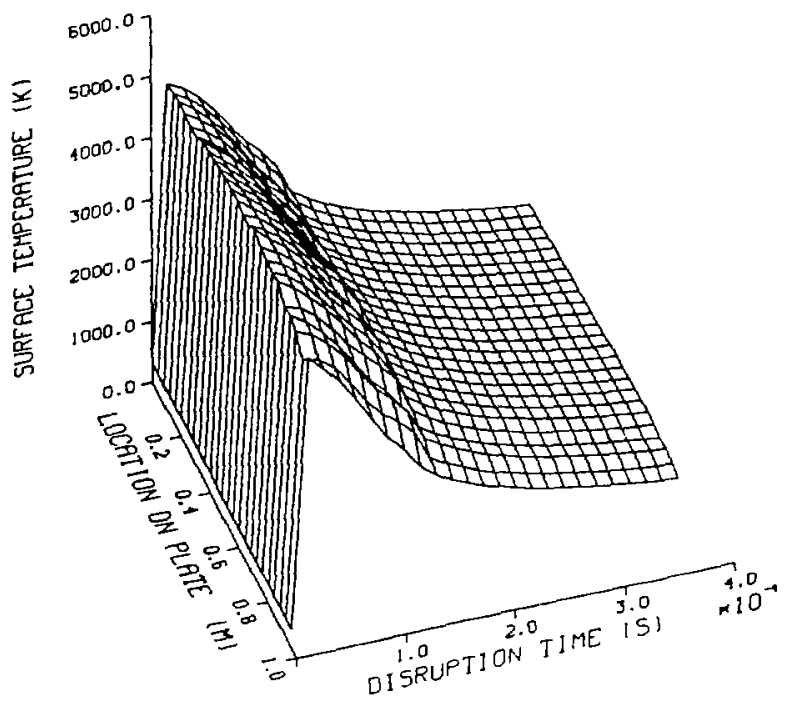

Figure 5.6: Disruption effects $(0.1$ rns) on outer divertor plate for graphite divertor material showing distribution of surface temperature. 


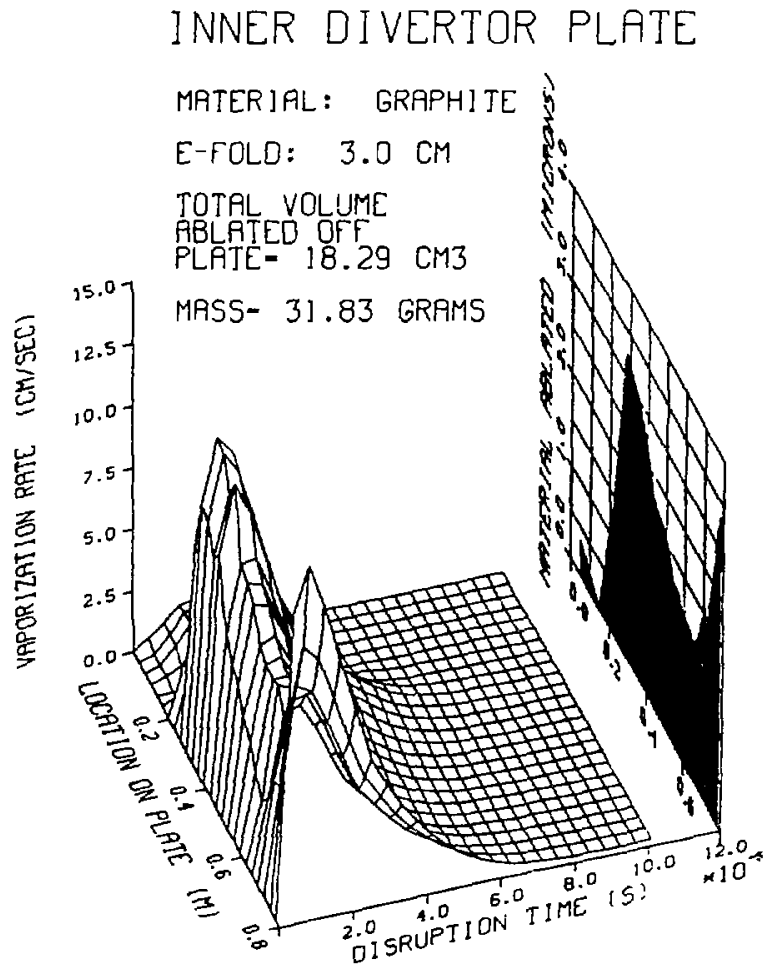

Figure 5.7: Disruption effects $(0.1 \mathrm{~ms})$ on inner divertor plate for graphite divertor material showing distribution of vaporization rate and ablation depth. 


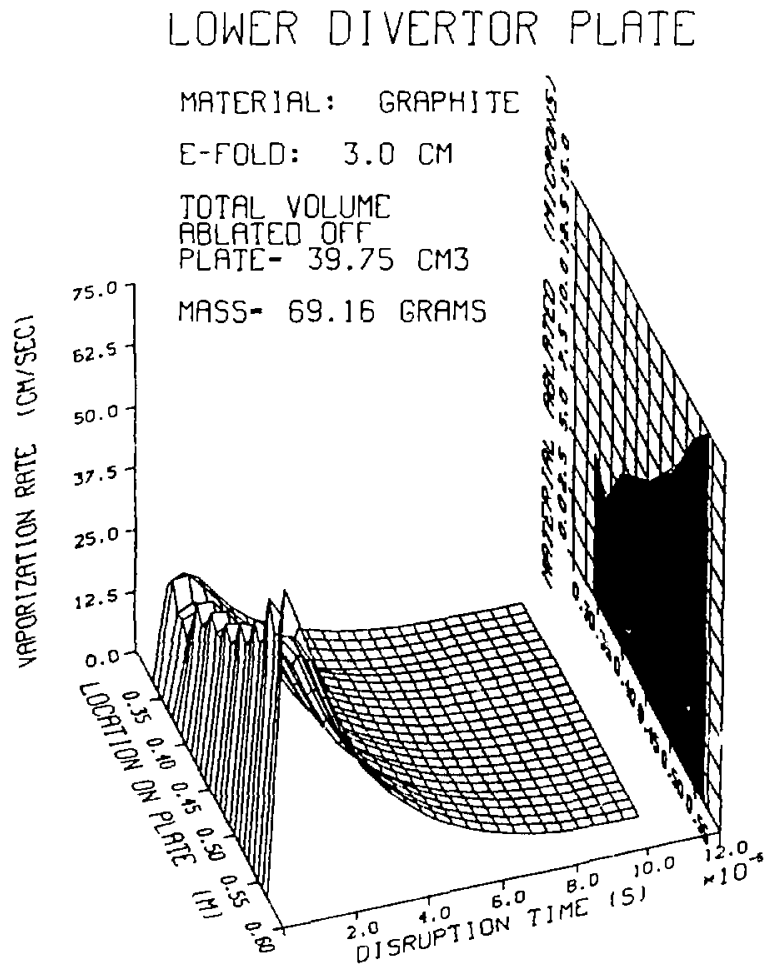

1. gure 5.8: Disruption effects $(0.1 \mathrm{~ms})$ on lower divertor plate for graphite divertor material showing distribution of vaporization rate and ablation depth. 


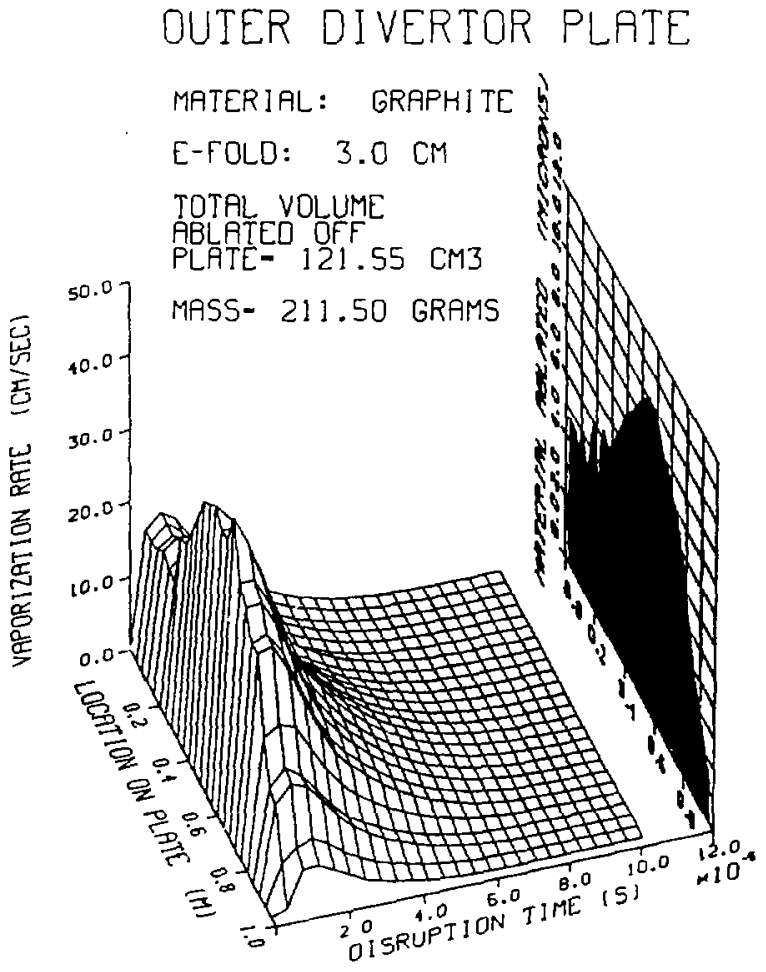

Figure 5.9: Disruption effects $(0.1 \mathrm{~ms})$ on outer divertor plate for graphite divertor material showing distribution of vaporization rate and ablation depth. 
position on each plate, while the local material ablated shows the integration of this surface over time. These figures also list the total volume and mass of the material vaporized from each plate's surface for one hemisphere of the reactor. In calculating these values, the local depth of ablation is integrated over the entire surface area of each plate. These surfaces are similar in form to those of the power density corresponding to each plate. However, there are subtle differences, which should be noted. First, the peaking factors for these figures are significantly larger. This difference in magnitude can be explained by considering the corresponding tempesature distributions and the evaporation physics at the surface of the plate. Equation 3.12 shows that the evaporation flux is proportional to the saturation vapor pressure. Since the saturation vapor pressure is an exponential function of te...perature within the temperature range considered, slight variations in the temperature can cause large variations in the evaporation flux. This phenomenon can be seen by comparing Figures 5.1 and 5.7 for the inner plate from 0.0 to 0.2 meters and Figures 5.4 and 5.7 for the same plate from 0.3 to 0.6 meters. The second comparison is the most pronounced since it shows the temperature being fairly constant as a function of position while the raporization rate dips dramatically.

The second difference is the broadening of the vapurization rate surfaces as a function of time in comparison to the power density surfaces. Again, this difference can be understood by considering the corresponding temperature distributions. Since cooling is not an instantanecous process, the temperature 
Table 5.2: Summary of ablation effects on graphite divertor plates subjected to 0.1 -ms disruption.

\begin{tabular}{||l|r|r|r||}
\hline & \multicolumn{3}{|c|}{ Divertor Plate } \\
\cline { 2 - 4 } Ablation Effect & Inner & Lower & Outer \\
\hline Maximum Vaporization Rate $(\mathrm{cm} / \mathrm{s})$ & 14.8 & 62.9 & 42.7 \\
Maximum Ablation Depth (microns) & 3.4 & 15.0 & 10.0 \\
Peaking Factor for Ablation Depth & 2.0 & 1.6 & 1.6 \\
Volume Ablated off Plate $\left(\mathrm{cm}^{3}\right)$ & 18.3 & 39.8 & 122.6 \\
Mass Ablated off Plate $(\mathrm{g})$ & 31.8 & 69.2 & 211.5 \\
\hline
\end{tabular}

lags behind the decreasing power density. In fact, it even remains high for a short period of time after the disruption energy flux has ender. Therefore, as long as the temperature is high enough, vaporization can continue at the surface of the plate. Thus, the thermal inertia causes the broadening observed in these figures.

As with power density and temperature, the lower plate has the highest vaporization rate, approximately $63 \mathrm{~cm} / \mathrm{s}$. This rate results from the maximum temperature of $5400 \mathrm{~K}$. The hot spot on the lower plate suffers 15 microns of ablation from the disruption. The ablation effects for all three graphite plates are surnmarized in Table 5.2.

Similar sets of distributions for temperature and ablation effects are presented in Figures 5.10 through 5.15 and Figures 5.16 through 5.21 for tungsten and beryllium, respectively. As with the graphite distributions, these distributior. a are for a $0.1-$ ms disruption, a $3.0-\mathrm{cm}$ characteristic radial decay length in the SOL, and the same input disruption power flux. Through the comparison of these two sets of figures and those for graphite, a recommended material 


\section{INNER DIVERTOR PLATE}

MATERIAL: TUNGSTEN

\section{E-FOLD: $\quad 3.0 \mathrm{cM}$}

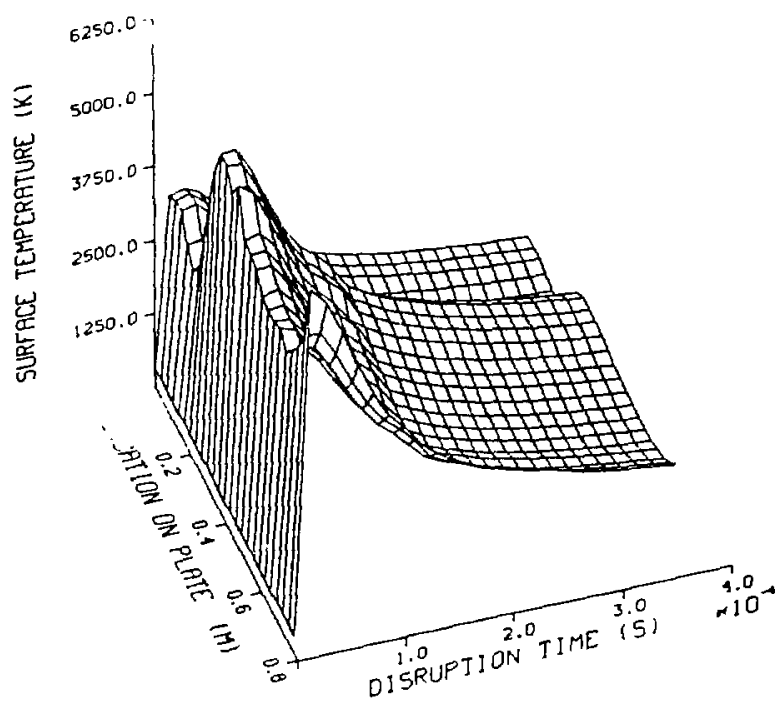

Figure 5.10: Disruption effects $(0.1 \mathrm{~ms})$ on inner divertor plate for tungsten divertor material showing distribution of surface temperature. 


\section{LOWER DIVERTOR PLATE}

\section{MATERIRL: TUNGSTEN \\ E-TOLD: $3.0 \mathrm{cM}$}

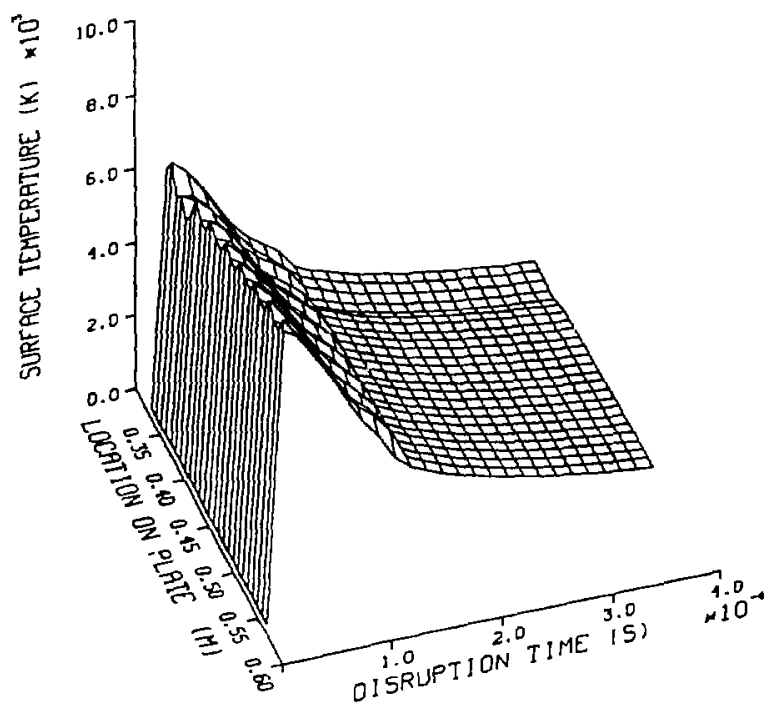

Figure 5.11: Disruption effects (0.1 ms) on lower divertor plate for tungsten divertor material showing distribution of surface temperature. 


\section{OUTER DIVERTOR PLATE}

MATERIFL: TUNGSTEN

E-FOLD: $\quad 3.0 \mathrm{CM}$

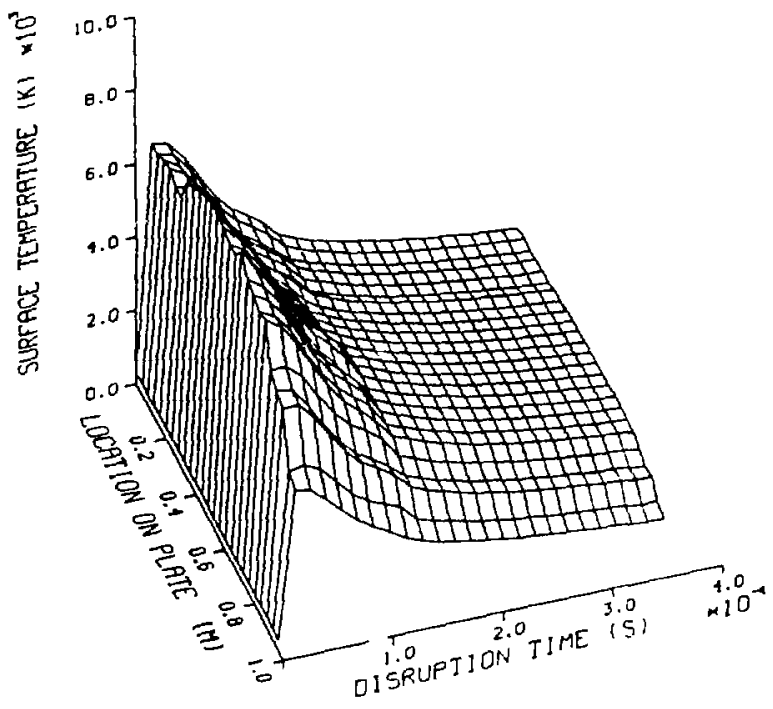

Figure 5.12: Disruption effects $(0.1 \mathrm{~ms})$ on outer divertor plate for tungsten divertor material showing distribution of surface temperature. 


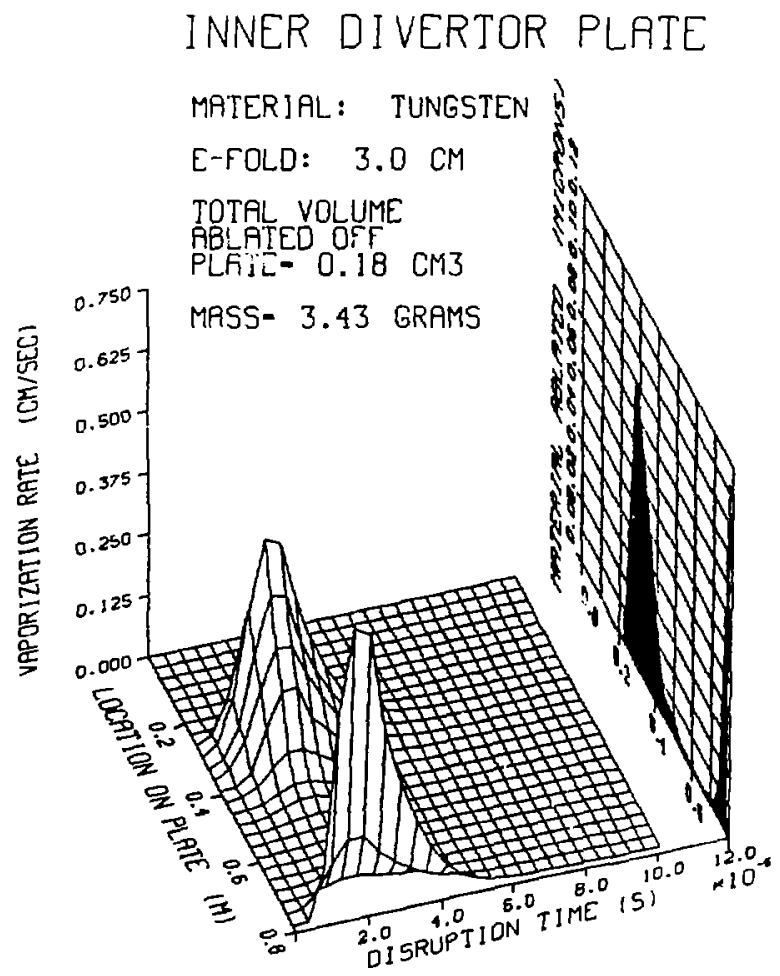

Figure 5.13: Disruption effects $(0.1 \mathrm{~ms})$ on inner divertor plate for tungsten divertor material showing distribution of vaporization rate and ablation depth. 


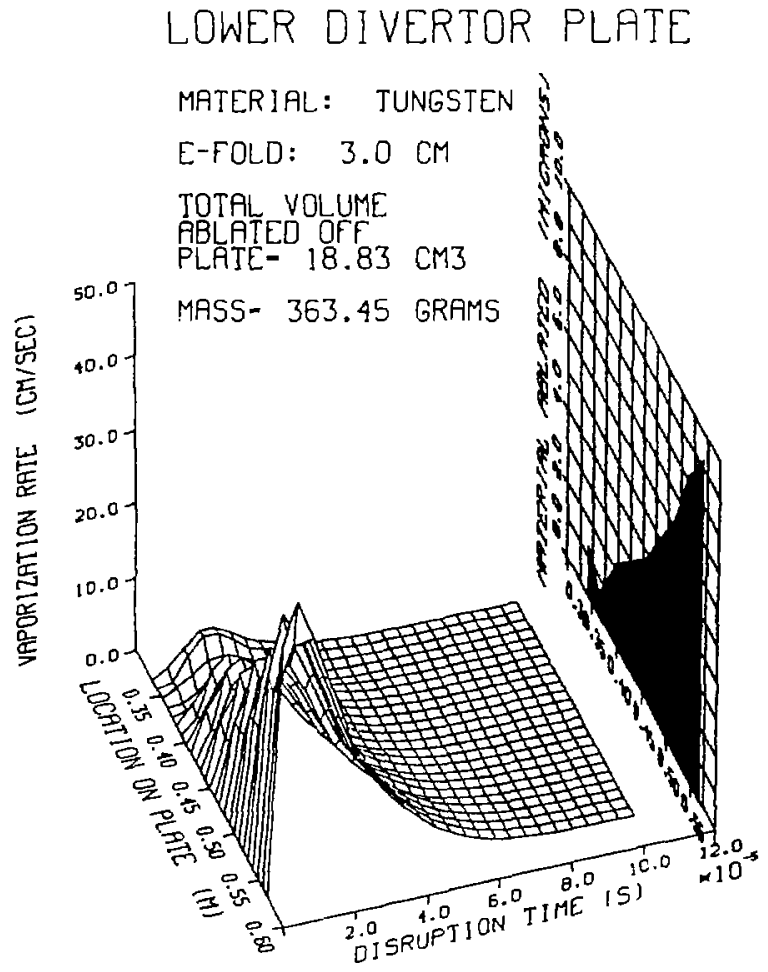

Figure 5.14: Disruption effects $(0.1 \mathrm{~ms})$ on lower divertor plate for tungsten divertor material showing distribution of vaporization rate and ablation depth. 


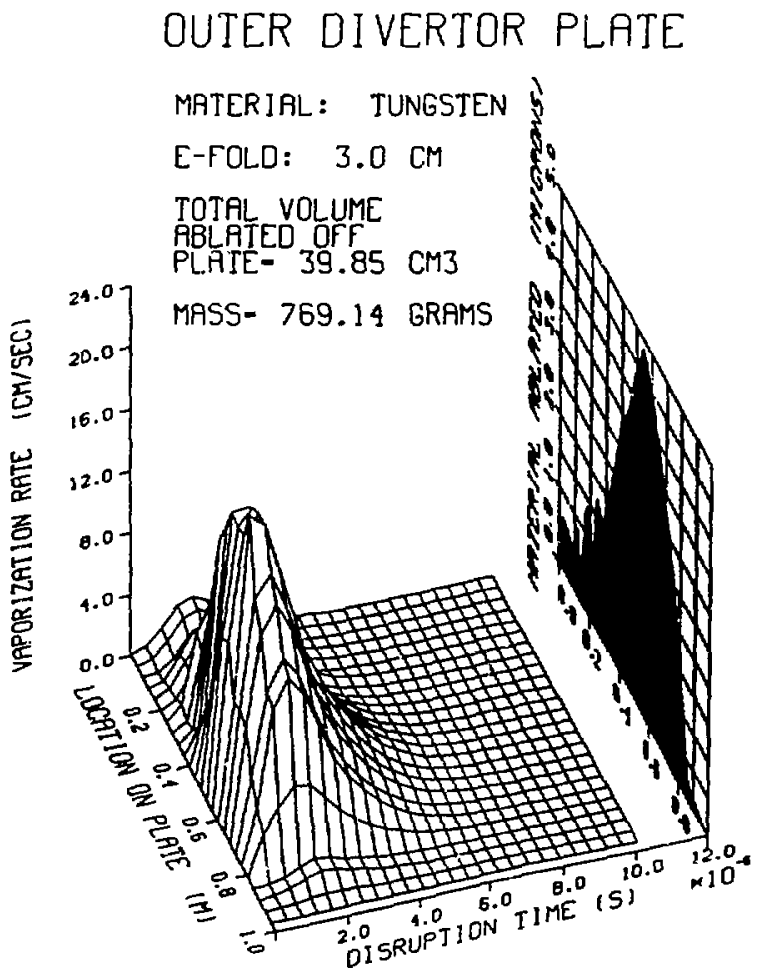

Figure 5.15: Disruption effects $(0.1 \mathrm{~ms})$ on outer divertor plate for tungster divertor material showing distribution of vaporization rate and ablation depth. 
Table 5.3: Comparison of ablation effects on divertor plates of graphite, tungsten, and beryllium subjected to $0.1-\mathrm{ms}$ disription.

\begin{tabular}{|c|c|c|c|c|}
\hline \multirow[b]{2}{*}{ Plate } & \multirow[b]{2}{*}{ Ablation Effect } & \multicolumn{3}{|c|}{ Material } \\
\hline & & Graphite & Tungsten & Beryllium \\
\hline \multirow[t]{6}{*}{ Innex } & Maximum Temperature (K) & 4950 & 6202 & $356 \overline{4}$ \\
\hline & Maximum Vaporization Rate $(\mathrm{cm} / \mathrm{s})$ & 14.8 & 0.63 & 21.3 \\
\hline & Maximum Ablation Depth (microns) & 3.4 & 0.11 & 5.1 \\
\hline & Peaking Factor for Ablation Depth & 2.0 & 6.7 & 2.1 \\
\hline & Volume Ablated off Plate $\left(\mathrm{cm}^{3}\right)$ & 18.3 & 0.18 & 26.3 \\
\hline & Mass Ablated off Plate (g) & 31.8 & 3.4 & 48.7 \\
\hline \multirow[t]{6}{*}{ Lower } & Maximum Temperature (K) & 5400 & $8 \overline{8650}$ & 4251 \\
\hline & Maximum Vaporization Rate $(\mathrm{cm} / \mathrm{s})$ & 14.8 & 40.2 & 101.5 \\
\hline & Maximum Ablation Depih (microns) & 15.0 & 9.1 & 24.4 \\
\hline & Peaking Factor for Ablation Depth & 1.6 & 2.1 & 1.6 \\
\hline & Volume Ablated off Plate $\left(\mathrm{cm}^{3}\right)$ & 39.8 & i8.8 & 64.5 \\
\hline & Mass Ablated off Plate $(\mathrm{g})$ & 69.2 & 363.4 & 119.4 \\
\hline \multirow[t]{6}{*}{ Outer } & Maximum Temperature (K) & 5270 & 8170 & 4052 \\
\hline & Maximum Vaporization Rate $(\mathrm{cm} / \mathrm{s})$ & 14.8 & 21.8 & 68.2 \\
\hline & Maximum Ablation Depth (microns) & 10.0 & 4.8 & 16.4 \\
\hline & Peaking Factor for Ablation Depth & 1.6 & 2.4 & 1.6 \\
\hline & Volume Ablated of Plate $\left(\mathrm{cm}^{3}\right)$ & 121.6 & 39.8 & 194.8 \\
\hline & Mass Ablated off Plate (g) & 211.5 & 769.1 & 360.4 \\
\hline \multirow[t]{2}{*}{ ALL } & Volume Ablated $\left(\mathrm{cm}^{3}\right)$ & 361 & 118 & 571 \\
\hline & Mass Ablated (g) & 625 & 2272 & 1057 \\
\hline
\end{tabular}

choice for disruption conditions will be made. The ablation effects for the three materials are presented in Table 5.3. As shown in this table, the highest surface temperatures occur with tungsten. This difference is essentially true because of the functional dependence of tungsten's vapor pressure on temperature. From Equation 4.5 and its corresponding coefficients in Table 4.3, it can be seen that the rapor pressure for tungsten is lower compared to graphite and 


\section{INNER DIVERTOR PLATE}

MATER]AL: BERYLLIUTI

E-FOLO: $3.0 \mathrm{cM}$

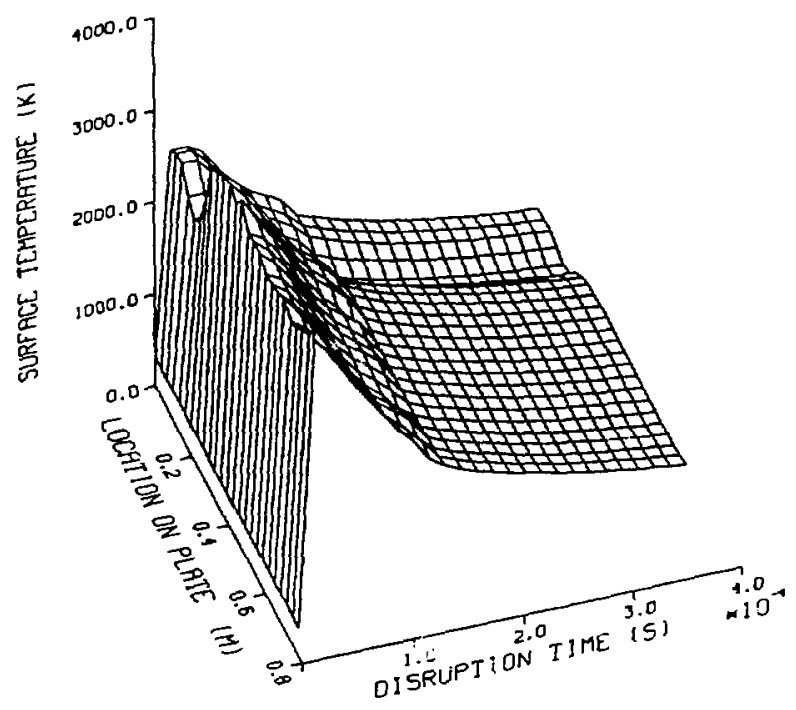

Figure 5.16: Disruption effects $(0.1 \mathrm{~ms})$ on inner divertor plate for beryllium divertor material showing distribution of surface temperature. 


\section{LOWER DIVERTOR PLATE}

\section{MATERIFL: BERYLLIUM}

\section{E-FOLD: $\quad 3.0 \mathrm{CM}$}

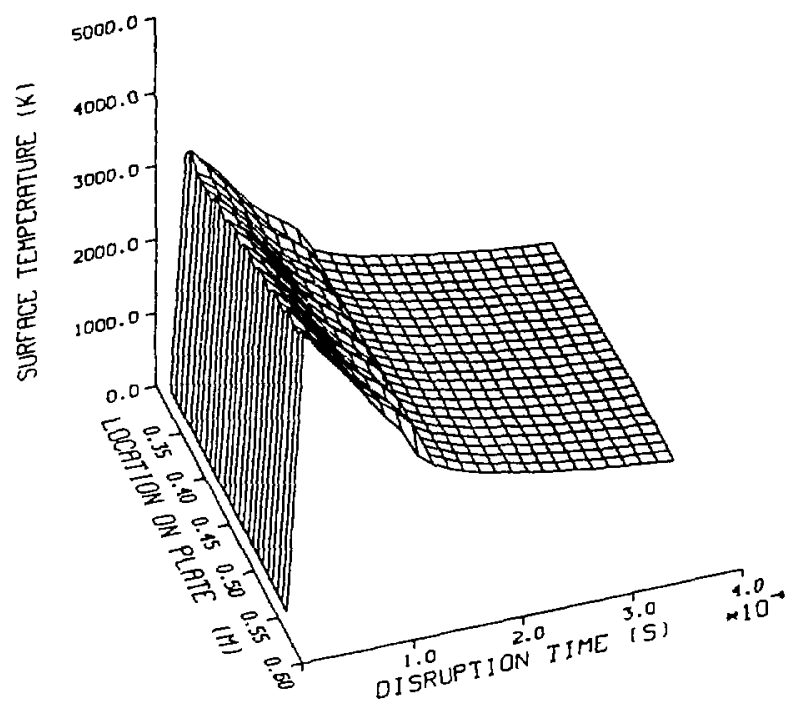

Figure 5.17: Disruption effects (0.1 ms) on lower dives tor plate for beryllium divertor material snowing distribution of surface temperature. 


\section{OUTER DIVERTOR PLATE \\ MATERIAL: BERYLLIUM \\ E-FOLD: $3.0 \mathrm{CM}$}

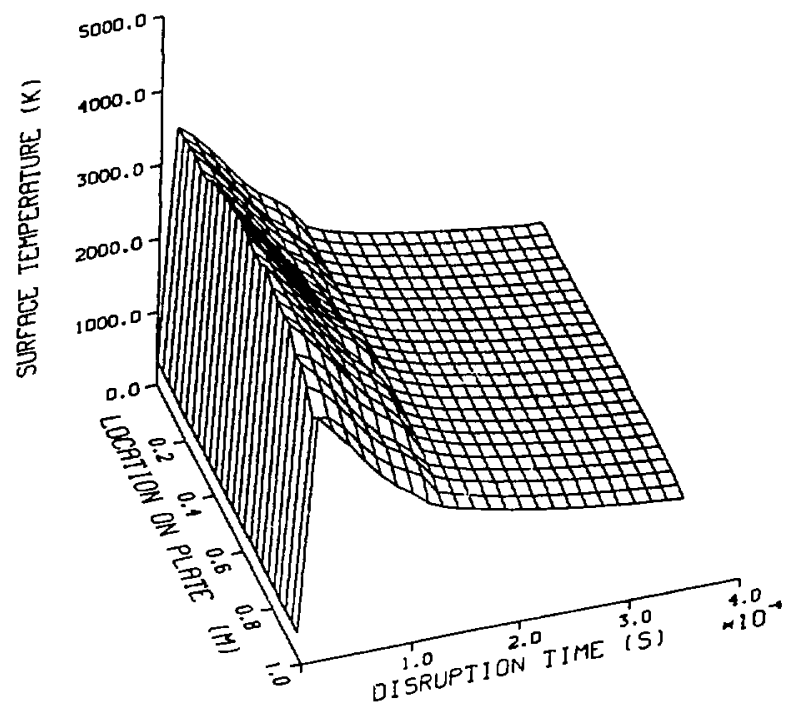

Figure 5.18: Disruption effects $(0.1 \mathrm{~ms})$ on outer divertor plate for beryllium divertor material showing distribution of surface temperature. 


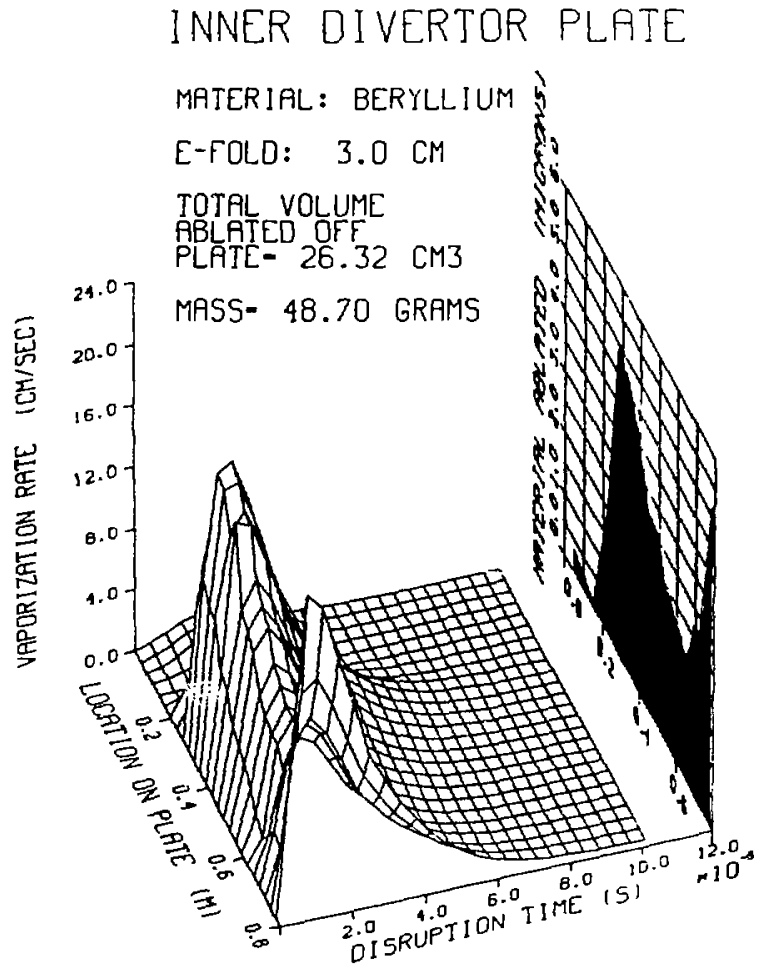

Figure 5.19: Disruption effects $(0.1 \mathrm{~ms})$ on inner divertor plate for beryllium divertor material showing distribution of vaporization rate and ablation depth. 


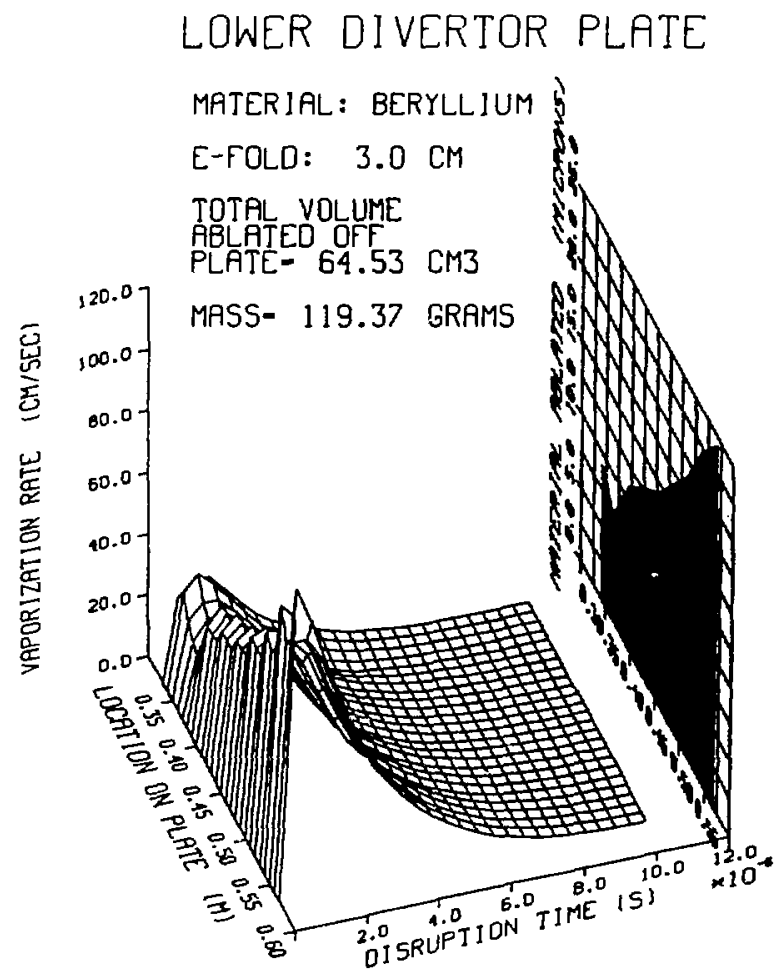

Figure 5.20: Disruption effects $(0.1 \mathrm{~ms})$ on lownr divertor plate for beryllium divertor material showing distribution of vaposization rate and ablation depth. 


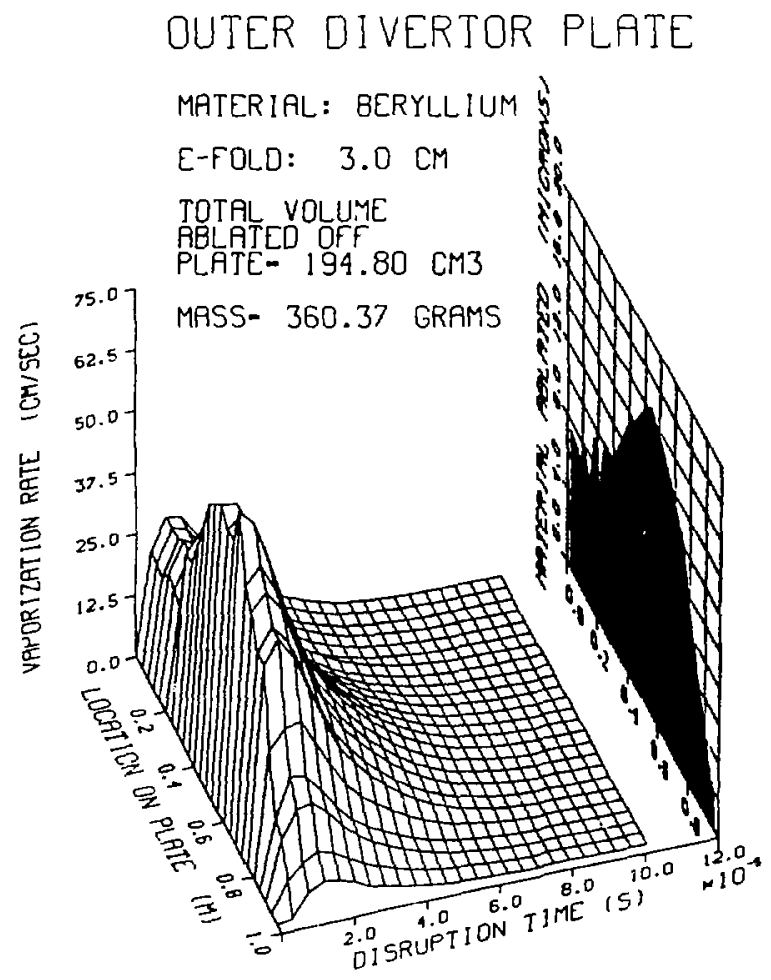

Figure 5.21: Disruption effects $(0.1 \mathrm{~ms})$ on outer divertor plate for beryllium divertor material showing distribution of raporization rate and ablation depth. 
beryllium for the same temperature. Therefore, tungsten must reach a higher temperature to have the same amount of energy consumed by vaporization. This requirement for a higher temperature is also true because the heat of vaporization for tungsten is lower than for the other two materials (see Table 4.2). Again, only through a larger vaporization flux will enough energy be consumed through vaporization to conserve energy on the surface of the plate.

The high surface temperatures calculated for tungsten may invalidate the method of solution for this problem. The main concern is that the critical temperature for tungsten may have been reached. If this is true, then no transition between the liquid and gas phase will occur. This lack of transition means the latent heat of fusion employed in the calculations of this model is no longer appropriate for this high temperature region. Since actual experimental data does not exist for the critical temperature of tungsten and theoretical conjecture for its value is limited, it is assumed that the high temperatures calculated are still less than the critical temperature of tungsten. This assumption is fairly reasonable since the corresponding pressure is approximately vacuum. However, it still requires further investigation to be considered completely valid.

Although melting data has not been presented in this analysis, the impact of melting in the lower temperature regions can be inferred from the data presented in Table 5.3. By comparing the maximum raporization rates for graphite and tungsten an inconsistency between the inner and lower plate becomes apparent. For the inner plate, graphite has the higher maximum va- 
porization rale, while for the lower plate tungsten has the higher rate. This difference can be attributed to the increase of melting that tungsten experiences at lower temperatures. As a result of more melting occurring, less energy is available to cause vaporization. At higher temperatures, vaporization dominates and negligible melting occurs.

The vaporization rate is the velocity at which the surface recedes time the liquid density. Comparing this rate for the three materials shows that the maximum value for each material corresponds to its maximum temperature. However, the relative difference in vaporization rate compared to surface temperature is not the same for the three materials. For example, tungsten has the highest surface temperature, but beryllium has the highest vaporization rate. This relative difference between the magnitudes of surface temperature and vaporization rate results from the differences in material properties such as vapor pressure, and the method of solving the moving boundary problem as described in Chapter 3. For each material, the maximum ablated depth and volume have the same relative magnitudes to the vaporization rate. This correspondence is expected since the integration of the local vaporization rate over time yiejds the total local ablated depth, and for each plate, the integration of the local ablated depth over the surface area leads to the total volume. However, the relative magnitudes in mass ablated is different due to the relative differences in material density. Although tungsten has less volume ablated than graphite and beryllium, it has more mass ablated since its density is so much higher. 
Table 5.4: Disruption erosion lifetime parameters for graphite, tungsten, and beryllium subjected to $0.1 \mathrm{~ms}$ disruption.

\begin{tabular}{||l|r|r|r||}
\hline & \multicolumn{3}{|c||}{ Material } \\
\cline { 2 - 4 } & Graphite & Tungsten & Beryllium \\
\hline Maximum Allowed Thickness (mm) & 20 & 2 & 10 \\
Maximum Depth Ablated $(\mu \mathrm{m})$ & 15.0 & 9.1 & 24.4 \\
Disruption Erosion Lifetime & 1300 & 220 & 410 \\
\hline
\end{tabular}

The above comparison provides part of the basis for choosing the best material for the divertor under a disruption scenario. To complete the reasoning for such a choice, further consideration must be given to the allowed thickness of plate material. Mechanical performance under steady-state heat loads dictates the maximum allowable thicknesses for the three materials. The therrnal stress limits for material thickness are shown in Table 5.4. These thicknesses were the accepted parameters for TIBER II and were previously calculated by Haines [5.1]. The maximum ablated depths shown in this table are for the lower divertor plate. These values were used since they represent the worst case among the three plates. The disruption erosion lifetime, also shown in this table, is the number of disruptions the plate wan be expected to survive based on its thickness and the amount of material ablated per disruption.

\subsection{Disruption Pulse Length}

Although a 0.1-milliseconds thermal quench is assumed for the calculations of Section 5.2 , it is not necessarily true that the duration of this phase of the disruption will always be this order of magnitude. Therefore, it will be worthwhile 
to evaluate the response of the divertor plates for disruption pulse lengths of different magnitudes. Since graphite appears to be the initial choice of material for the divertor plates of TIBER II [5.2], it will be the focus of this section. Figures 5.22 through 5.26 present a summary of the important thermal response parameters as a function of disruption time. The pulse lengths vary from 1 microsecond to 10 milliseconds. For convenience, each figure depicts data for all three plates.

Figure 5.22 illustrates the maximum surface temperature as a function of disruption pulse length. As with the 0.1-millisecond pulse, the lower plate has the highest surface temperatures over the entire range of pulse durations. This dominance is expected since the total local power density increases or decreases proportionately to pulse length. The most interesting feature of this figure is the change of slope, in all three curves, occurring at approximately $1.0 \mathrm{~ns}$. Prior to this pulse duration, the maximum temperature is essentially logarithmic. After this point, the curves initially increase in slope and then appear to approach a minimum temperature. This change in slope can best be understood by considering the decrease in maximum heat flux as a result of increasing the pulse length. At pulse lengths greater than $0.1 \mathrm{~ms}$, the total energy has been spread out over such a long period of time that the resulting heat flux is not larg:enough to cause any significant vasorization. From Equation 4.5, note that at temperatures below approximately $5000 \mathrm{~K}$ the vapor pressure is no longer significant for graphite. Hence, the main way for energy to leave the surface is 
by conduction. The functional form of Equation 4.5 is strongly supported in this low temperature region by experimental data. On the contrary, the validity of Equation 4.5 in the high-temperature region is somewhat in question since collecting experimental data to confirm or contradict its form is impossible with current technology. Its form only represents the best extrapolation of experimentally confirmed vapor pressure data at much lower temperatures. Since the surface temperature, vapor pressure, and vaporization rate intimately depend on each other through energy conservation, it is reasonable to assume that all three parameters will have the same functional form; and physically, the temperature and the vaporization rate must follow the vapor pressure form since it is the basic property of the material. This ussumption is reassnably confirmed by comparing Figures 5.22 and 5.23. The curves of Figure 5.23, which depict the maximum vaporization rate as a function of disruption pulse length, change slope at the same point as those of Figure 5.22. Above this point, both curves are essentially the same. However, the vaporization rate is plotted on a logarithmic scale. An obvious difference between these two sets of curves is that the vaporization rate curves in Figure 5.23 decrease to zero after the common point of slope change, while the temperature curves in Figure 5.22 approach a constant value. The functional forms of both sets of curves, in this limit, result from the disruption pulse being long enough to prevent any vaporization of or significant temperature rise in the material. $O_{i}$ in other words, the thermal response time of the material is shorter than the pulse length, so the incident 


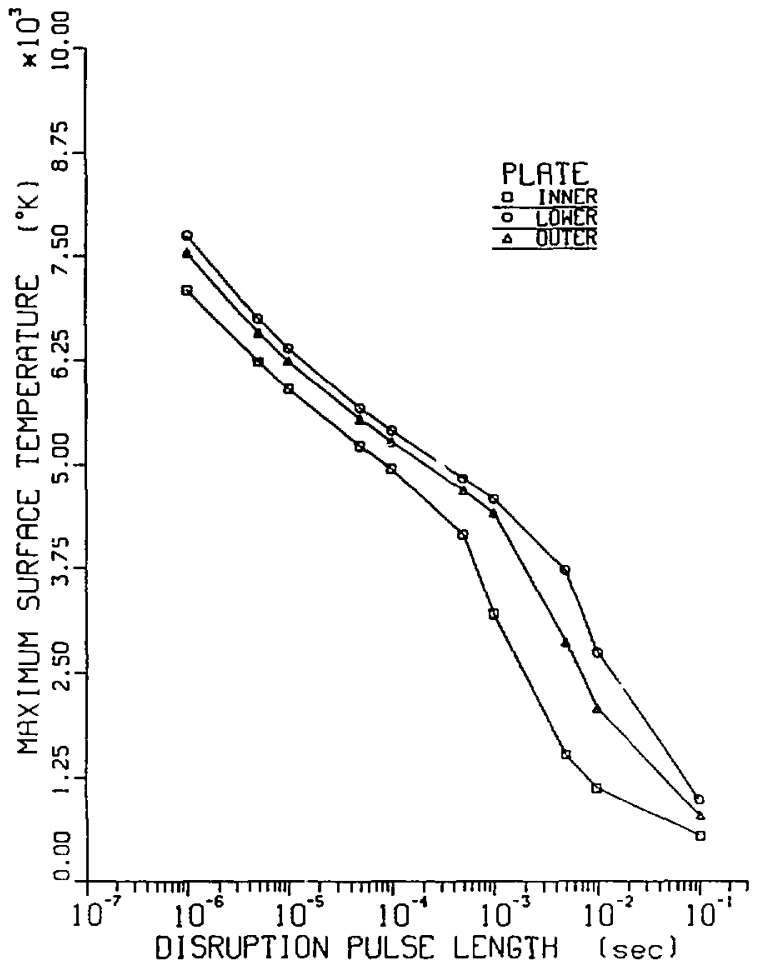

Figure 5.22. Maximum temperature on each graphite divertor plate $3 . ;$ a function of disruption pulse length. 


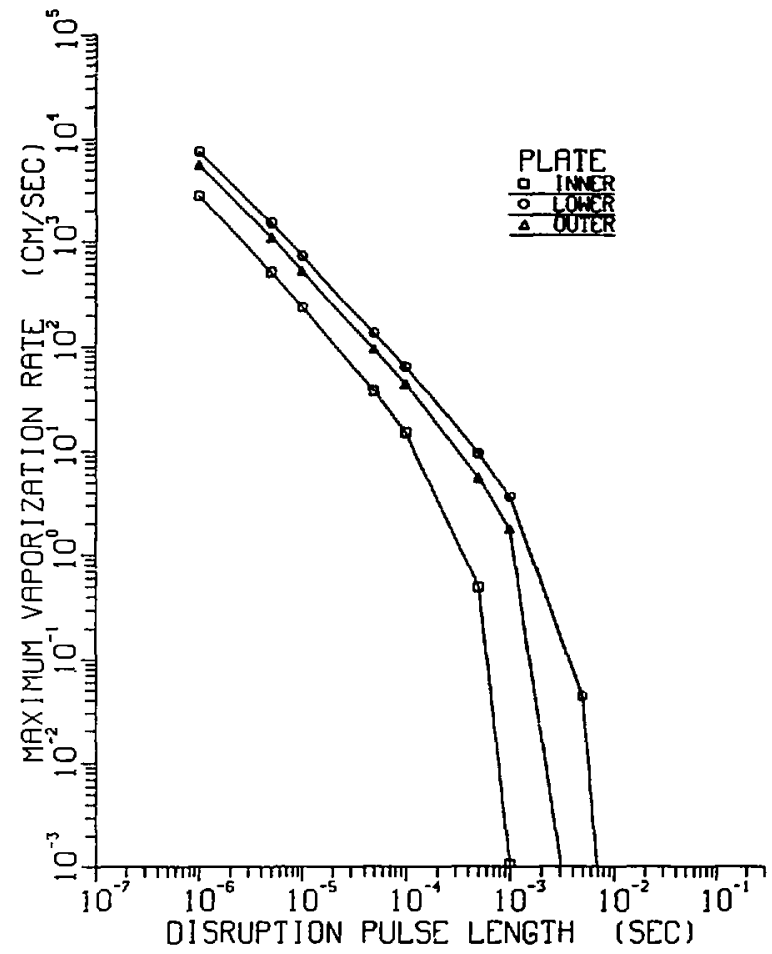

Figure 5.23: Maximum vaporization rate on each graphite divertor plate as a function of disruption pulse length. 
energy can be conducted away from the surface instead of being consumed in vaporization or accommodated by a large temperature rise.

The parameter that essentially determines the life of the divertor plate, assuming a frequency of disruptions, is the maximum vaporization depth per disruption. This parameter is presented in Figure 5.24 as a function of disruption pulse duration. Again, the lower plate has the greatest ablation depths over the entire range of pulse lengths. In addition, these curves have two noteworthy features. First, all three curves seem to approach a constant value as the disruption pulse length approaches zero. Approaching a constant depth suggests that radiation is perhaps starting to become significant in this hightemperature region. The other factor that could be, in part, responsible for this limit, is that the vaporization model used for these calculations is no longer valid in this regime. In either case, the validity of the data in this limit should not be accepted without question. Sccond, the curves in Figure 5.24 have extreme gradients over the range of pulse duration from 0.1 to $10.0 \mathrm{~ms}$. For a small change in disruption pulse duration, there is a large change in the maximum ablated depth. The largest gradient occurs on the curve for the lower plate. This sensitivity to pulse duration occurs in the most likely range of time scales for the actual thermal quench of a major disruption. Accuracy in determining the correct pulse length becomes crucial when designing components against disruptions.

Another parameter important in divertor plate design is the peaking fac- 


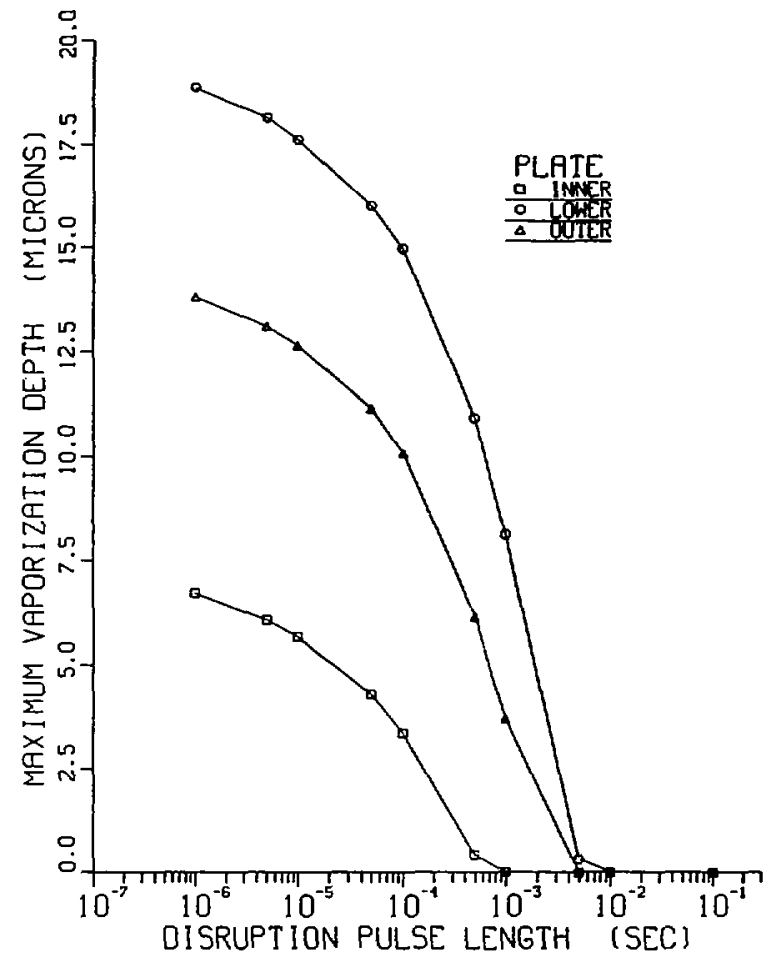

Figure 5.24: Maximum vaporization depth on each graphite divertor piate as a function of disruption pulse length. 
tors for vaporization. Knowing the values for this parameter allows the engineer use the average vaporization calculations over the surface of a divertor plate. These peaking factors are shown in Figure 5.25 as a function of disruption pulse length. The curves in this figure suggest that in the short pulse limit, the peaking factors for all three plates are a constant of approximately 1.4. However, in the long pulse limit, the sensitivity of vaporization to a critical pulse length is implied by the dramatic increase in these values. Although in this limit the vaporization will not be significant, the sharp increase implies that the vaporization has become localized to small regions on the plate. Basically, the average vaporization of the entire plate goes to zero while some localized vaporization still occurs. This localized vaporization obviously occurs at the hot spot of each plate.

The last parameter to be discussed in this section is the total volume ablated. Its functional dependence on disruption pulse duration is shown in Figure 5.26. In this figure the outer plate dominates because of the geometry and its radial dependence on surface area. Another interesting point worth discussing is the apparent convergence of the curves in this figure for the lower and inner plates. Although the models used are not valid in the limit, this convergence results from the particular configuration of the magnetic flux surfaces jntersecting each plate. Specifically, the average vaporization is higher for the inner plate for shorter pulse length. Also the total affected surface area of the inner plate is greater than that of the lower plate. 


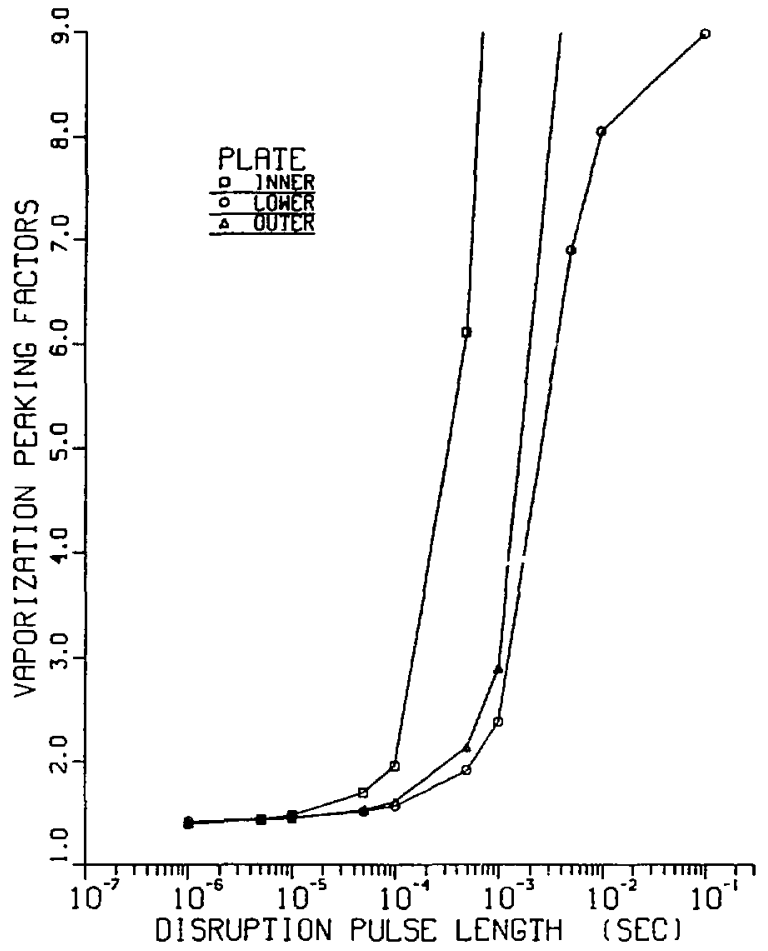

Figure 5.25: Vaporization peaking factors on each graphite divertor plate as a function of disruption pulse length. 


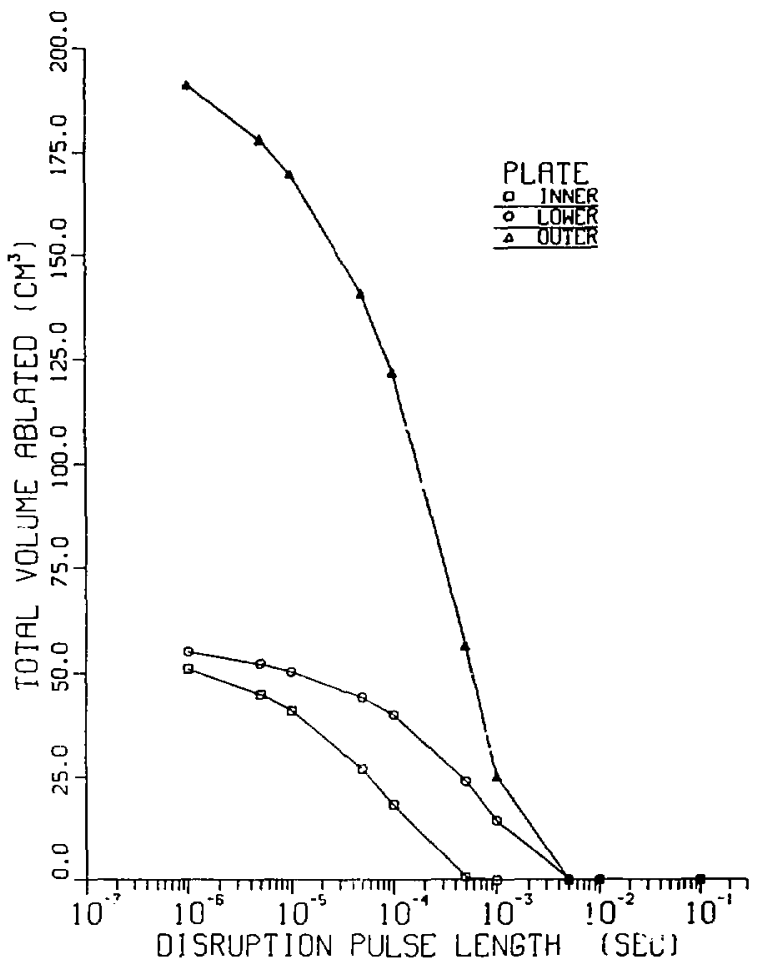

Figure 5.26: Total volume ablated from each graphite divertor plate as a functior. of disruption pulse length for one hemisphere of the machine. 


\subsection{Disruption Pulse Shape}

Since this investigation has used a disruption pulse shape similar to the form of disruptions observed experimentally and simulated mathematically, it will be valuable to consider the difference in the thermal response of the material for a square pulse shape. Such consideration is worthwhile since past analysis of one-dimensional disruption have mainly used this standard shape and not the exponential form used by this investigation. Figures 5.27 through 5.35 present the same type of information as Figures 5.1 through 5.9. The only difference is the pulse shape and the resulting thermal response. Figures 5.27 through 5.29 show the power and energy densities as a function of time and position for the inner, lower, and outer divertor plates, respectively. Although the shapes of these surfaces as a function of time are different, compared to the corresponding surfaces in Figures 5.1, 5.2, and 5.3, the total energy is essentially the same. In other words, the total energy represented by the DSTAR pulse and the square pulse is the same. The slight difference occurs due to the method of integration between the two sets of surfaces and is not a contributing factor to difference in the thermal response between the two pulse shapes. These figures show that the power density for the square pulse is 5 times smaller than the initial spike of the DSTAR profile, and by definition remains constant for the duration of the disruption pulse. The significance of this observation becomes apparent when examining the corresponding temperature profiles in Figures 5.30, 5.31, and 5.32 . 


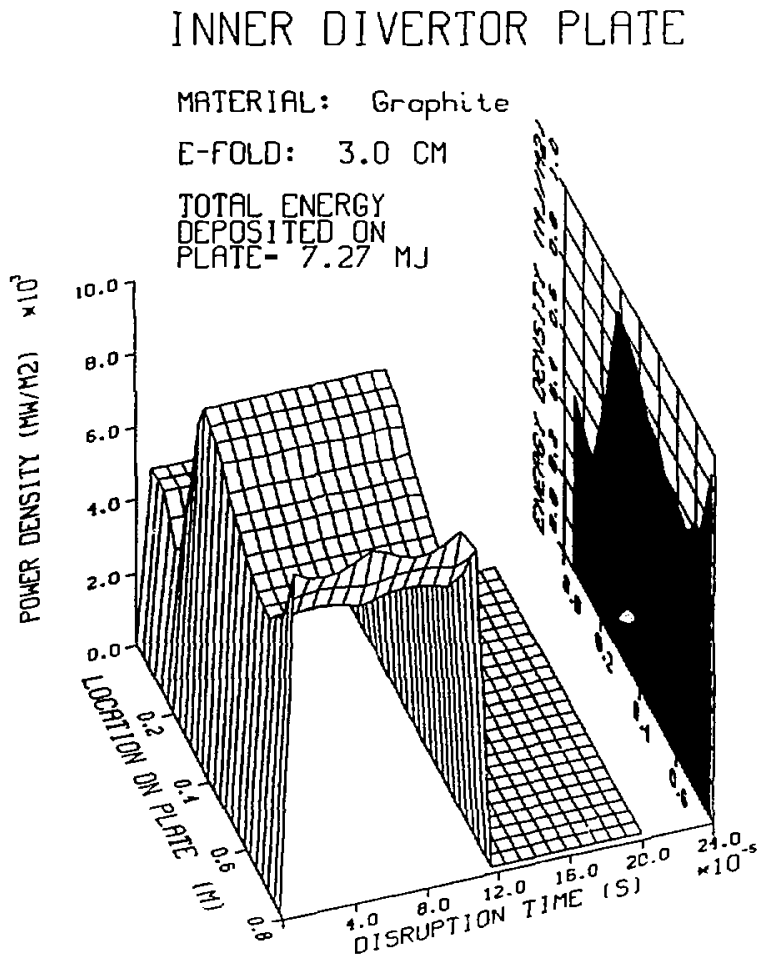

Figure 5.27: Disruption effects of $0.1 \mathrm{~ms}$ square pulse on inner divertor plate for graphite divertor material showing distribution of power and energy densities. 


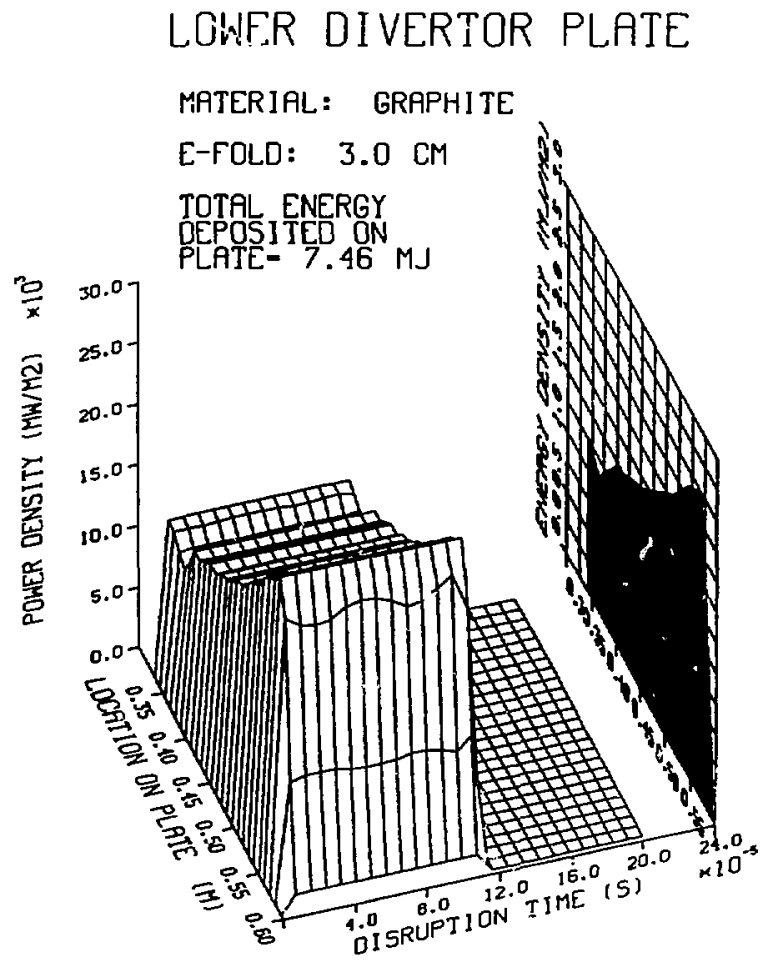

Figure 5.28: Disruption effects of $0.1 \mathrm{~ms}$ square pulse on lower divertor plate for graphite divertor material showing distribution of power and energy densities. 


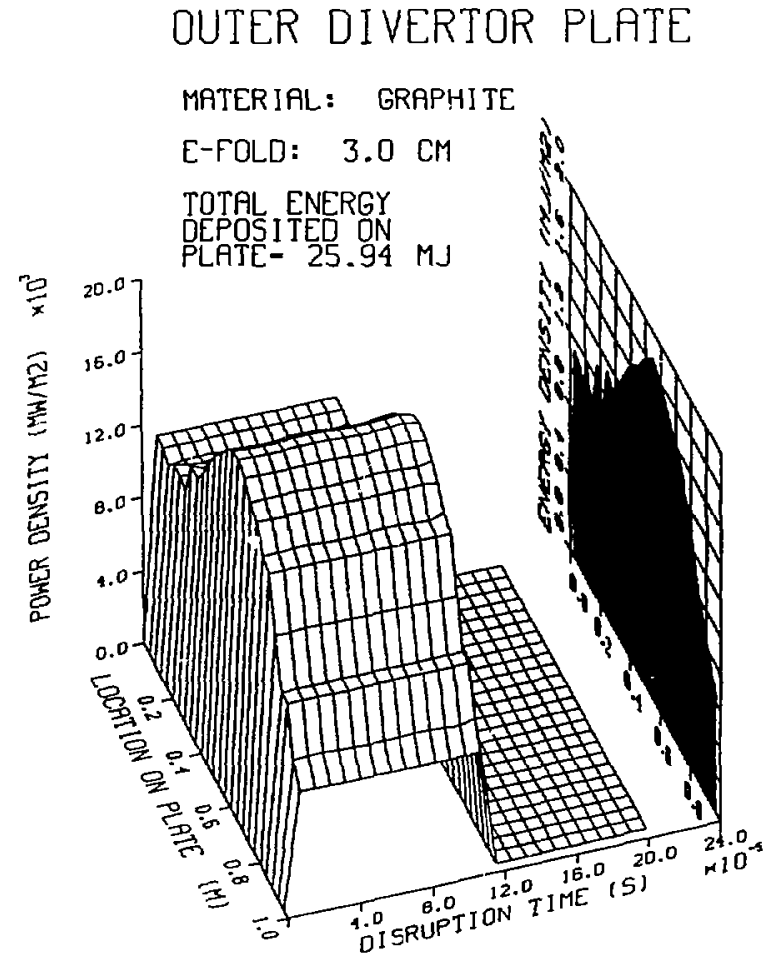

Figure 5.29: Disruption effects of $0.1 \mathrm{~ms}$ square pulse on outer divertor plate for graphite divertor material showing distribution of power and energy densities. 


\section{INNER DIVERTOR PLATE}

MATERIAL: GRAPHITE

\section{E-FOLO: $\quad 3.0 \mathrm{CM}$}

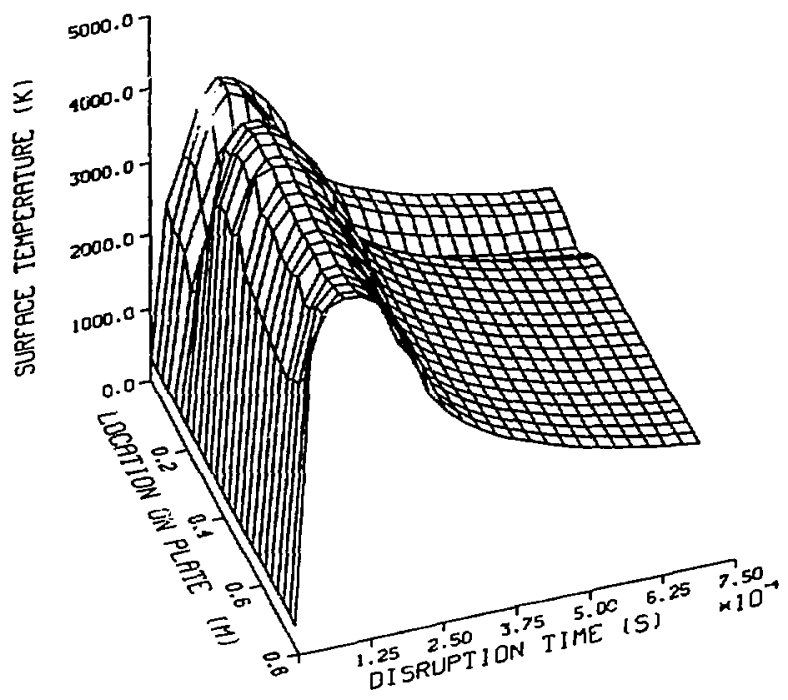

Figure 5.30: Disruption effects of $0.1 \mathrm{~ms}$ square pulse on inner divertor plate for graphite divertor material showing distribution of surface temperature. 


\section{LOWER DIVERTOR PLATE}

\section{MATERIAL : GRAPHITE}

\section{E-FOLD: $\quad 3.0 \mathrm{CM}$}

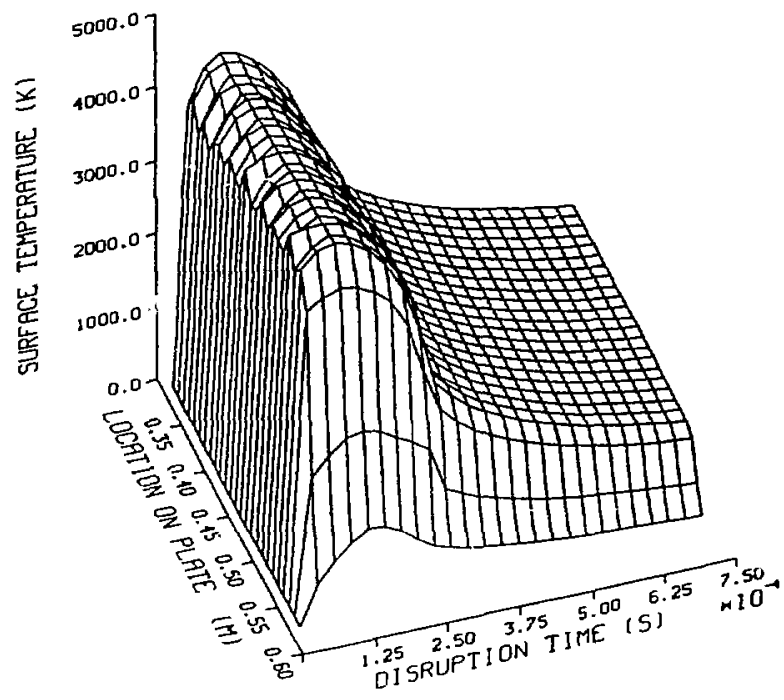

Figure 5.31: Disruption effects of $0.1 \mathrm{~ms}$ square pulse on lower divertor plate for graphite divertor material showing disiribution of surface temperature. 


\section{OUTER DIVERTOR PLATE}

\section{MATERIAL: GRAPHITE}

\section{E-FOLD: $\quad 3.0 \mathrm{CM}$}

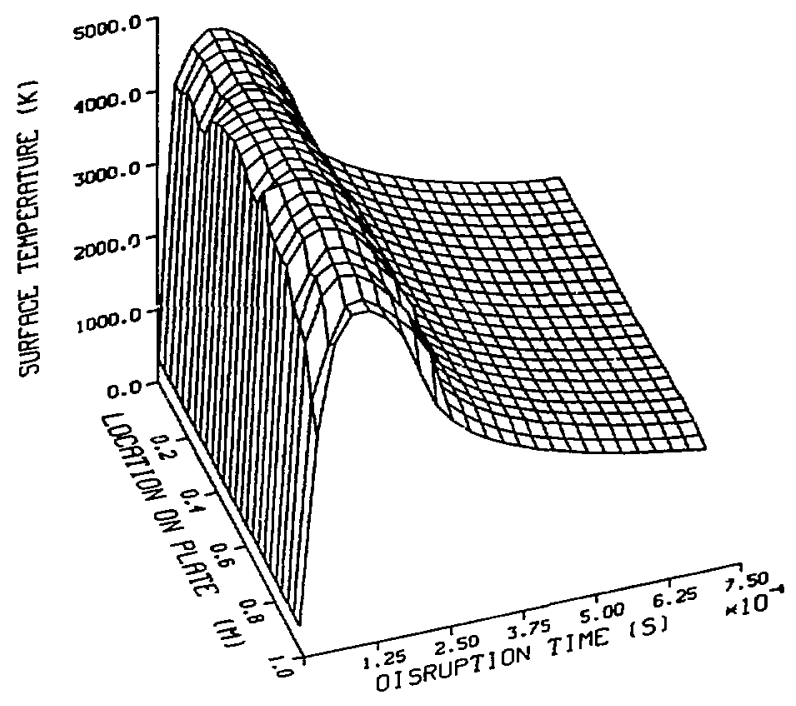

Figure 5.32: Disruption effects of $0.1 \mathrm{~ms}$ square pulse on outer divertor plate for graphite divertor material showing distribution of surface temperature. 
The first point to notice is the more gradual increase to maximum temperature for the square pulse. Here, the material is more able to respond to the lower power density through a limited amount of heat conduction, prior to the start of significant vaporization. In fact, the maximum temperature is not even reached until just before the end of the pulse. Therefore, the maximum vaporization rate will be lower, but the total amount of material vaporized may be higher. This point will be discussed with the next set of figures. The second feature of Figures 5.30, 5.31, and 5.32 to observe is the longer time scale for the square pulse, compared to the baseline case illustrated in Figures 5.4, 5.5, and 5.6. To illustrate the cooling process more fully, the time scale is approximately twice as long. Although for each plate, the maximum temperatures are 300 to 400 degrees lower for the square pulse, the average temperatures for this case are higher than for the DSTAR pulse shape. For the baseline case, the temperature abruptly reaches its maximum value and then continually decreases during the remainder of the pulse. Since the temperature profiles drive the vaporization, the corresponding ablation effects are expected to be similar to these temperature distributions. Figures $5.33,5.34$, and 5.29 , show that this prediction is true. The vaporization rate does not reach a maximum until neas the end of the disruption for the square pulse case. In fact, the figures show that significant vaporization occurs for almost $0.1 \mathrm{~ms}$ after the termination of the pulse. For the baseline case, no vaporization occurs after the pulse. The significant differences between the baseline and square pulse cases are summarized 


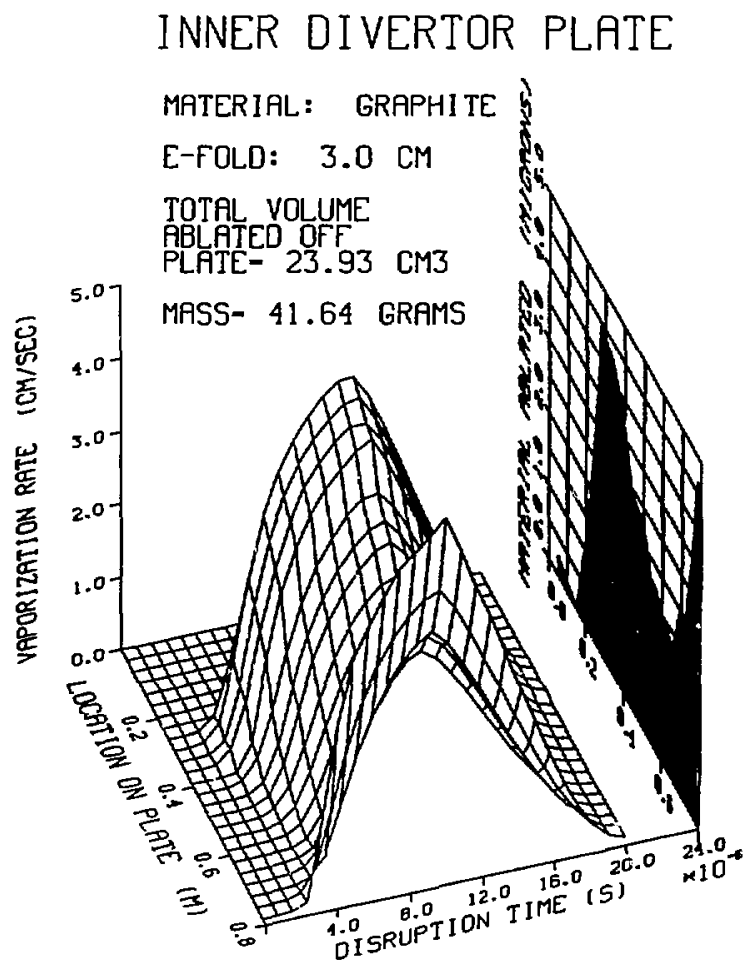

Figure 5.33: Disruption effects of $0.1 \mathrm{~ms}$ square pulse on inner divertor plate for graphite divertor material showing distribution of vaporization rate and ablation depth. 


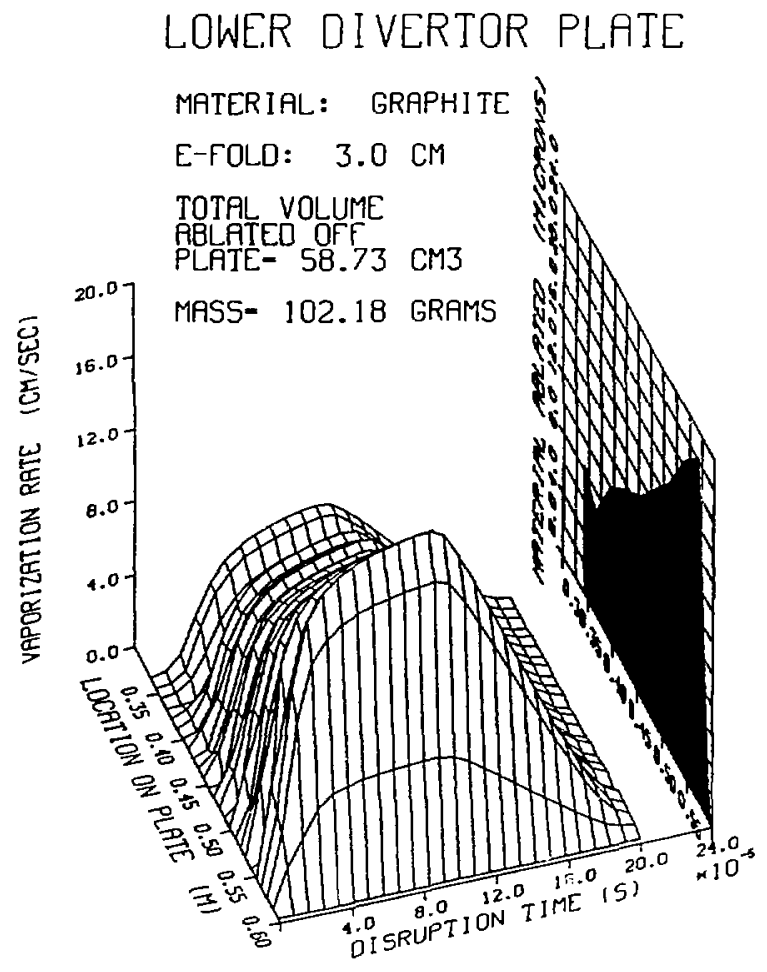

Figure 5.34: Disruption effects of $0.1 \mathrm{~ms}$ square pulse on lower divertor plate for graphite divertor material showing distribution of vaporization rate and ablation depth. 


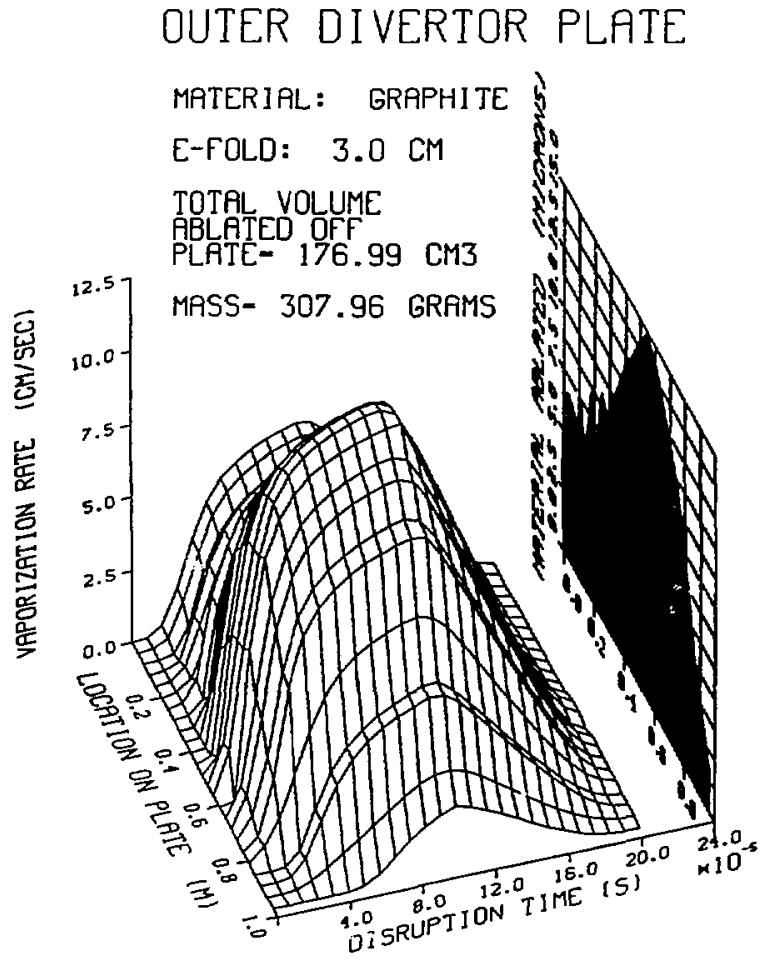

Figure 5.35: Disruption effects of $0.1 \mathrm{~ms}$ square pulse on outer divertor plate for graphite divertor material showing distribution of vaporization rate and ablation depth. 
in Table 5.5.

\subsection{Characteristic Radial Decay Length}

As discussed in Section 2.4, the disruption power flux in the scrape-off layer is assumed to decrease exponentially in distance outward from the edge of the plasma. The characteristic radial decay constant, $\lambda$, determines the relative rate of this exponential decay. An explicit metho? for calculating this constant was summarized in Section 2.4. Since this method has some uncertainty, investigating a range of possible values for $\lambda$ merits consideration. The subject of this section is the evaluation of the difference in thermal effects due to varying this parameter. Since the baseline case of this thesis has assumed a value of 3.0 $\mathrm{cm}$, a range of magnitudes from 1.0 to $4.5 \mathrm{~cm}$ was investigated for this decay constant. To place this range of values in a better perspective, it should be noted that the steady-state analysis performed for TIBER II assumed a 1.0 -cm value for the characteristic radial decay length [5.2].

Selected thermal response parameters are presented in Figures 5.36-5.41. Figure 5.36 depicts the maximum power density present on each plate as a function of the decay constant. As can be seen in this figure, the maximum power density on the inner and lower plates decreases as the decay length increases, while for the outer plate, the value remains fairly constant. The decreasing maximum power densities on the inner and lower plates result from a spreading of the particle flux through an increased $\lambda$ and the spatial variation of the 
Table 5.5: Comparison of ablation effects on graphite divertor plates due to the DSTAR and square pulse shapes for a 0.1 ms disruption.

\begin{tabular}{|c|c|c|c|}
\hline \multirow[b]{2}{*}{ Plate } & \multirow[b]{2}{*}{ Ablation Effect } & \multicolumn{2}{|c|}{ Disruption Pulse Shape } \\
\hline & & DSTAR & Square \\
\hline \multirow[t]{7}{*}{ Inner } & Maximum Power Density (MW $/ \mathrm{m}^{2}$ ) & $3.7 \times 10^{4}$ & $1.9 \times 10^{4}$ \\
\hline & Maximum Temperature (K) & 4950 & 4650 \\
\hline & Maximum Vaporization Rate $(\mathrm{cm} / \mathrm{s})$ & 14.8 & 4.9 \\
\hline & Maximum Ablation Depth (microns) & 3.4 & 4.7 \\
\hline & Peaking Factor for Ablation Depth & 2.0 & 2.0 \\
\hline & Volume Ablated off Plate $\left(\mathrm{cm}^{3}\right)$ & 18.3 & 23.9 \\
\hline & Mass Ablated off Plate $(\mathrm{g})$ & 31.8 & 41.6 \\
\hline \multirow[t]{7}{*}{ Lower } & Maximum Power Density (MW/m²) & $1.0 \times 10^{5}$ & $2.5 \times 10^{4}$ \\
\hline & Maximum Temperature (K) & 5400 & 5000 \\
\hline & Maximum Vaporization Rate $(\mathrm{cm} / \mathrm{s})$ & 14.8 & 17.6 \\
\hline & Maximum Ablation Depth (microns) & 15.0 & 22.3 \\
\hline & Peaking Factor for Ablation Depth & 1.6 & 1.6 \\
\hline & Volume Ablated off Plate $\left(\mathrm{cm}^{3}\right)$ & 39.8 & 58.7 \\
\hline & Mass Ablated off Plate (g) & 69.2 & 102 \\
\hline \multirow[t]{7}{*}{ Outer } & Maximum Power Density $\left(\mathrm{MW} / \mathrm{m}^{2}\right)$ & $7.3 \times 10^{4}$ & $9.3 \times 10^{3}$ \\
\hline & Maximum Temperature (K) & 5270 & 4900 \\
\hline & Maximum Vaporization Rate $(\mathrm{cm} / \mathrm{s})$ & 14.8 & 12.4 \\
\hline & Maximum Ablation Depth (microns) & 10.0 & 15.0 \\
\hline & Peaking Factor for Ablation Depth & 1.6 & 1.5 \\
\hline & Volume Ablated off Plate $\left(\mathrm{cm}^{3}\right)$ & 121.6 & 174 \\
\hline & Mass Ablated off Plate (g) & 211.5 & 302 \\
\hline \multirow[t]{2}{*}{ ALL } & Volume Ablated $\left(\mathrm{cm}^{3}\right)$ & 361 & 513 \\
\hline & Mass Ablated (g) & 625 & 893 \\
\hline
\end{tabular}




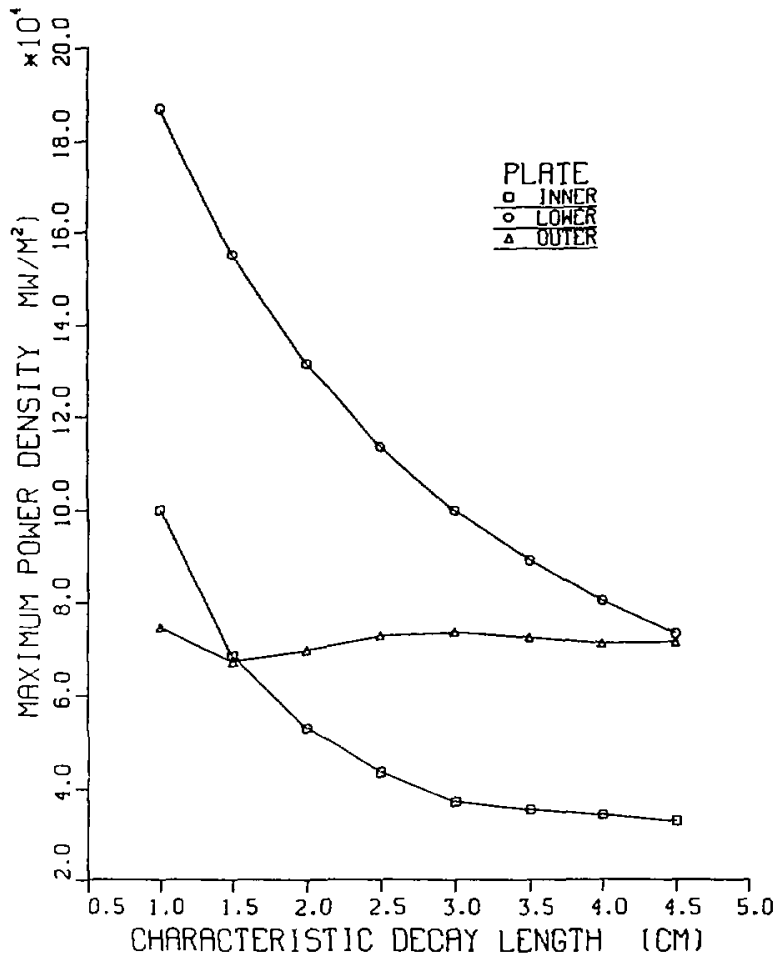

Figure 5.36: Maximum disruption power density on each graphite divertor plate as a function of characteristic radial decay length. 
magnetic flux surfaces as they intersect each plate. This observation suggests that the more diffusive the particle transport is in the SOL, the lower the power densities will be on the inner and lower divertor plates during a disruption. This inference is drawn from the fact that the decay length is proportional to the radial thermal diffusivity according to Equation 2.9 . In contrast, the outer plate appears not to be affected by a change in the diffusivity of the disruption. This lack of change can be best explained by the outer plate's spatial configuration within the reactor. Despite the power being spread over more area of the plate as the decay constant is increased, the magnetic flux surfaces are oriented in such a way to counter this decrease proportionately. Hence, the outer plate has a constant maximum power density.

Although the energy distribution changes with a change in $\lambda$, the total energy of the disruption should be conserved. Figure 5.37 indicates this prediction is true. The sum of the three curves in this figure remains fairly constant over the range evaluated. Higher values for the decay constant would more than likely result in a departure from the form of the curves presented here, since larger amounts of e:rergy would be deposited on surfaces beyond the edges of the divertor plates. This lack of conservation would result from particles reaching flux surfaces that intersect other regions of the first wall. The other interesting feature of this set of curves is how the three plates share the total energy available. For the steady-state decay constant of $1.0-\mathrm{cm}$, the lower and outer plates receive equal amounts of energy. Increasing $\lambda$ results in sweeping 


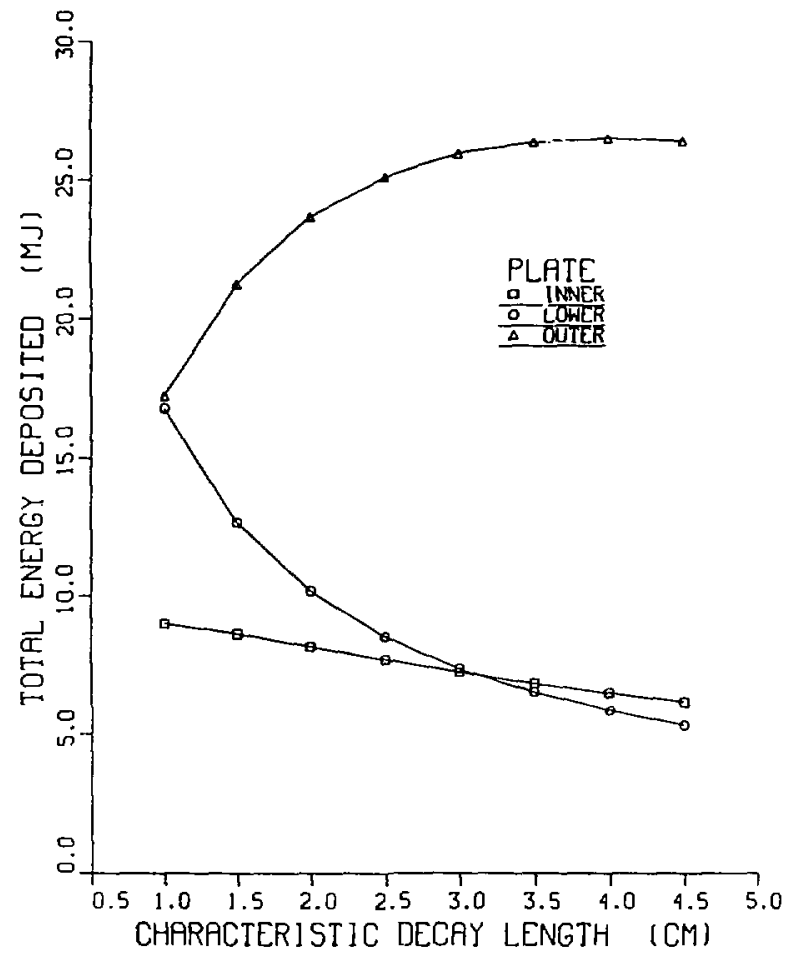

Figure 5.37: Total energy deposited on each graphite divertor plate as a function of characteristic radial decay length. 
the energy from the lower plate to the outer plate. Although such a movement in the power distribution is expected, the particular form of this change is not intuitively obvious. Again, the partial dependence of the magnetic flux surfaces combines with the changing decay length to determine this change in the tntal energy distribution.

Figures 5.38-5.40 must be considered to fully appreciate the significance of varying the magnitude of the characteristic decay constant. Specifically, the impact of this variation on divertor design for disruption conditions can be inferred from these figures. The maximum surface temperature, maximum vaporization rate, and maximum vaporization depth as a function of the decay length are presented in Figures 5.38, 5.39, and 5.40, respectively. Each figure has the same relative form. As .eviously mentioned, this similarity is due to the way in which the temperature and vaporization rate are mathematically coupled in the formulation of the moving boundary problem. Upon inspection, Figure 5.40 implies that the inner and lower plates benefit from a larger decay length in the event of a disruption. In other words, the maximum amount of material ablated from the inner and lower plates decreases as $\lambda$ increases. However, for the outer plate, no significant differences are apparent.

Figure 5.41 in this section depicts the total volume ablated as a function of the decay constant. Despite the large decrease in the local ablation from the inner and lower plates shown in Figures 5.39 and 5.40, the global volume ablated from all three plates decreased only slightly, as seen in Figure 5.41. This 


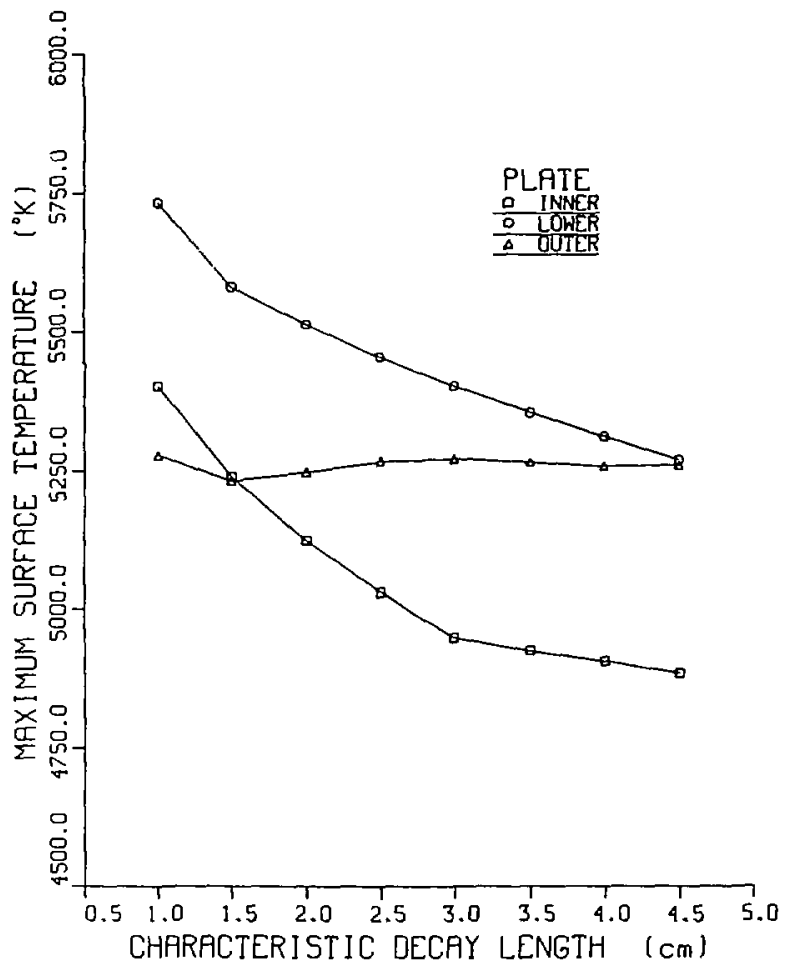

Figure 5.38: Maximum surface temperature on each graphite divertor plate as a function of characteristic radial decay length. 


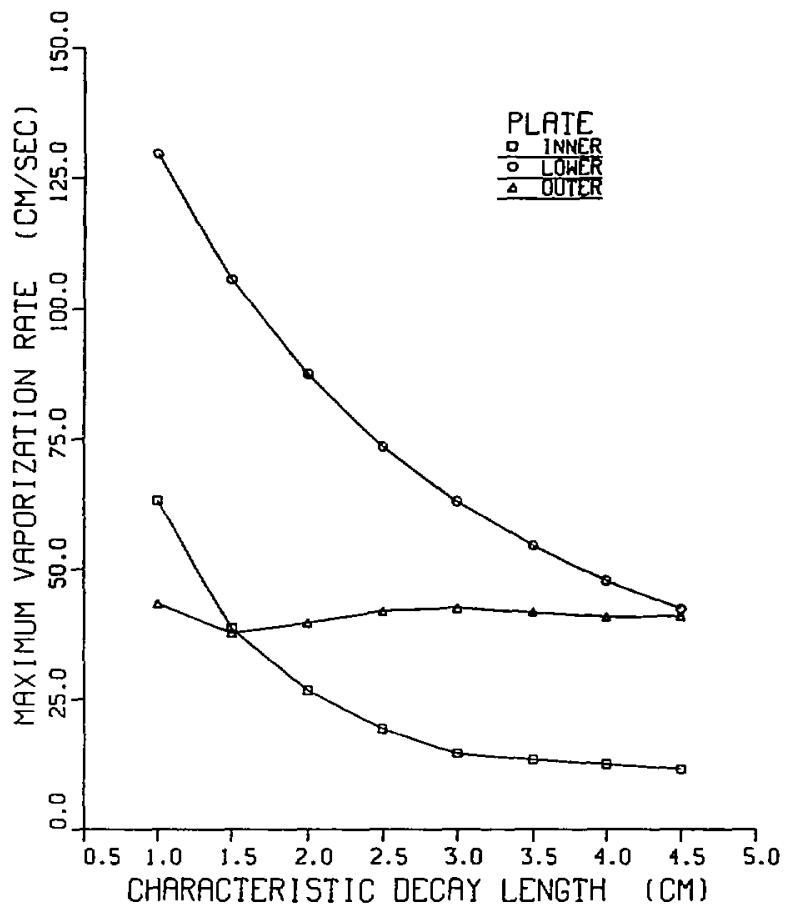

Figure 5.39: Maximum vaporization rate on each graphite divertor plate as a function of characteristic radial decay length. 


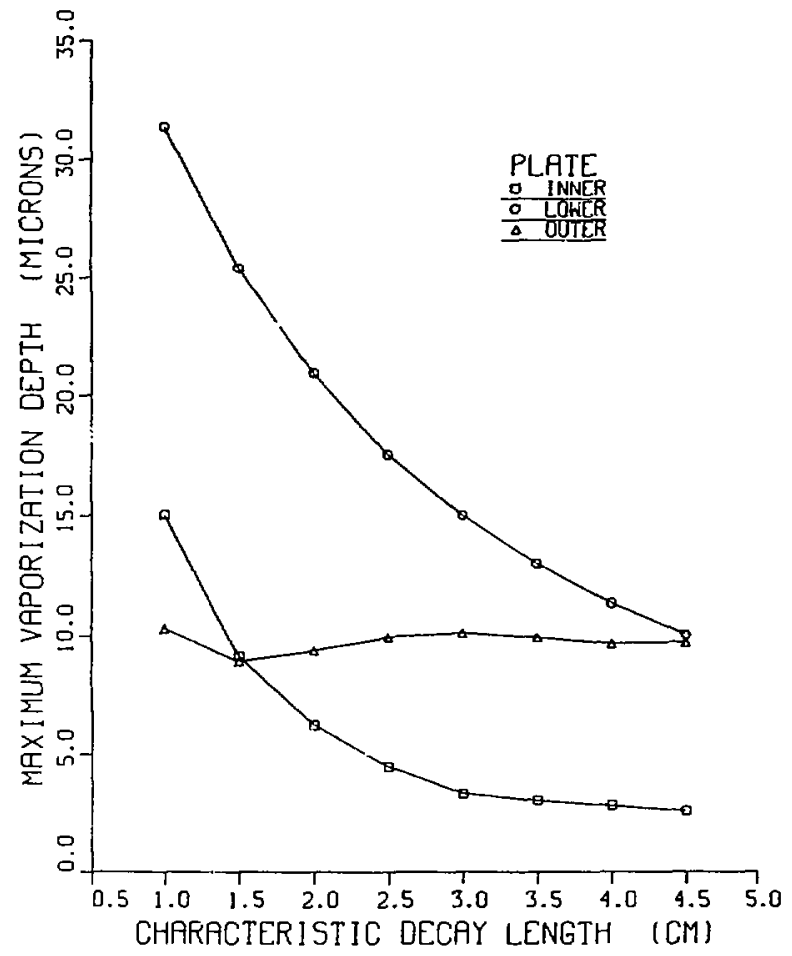

Figure 5.40: Maximum vaporization depth on each graphite divertor plate as a function of characteristic radial decay length. 
discrepancy can be attributed to the radial dependence of the total affected surface area and the higher ablation occurring further out in radial distance on each plate. Since the locations of the highest vaporization rates are moving radially outward on each plate, the surface area most affected is increasing as the square of the distance from the center of the reactor. 


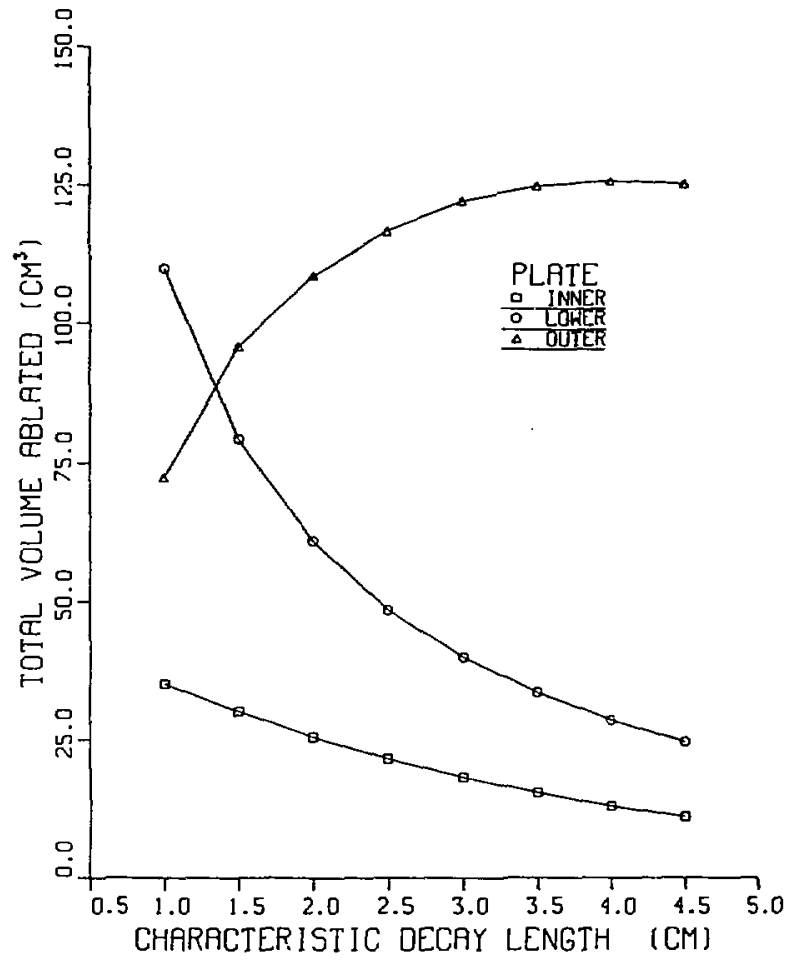

Figure 5.41: Total volume ablated on each graphite divertor plate as a function of characteristic radial decay length. 


\subsection{References for Chapter 5}

5.1 J.R. Haines, "Proceedings of the Japan-US Q-70 Exchange on FER and TIBER Design," Lawrence Livermore National Laboratory (24 February 1987).

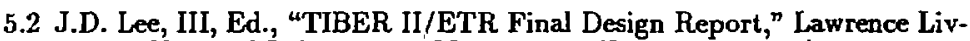
ermore National Laboratory, UCID-21150, (September 1987). 


\section{Chapter 6}

\section{Conclusions and \\ Recommendations for Further Investigation}

\subsection{Conclusions}

The main objective of this dissertation was to predict the thermal response of a tokamak divertor design through the extension of previous disruption modeling. As a result of this effort, the hybrid computational model TADDPAK evolved. Through its use, useful conclusions concerning specific divertor design issues can be reached. However, as with any theoretical analysis, the conclusions drawn from this parametric study are only as good as the assumptions and models upon which they are based. Since the understanding of the physics of plasma disruptions is still evolving, only reasonable assumptions, based on the experience gained from existing tokamak experiments, can be made. The trive test of these assumptions will only occur through experimental validation. 
Regardless of these caveats, conclusions made from this modeling approach are very useful since they provide important information for initial divertor design and raise important theoretical questions.

The following specific conclusions can be drawn from this parametric study:

1) The magnetic flux surfaces present in the scrape-off layer of a plasma equilibrium play a significant role in determining the spatial dependence of the thermal response on a tokamak divertor during the thermal quench of a plasma disruption.

2) 'The lower plate of the divertor system within the TIBER $I 1$ configuration receives the highest power and energy densities, surface temperature, and local vaporization.

3) The outer plate, mainly due to its larger surface area, has the most energy deposited and volume ablated during the thermal quench of a disruption.

4) The amount of vaporization that occurs during a disruption is very sensitive to the maximum temperature reached on the surface of the material. In addition, the temperature dependence of the vapor pressure is critical in determining the resulting vaporization.

5) Maximum surface temperatures reached during the disruption are signifcantly higher for tungsten than for graphite and beryllium. 
6) Beryllium divertor plates suffered the largest amount of ablation, compared to plates composcil: of graphite or tungsten.

7) Of the three materials, tungsten divertor plates had the least volume ablated per disruption.

8) The best material choice for the divertor plates under a disruption scenario and based on maximum allowable thicknesses is graphite.

9) The highest peaking factor for ablation was 6.7. It occurred on an inner divertor plate of tungsten for the baseline case of a $1.0-\mathrm{ms}$ thermal quench and a characteristic radial decay length of $3.0 \mathrm{~cm}$. Graphite and beryllium had corresponding peaking factors of 2.0 and 2.1 , respectively.

10) Dramatic changes in the ablation effects on the divertor plates occur over the current range of interest for the disruption pulse length. These changes are the most pronounced in Figure 5.24. Such wide differences emphasizes how accuracy in predicting the duration of a disruption can influence designing components against disruptions.

11) Peaking factors for all three plates approach a minimurn value as the pulse length of the disruption decreases to zero.

12) Pulse shape can have a significant impact on the total ablation siffered by a divertor plate. For the time scale and stored energy of this study, the square pulse shape results in 30 to $40 \%$ higher ablation effects than 
the DSTAR pulse shape, which is exponential in form. This difference suggests that past analysis, which assumed a square pulse shape, may have been somewhat conservative.

13) The magnitude of the characteristic radial decay length has a significant impact on the spatial dependence of ablation effects on the three divertor plates. Over the range of values investigated, the ablation effects on the outer plate remained fairly constant. However, for the inner and lower plates, ablation effects decreased significantly when the decay length was increased. Larger values for the decay constant soften the impact a disruption has on the divertor. However, the impact may appear on surfaces other than the divertor.

\subsection{Future Work}

Until scientists and engineers have successfully built and operated an engineering test reactor, there will be many unanswered questions concerning the phenomenon of disruptions. The work of this thesis touches upon one of the areas still under investigation. Some of the key issues addressed in this thesis that still require investigation are as follows:

1) More experimental data are needed on high-temperature vapor pressures for materials relevant to divertor design. The validity of these data impacts significantly the accuracy of vaporization modeling. Especially relevant to this modeling are the critical temperatures of candidate materials. 
Table 6.1: Summary of ablation effects on divertor plates of graphite, tungsten, and beryllium subjected to $0.1-\mathrm{ms}$ disruption.

\begin{tabular}{||l|l|r|r|r||}
\hline \multirow{3}{*}{ Plate } & Ablation Effect & \multicolumn{3}{|c|}{ Material } \\
\cline { 2 - 5 } & Graphite & Tungsten & Beryllium \\
\hline Inner & Maximum Temperature (K) & 4950 & 6202 & 3564 \\
& Maximum Vaporization Rate (cm/s) & 14.8 & 0.63 & 21.3 \\
& Maximum Ablation Depth (microns) & 3.4 & 0.11 & 5.1 \\
& Peaking Factor for Ablation Depth & 2.0 & 6.7 & 2.1 \\
& Volume Ablated off Plate (cm ${ }^{3}$ ) & 18.3 & 0.18 & 26.3 \\
& Mass Ablated off Plate (g) & 31.8 & 3.4 & 48.7 \\
\hline Lower & Maximum Temperature (K) & 5400 & 8650 & 4251 \\
& Maximum Vaporization Rate (cm/s) & 14.8 & 40.2 & 101.5 \\
& Maximum Ablation Depth (microns) & 15.0 & 9.1 & 24.4 \\
& Peaking Factor for Ablation Depth & 1.6 & 2.1 & 1.6 \\
& Volume Ablated off Plate (cm $\left.{ }^{3}\right)$ & 39.8 & 18.8 & 64.5 \\
& Mass Ablated off Plate (g) & 69.2 & 363.4 & 119.4 \\
\hline Outer & Maximum Temperature (K) & 5270 & 8170 & 4052 \\
& Maximum Vaporization Rate (cm/s) & 14.8 & 21.8 & 68.2 \\
& Maximum Ablation Depth (microns) & 10.0 & 4.8 & 16.4 \\
& Peaking Factor for Ablation Depth & 1.6 & 2.4 & 1.6 \\
& Volume Ablated off Plate (cm $\left.{ }^{3}\right)$ & 121.6 & 39.8 & 194.8 \\
& Mass Ablated off Plate (g) & 211.5 & 769.1 & 360.4 \\
\hline ALL & Volume Ablated (cm ${ }^{3}$ ) & 361 & 118 & 571 \\
& Mass Ablated (g) & 625 & 2272 & 1057 \\
\hline
\end{tabular}


2) Future studies need to consider the effects of vapor shielding on a twodimensional level and account for the backscattering of particles that would be present at the divertor surface. Although not used for this study, TADDPAK does have a simple vapor shielding model that could be employed.

3) The existence of stable magnetic surfaces in the scrape-off layer during the thermal quench of a plasma disruption needs to be better substantiated by experiment.

4) Other materials for the divertor plates should be evaluated. Possible candidates include carbon-carbon composites and silicon carbides.

5) TADDPAK should be used as a design tool in actually determining the best spatial configuration for the divertor plates under a disruption scenario.

6) The validity of the assumption of melt layer stability in a magnetic field requires further investigation. Any lack of stability in this layer would greatly increase the damage incurred during a disruption.

7) More accurate data are needed on the disruption source term, including, data on pulse length, pulse shape, and energy partitioning. Only through the collection and analysis of data from experiments, will the complex phenomenon of disruptions be better understood. 


\section{Appendix A}

\section{TADDPAK User Information}

TADDPAK is availab ${ }^{\circ}$ on the Department of Energy (DOE), Office of Fusion Energy's (OFE's) Computer Center at Lawrence Livermore National Laboratory: the National Magnetic Fusion Energy Computer Center (NMFECC). The FORTRAN source code is named TADDPAK. This source code and all input files are stored in the NMFECC's file storage system, which can be accessed with the FILEM command:

FILEM READ .30201 pak pak.lb

The file pak.lb is a library file containing the files defined in Table A.1. These files are required to execute the code successfully. The source code runs on all Cray-machines (B, C, D, E). When compiling the code, use the CIVIC compiler from within the line editor TRIXGL. The RUN command from within this editor will compile, load, and create the executable XTADDPAK. Executing XTADDPAK will generate an output graphics file with the name F3?PAK0X, where? is a letter from $\mathrm{A}-\mathrm{Z}$. 
Table A.1: Descriptions of files found in PAK.LB

\begin{tabular}{||l|l||}
\hline File Name & Description \\
\hline BEPAKIN & Beryllium material properties \\
BPDAT & Poloidal magnetic surface coordinates and magnitudes \\
CPAKIN & Graphite material properties \\
DICTION & Input file variable definitions \\
DIVPTS & Divertor coordinates in poloidal cross section \\
POWERPAK & Input disruption power profile \\
TADDPAK & Source code \\
WPAKIN & Tungsten material properties \\
\hline
\end{tabular}




\section{Appendix B}

\section{Preliminary Results for US ITER}

TADDPAK has been applied to the current US ITER (International Thermonuclear Experimental Reactor) $4.04 \mathrm{~m}$ baseline. Baseline information is available on the NMFECC System. Access file ITERINFO in directory .ITER under user-number 30342 for more information. A stored thermal energy of approximately $338 \mathrm{MJ}$ is the disruption energy available during the thermal quench. An appropriately scaled disruption power profile, generated by DSTAR, was used as the initial input for the l'TER calculations. Results for both tungsten and graphite as the divertor plate material are presented in Figures 1-6. From Figures $4-5$ for tungsten, maximum ablated depths of $\approx 34 \mu \mathrm{m}$ per disruption are obtained, with $\approx 10 \mathrm{~kg}$ of material evolving from the divertor surfaces; peaking factors of $\approx 2.5$ in power energy deposition and $\approx 8$ in ablated depth are seen. 

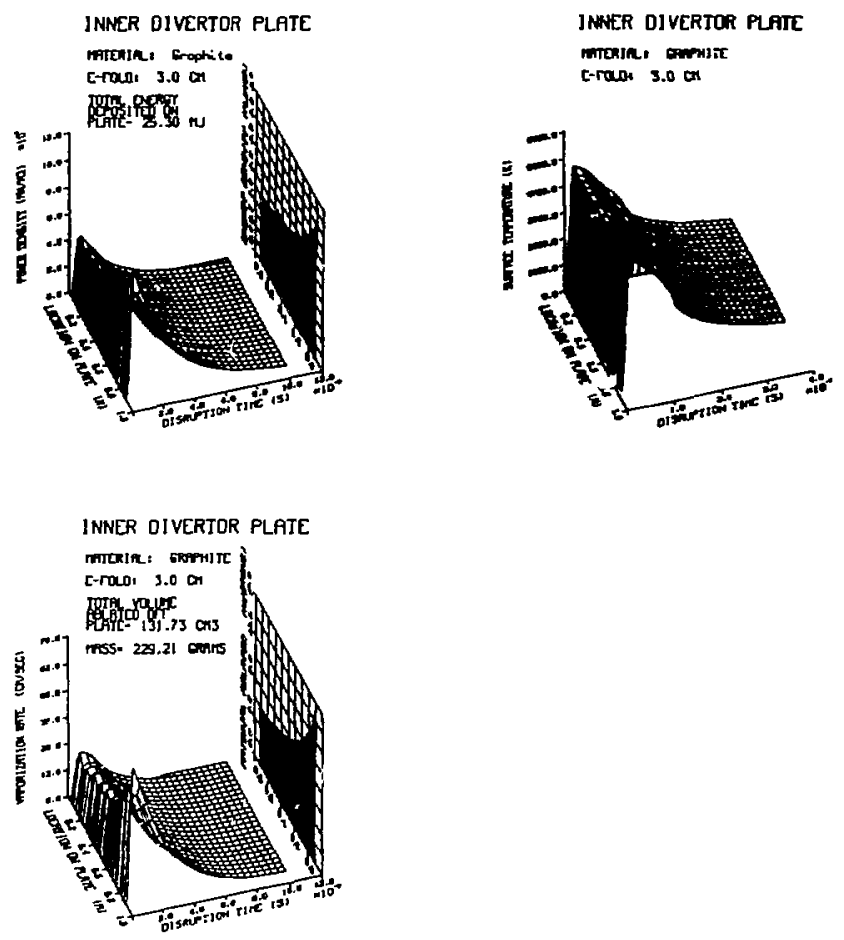

Figure B.1: Disruption conditions on the inner divertor plate for graphite divertor material showing distributions of power/energy densities, surface temperature, and vaporization rate/ablation depth. 

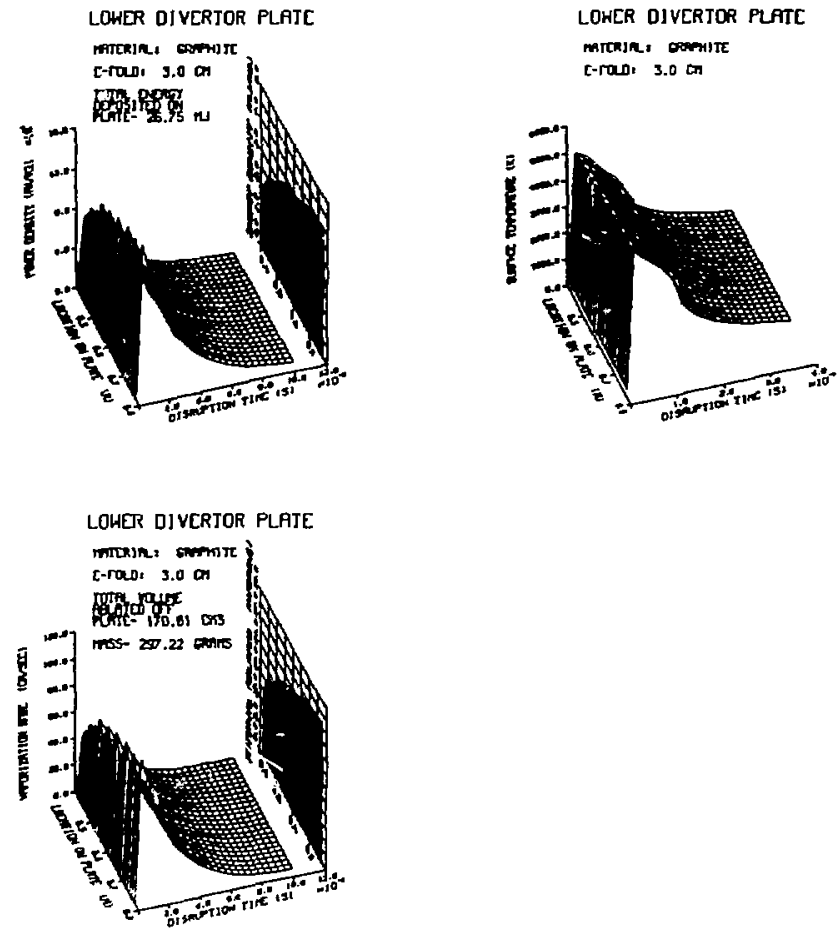

Figure B.2: Disruption conditions on the lower divertor plate for graphite divertor material showing distributions of power/energy densities, surface temperature, and raporization rate/ablation depth. 

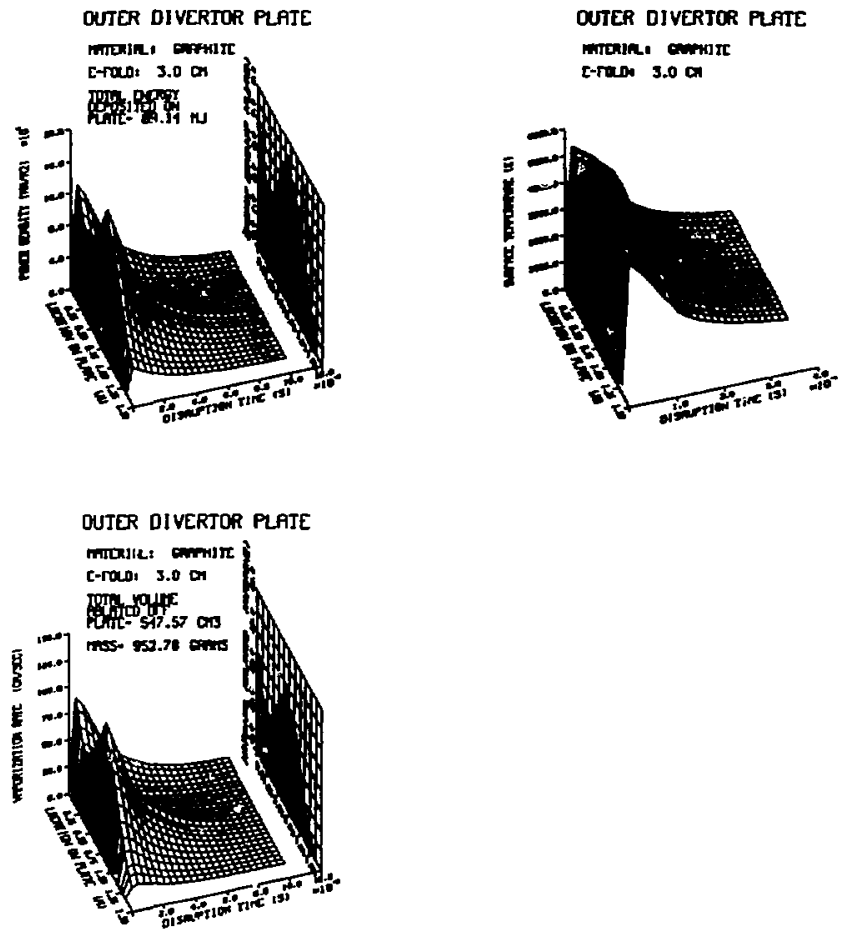

Figure B.3: Disruption conditions on the custer divertor plate for graphite divertor material showing distributions of power/energy densities, surface temperature, and vaporization rate/ablation depth. 

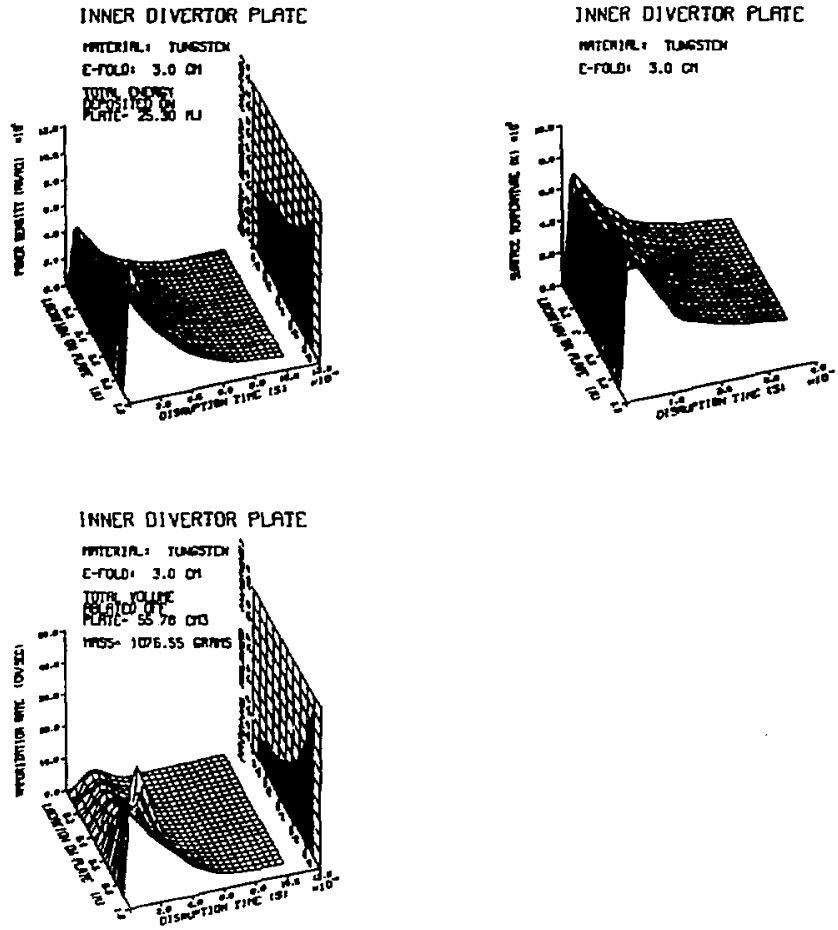

Figure B.4: Disruption conditions on the inner divertor plate for tungsten divertor material showing distributions of power/energy densities, surface temperature, and vaporization rate/ablation depth. 

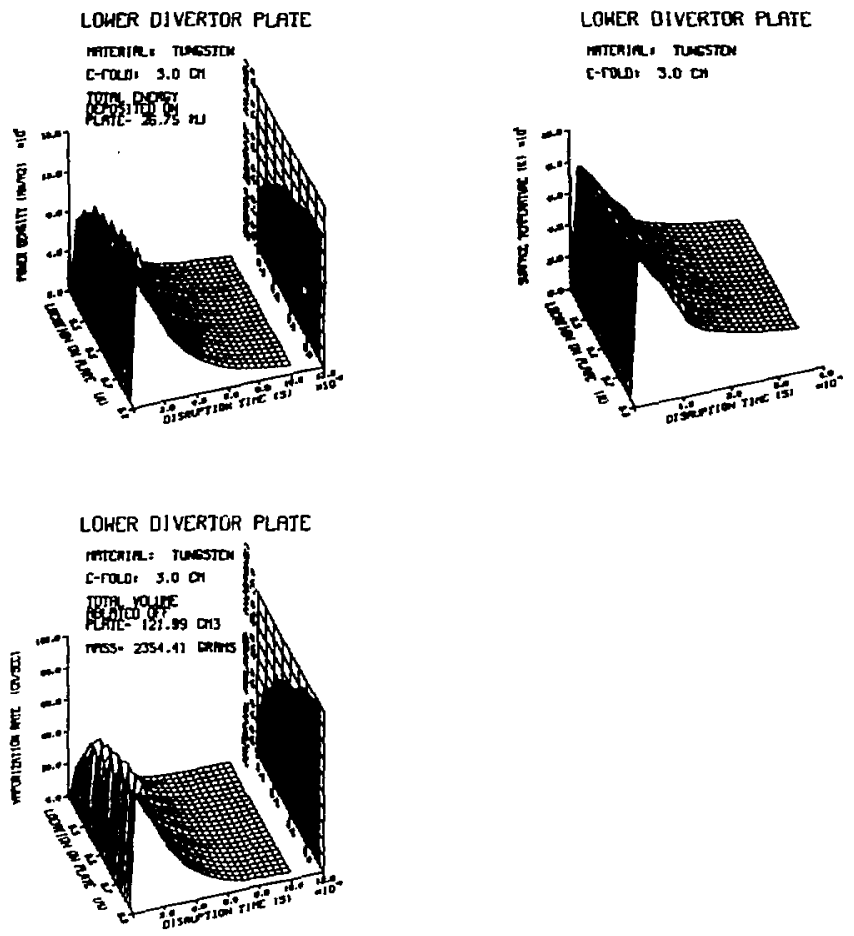

Figure B.5: Disruption conditions on the lower divertor plate for tungsten divertor material showing distributions of power/energy densities, surface temperature, and vaporization rate/ablation depth. 

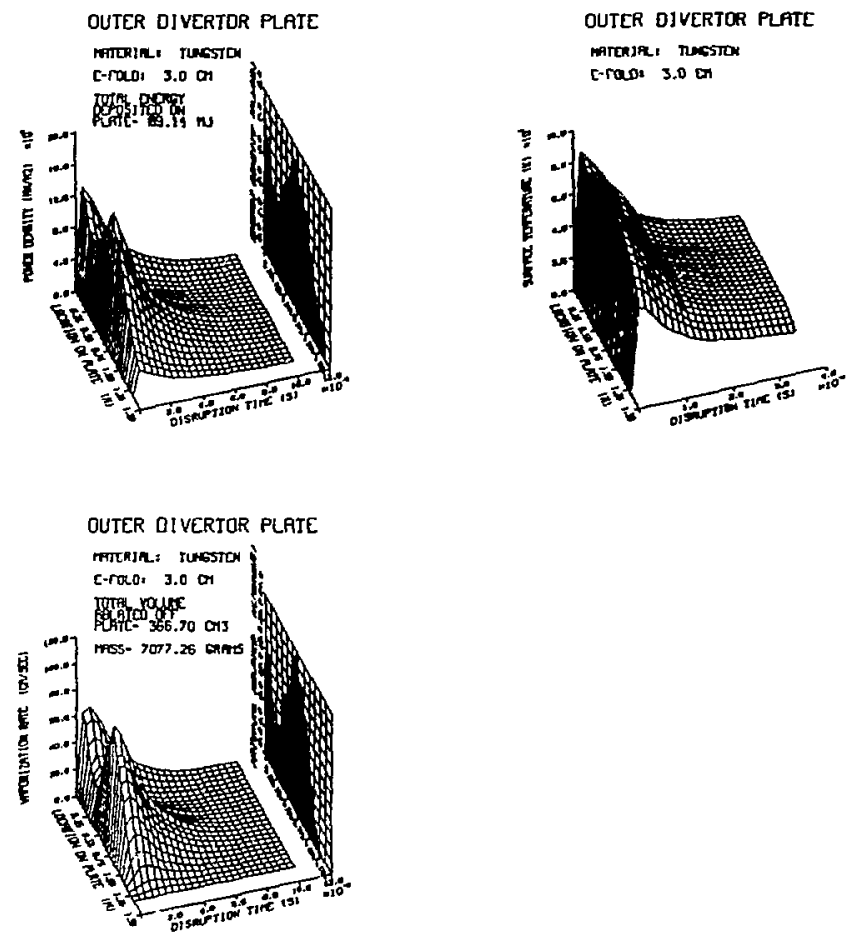

Figure B.6: Disruption conditions on the outer divertor plate for tungsten divertor material showing distributions of power/energy densities, suriace ternperature, and vaporization rate/ablation depth. 